\title{
Dietary cholesterol effects on learning, memory and amyloid beta: Learning and memory effects on brain cholesterol and sulfatide levels
}

\author{
Deya S. Darwish \\ West Virginia University
}

Follow this and additional works at: https://researchrepository.wvu.edu/etd

\section{Recommended Citation}

Darwish, Deya S., "Dietary cholesterol effects on learning, memory and amyloid beta: Learning and memory effects on brain cholesterol and sulfatide levels" (2010). Graduate Theses, Dissertations, and Problem Reports. 3252.

https://researchrepository.wvu.edu/etd/3252

This Dissertation is protected by copyright and/or related rights. It has been brought to you by the The Research Repository @ WVU with permission from the rights-holder(s). You are free to use this Dissertation in any way that is permitted by the copyright and related rights legislation that applies to your use. For other uses you must obtain permission from the rights-holder(s) directly, unless additional rights are indicated by a Creative Commons license in the record and/ or on the work itself. This Dissertation has been accepted for inclusion in WVU Graduate Theses, Dissertations, and Problem Reports collection by an authorized administrator of The Research Repository @ WVU.

For more information, please contact researchrepository@mail.wvu.edu. 


\title{
Dietary Cholesterol Effects on Learning, Memory and Amyloid Beta: Learning and Memory Effects on Brain Cholesterol and Sulfatide Levels
}

\author{
Deya S. Darwish \\ Dissertation submitted to the \\ School of Medicine \\ at West Virginia University \\ in partial fulfillment of the requirements \\ for the degree of \\ Doctor of Philosophy \\ in \\ Neuroscience
}

\author{
Bernard G. Schreurs, Ph.D., Chair \\ Albert S. Berrebi, Ph.D., Program Director \\ Gregory W. Konat, Ph.D. \\ Adrienne K. Salm, Ph.D. \\ Jason D. Huber, Ph.D. \\ Department of Neurobiology and Anatomy \\ The Center for Neuroscience \\ Morgantown, West Virginia \\ 2010
}

Keywords: learning, memory, cholesterol, sulfatide, copper, amyloid beta 


\title{
Abstract \\ Dietary Cholesterol Effects on Learning, Memory and Amyloid Beta: Learning and Memory Effects on Brain Cholesterol and Sulfatide Levels
}

\author{
Deya S. Darwish
}

Recent studies have revealed ambiguous findings on the effects of dietary cholesterol on learning. In one study by Schreurs and coworkers it was reported that a 2\% cholesterol diet had a positive effect on learning. However, it was realized that metals, specifically copper, could potentially exacerbate the aggregation of amyloid beta $(\mathrm{A} \beta)$, a protein associated with Alzheimer's disease (AD) leading to the formation of $A \beta$ plaques. In a subsequent publication by Sparks and Schreurs it was reported that a $2 \%$ cholesterol diet with the addition of $0.12 \mathrm{ppm}$ copper had a detrimental effect on learning and resulted in the formation of $A \beta$ plaques. To better understand the effects of cholesterol and copper on learning and to begin exploring cholesterol's effect on memory we conducted a series of experiments.

In a first study, we investigated the effects of dietary cholesterol and copper on learning. Rabbits fed a diet varying in cholesterol concentration $(0,0.5,1$, and $2 \%)$ with $0.12 \mathrm{ppm}$ copper added to the drinking water received Pavlovian conditioning during which levels of learning were assessed. Analysis of $A \beta$ staining, showed a significant cholesterol concentration-dependent increase in the number of $A \beta$ positive neurons in the cortex of the cholesterol-fed rabbits. Learning was significantly greater in the $2 \%$ cholesterol-fed rabbits over controls. The data suggested that dietary cholesterol may facilitate learning and memory in the absence of $\mathrm{A} \beta$ plaques.

Next, we investigated dietary cholesterol effects on memory retention. We showed that dietary cholesterol had an adverse effect on memory retention of a previously learned task. It is still debatable whether or not dietary cholesterol affects brain cholesterol levels and there are no studies investigating sulfatide levels. Our data suggest that although dietary cholesterol affects memory retention, it does not do so by directly affecting cholesterol or sulfatide levels in the brain, suggesting peripheral effects may be mediated through secondary mechanisms. On the other hand, our data show that brain levels of cholesterol and sulfatides do change as a function of learning and memory.

Finally, in light of these findings, we investigated whether the changes in brain cholesterol and sulfatide levels occurred as a result of a learning task alone and if they occurred quickly or required several months to develop as seen in our previous memory retention experiment. We found significant changes in sulfatide levels as a function of time but no changes as a function of learning in either brain cholesterol or sulfatide levels. 
This work is dedicated to my loving and supportive parents

Saker and Ghaziha 


\section{Acknowledgements}

This work could not have been done without the support and help of all the members of the Schreurs' lab. In particular I would like to thank Carrie Smith-Bell for teaching me how to conduct behavioral experiments using rabbit classical conditioning, as well as how to analyze behavior data. I would like to also thank Lauren Burhans, Desheng Wang, Roger Bell and Jimena Gonzales for assisting me in collecting data.

Dr. Berrebi thank you for serving on my committee and for the many years you were my graduate program director. Dr. Huber and Dr. Salm thank you also for taking the time to serve on my committee and your guidance and advice throughout my graduate studies.

Dr. Konat thank you for taking many days to teach me all the biochemical techniques necessary for brain cholesterol and sulfatide analysis, and thanks for the many discussions we had both scientific and life related.

Dr. Schreurs thank you for all your guidance, patience and support throughout the years. Many thanks especially for your countless hours reading, editing, and revising the many documents I have had to prepare and submit to fulfill the requirements for my graduate studies. 


\section{Table of Contents}

page

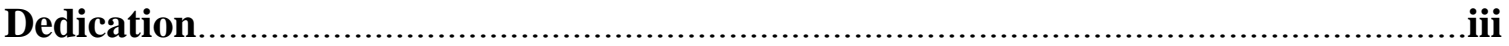

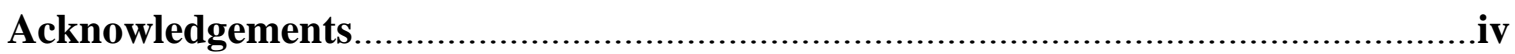

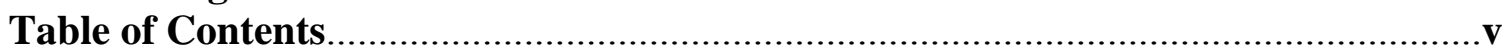

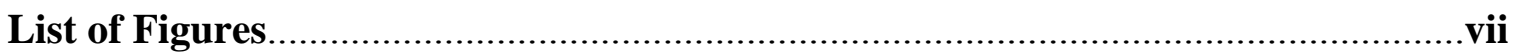

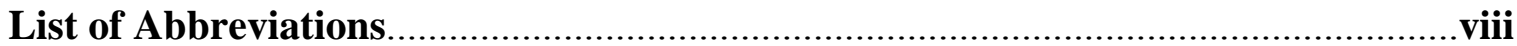

\section{Chapter 1: Introduction}

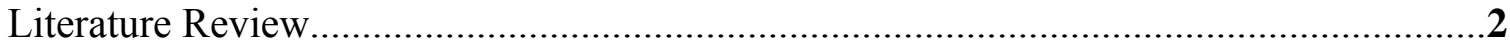

Chapter 2: Objectives and Rationale

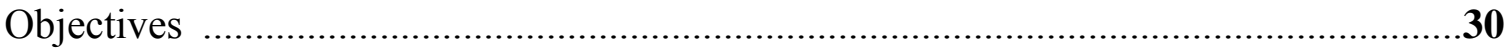

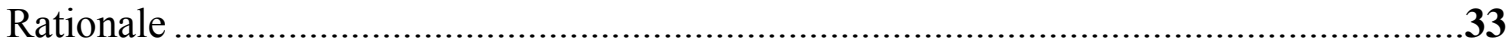

Chapter 3: Preliminary Data: Effects of dietary cholesterol on learning and memory retention

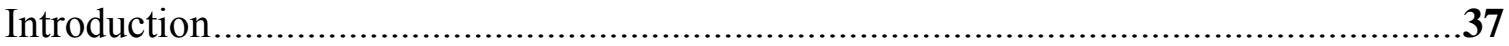

Summary

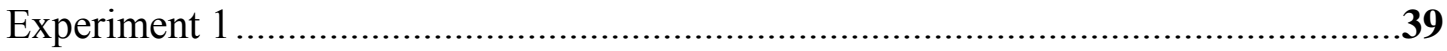

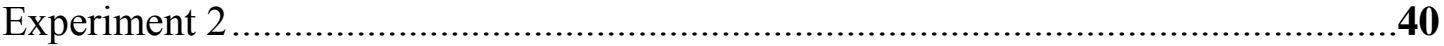

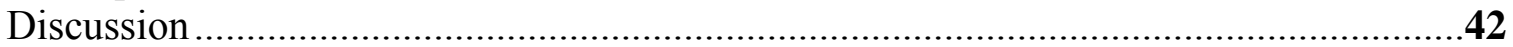

Chapter 4: Dietary cholesterol and copper effects on learning

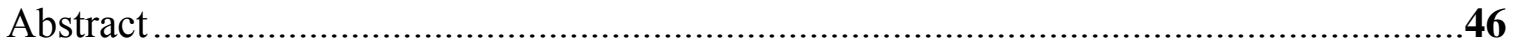

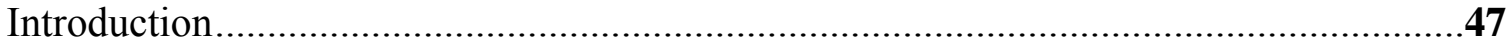

Materials and Methods...................................................................................49

Results

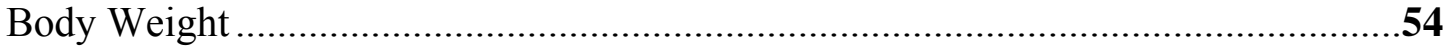

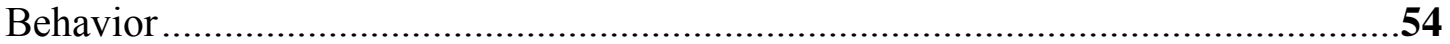

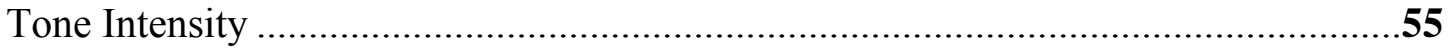

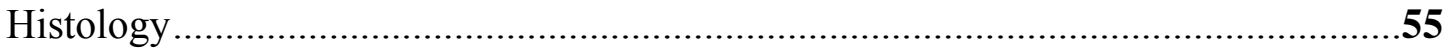

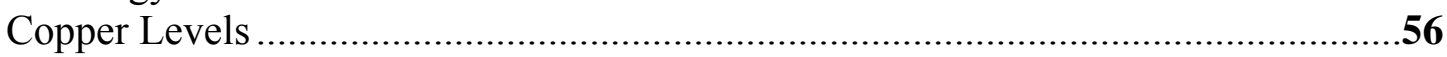

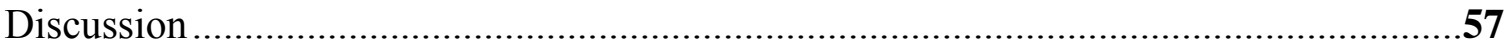


Chapter 5: Dietary cholesterol impairs memory and memory increases brain cholesterol and sulfatide levels

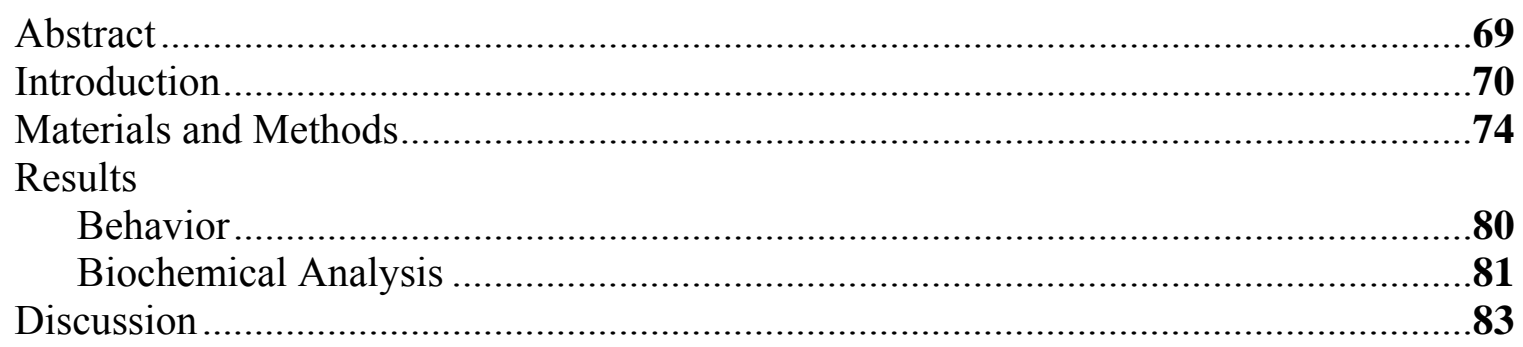

Chapter 6: Trace conditioning alone does not increase brain sulfatide and cholesterol levels

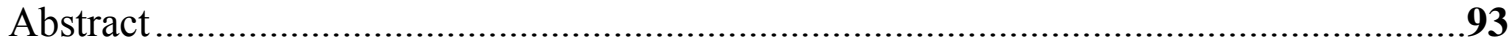

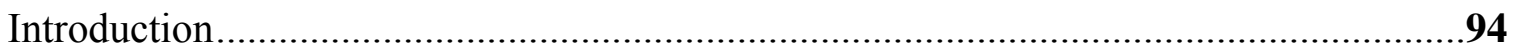

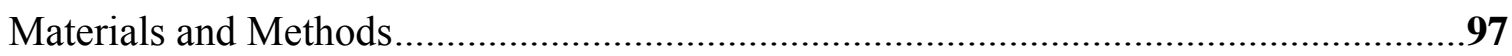

Results

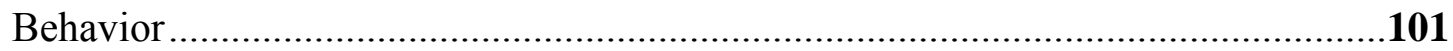

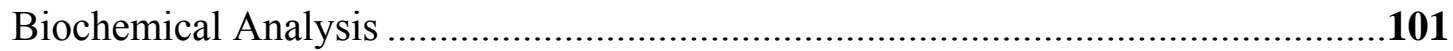

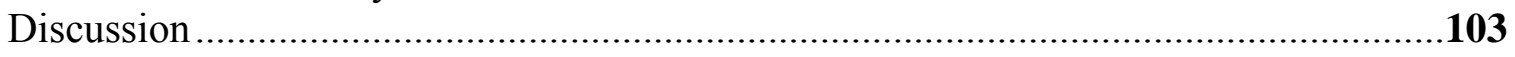

Chapter 7: General Discussion

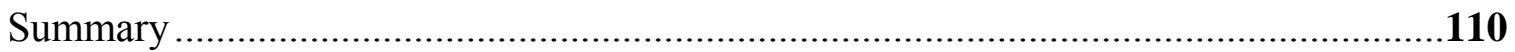

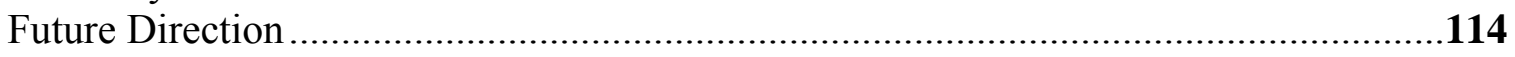




\section{Index of Figures}

\section{Chapter 1}

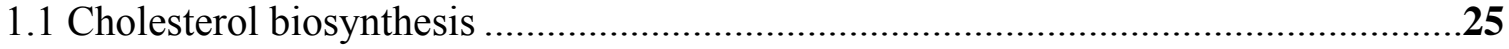

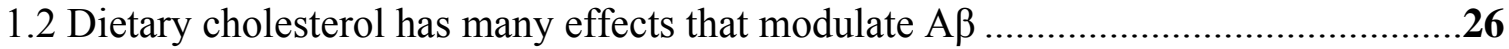

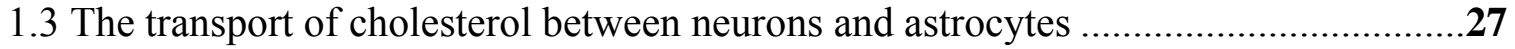

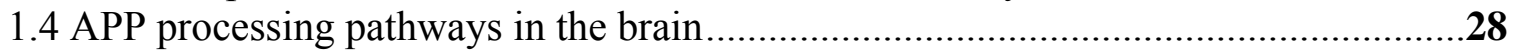

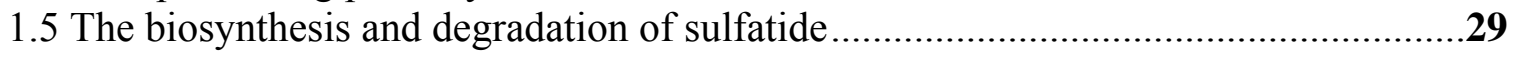

\section{Chapter 3}

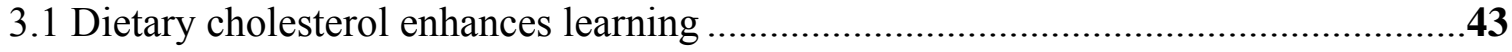

3.2 Dietary cholesterol impairs memory ................................................................44

\section{Chapter 4}

4.1 Mean body weight for rabbits

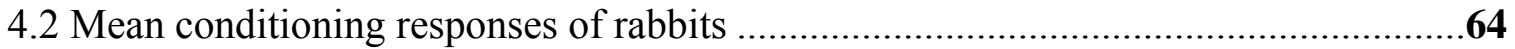

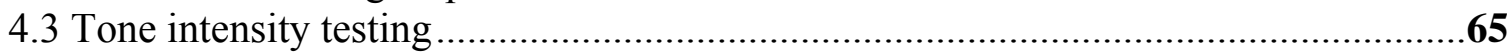

4.4 Mean number amyloid beta immunoreactive (labeled) cells ..................................66

4.5 Low power photomicrographs of representative amyloid beta

Immunoreactive (labeled) cells

\section{Chapter 5}

5.1 Mean conditioned responses in acquisition and extinction...................................88

5.2 Cholesterol and sulfatide levels as a function of diet ..........................................89

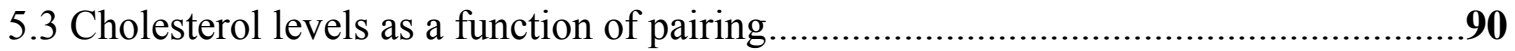

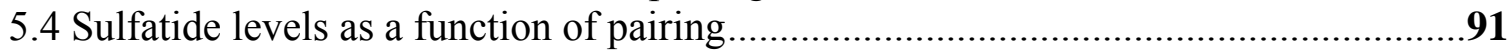

\section{Chapter 6}

6.1 Mean percent conditioned responses from trace conditioning …..........................106

6.2 Cholesterol and sulfatide levels as a function of pairing ...................................107

6.3 Sulfatide levels increase as a function of time..................................................108 


\section{List of Abbreviations}

\begin{tabular}{|c|c|}
\hline$A \beta \ldots \ldots \ldots$ & ...amyloid beta \\
\hline ABCA1.... & ... ATP-binding cassette A1 \\
\hline AD ............ & ...Alzheimer's disease \\
\hline ADAMs... & .....a disintegrin and metalloproteinase \\
\hline AICD .... & .. APP intracellular domain \\
\hline AMPA........ & $\begin{array}{c}\ldots \alpha \text {-amino-3-hydroxy-5-methyl-4- } \\
\text { isoxazolepropionic acid receptor }\end{array}$ \\
\hline ANOVA.... & $\ldots$ analysis of variance \\
\hline AP $\ldots \ldots \ldots \ldots$ & ... air puff \\
\hline Aph-1......... & ....anterior pharynx-defective \\
\hline APP..... & ....amyloid precursor protein \\
\hline ApoE. & .....apolipoprotein E \\
\hline ASA.... & ... arylsulfatase $\mathrm{A}$ \\
\hline BACE1. & $\ldots \beta$-site APP cleaving Enzyme 1 \\
\hline BBB.... & ...blood brain barrier \\
\hline CGT.. & ...ceramide galactosyltransferase \\
\hline CNS........... & ...central nervous system \\
\hline CR.............. & ... conditioned response \\
\hline CS............. & .. .conditioned stimulus \\
\hline CST............ & ....cerebroside sulfotransferase \\
\hline ER.............. & ....estrogen receptors \\
\hline HMG-CoAR. & ....3-hydroxy-3-methylglutaryl-CoA reductase \\
\hline IDE............ & ....insulin degrading enzyme \\
\hline LDLR. & ....low density lipoprotein receptor \\
\hline LRP.... & ...LDL receptor-related protein \\
\hline LXR.... & ....liver X receptor \\
\hline MAPK.. & ....Mitogen-activated protein kinase \\
\hline MLD........ & .... Metachromatic Leukodystrophy \\
\hline NCT........... & ... nicastrin \\
\hline NMDA..... & $\ldots . N$-methyl $D$-aspartate \\
\hline NMR..... & .... nictitating membrane response \\
\hline PAPS .......... & ....3'-phosphoadenosine-5'-phosphosulfate \\
\hline PD............ & .... Parkinson's disease \\
\hline PS 1,2 . & ... presenilin proteins 1,2 \\
\hline Pen $2 \ldots \ldots \ldots \ldots$ & ...presenilin enhancer 2 \\
\hline RAGE ......... & ....receptors advanced glycation end products \\
\hline $27-\mathrm{OH} . . . \ldots . .$. & .. 27-hydroxycholesterol \\
\hline & ....unconditioned stimulus \\
\hline
\end{tabular}


Chapter One

Introduction 


\section{LITERATURE REVIEW}

\section{Introduction}

AD was first characterized in the 1900's by Alois Alzheimer. Today AD is a major health problem affecting about $5 \%$ of people age 65 years old and more than $35 \%$ of people over the age of 80 years old (Jaya Prasanthi et al., 2008). Incidence of the disease is thought to reach 81.1 million people by 2040 (Martins et al., 2009). The disease causes severe dementia and is characterized by two neuropathological features (1) neurofibrillary tangles and (2) A $\beta$ plaques. In the past several decades certain factors such as cholesterol, copper, and sulfatides have been linked to $A \beta$ that have all been shown to be interrelated and furthermore have been shown to affect learning and memory.

Cholesterol is a large, hydrophobic, steroid molecule that is important for cell structure, repair and signaling, myelin synthesis, synaptic plasticity, hormone and neurosteriod production as well as bile acid synthesis. However cholesterol is also a major risk factor for atherosclerosis, coronary heart disease, and AD. In fact, a highcholesterol diet has been shown to result in $\mathrm{A} \beta$ deposits - one of the hallmarks of AD. There is a considerable body of data suggesting that dietary cholesterol could be detrimental to learning and memory and there are also data suggesting that it could be beneficial.

Copper is an essential component of many metalloproteins involved in antioxidant defense. It is also important for growth, cardiovascular, endocrine and pulmonary 
function but copper imbalance has been linked to mental retardation, anemia, toxicosis and $\mathrm{AD}$. Copper is involved in $\mathrm{A} \beta$ metabolism and aggregation and appears to be elevated in the blood of Alzheimer's patients, and is one of the trace metals found in $A \beta$ plaques (Reviewed in Hung et al., 2010).

Sulfatides are a class of sulfated galactocerebrosides that have an important role in myelin sheath and axon structure maintenance, modulation of potassium and sodium ion channels, and have been shown to be altered in a number of neurological disorders including; Metachromatic Leukodystrophy (MLD), Parkinson's disease (PD), and AD. The role of sulfatides in $\mathrm{AD}$ is still not fully understood. Most recently it has been suggested that sulfatides are involved in $A \beta$ clearance (Zeng \& Han, 2008). However, the role of sulfatides in learning and memory has not been studied.

\section{Cholesterol synthesis and its regulation}

Cholesterol is a large, hydrophobic, steroid molecule that is essential to the viability of mammalian cells. It is a major component of all cellular membranes; it is involved in synaptic plasticity (Dufour et al., 2006; Koudinov \& Koudinova, 2001) and is a precursor molecule for neurosteroid synthesis (Muaki et al., 2006) and myelin synthesis (Bartzokis et al., 2001) in the brain. There are two sources of cholesterol in the body: diet and natural biosynthesis. It is synthesized via a lengthy process having more than 20 reactions with 3-hydroxy-3-methylglutaryl-CoA reductase (HMG-CoAR) being the rate limiting enzyme (Figure 1.1). Total cholesterol in the body is separated into a central and peripheral pool by the blood brain barrier (BBB). Cholesterol levels in the central 
nervous system (CNS) are tightly controlled and disturbances in cholesterol are correlated with disorders in humans such as Niemann-Pick disease Type C, Smith-LemliOpitz syndrome and AD (Ikonen, 2006; Mori et al., 2001; Papassotiropoulos et al., 2002; Voikar et al., 2002; Whitney et al., 2002; Xie et al., 2000).

All, or nearly all of the cholesterol in the CNS comes from de novo synthesis (Bjorkhem et al., 1998; Danik et al., 1999; Dietschy \& Turley, 2001; Lund et al., 1999; Lutjohann et al., 1996; Spady \& Dietschy, 1983). It is generally thought that mature neurons reduce their endogenous production of cholesterol and instead depend on the production and transfer of cholesterol by glial cells during adult life. Cholesterol is first packaged with ApoE (Apolipoprotein E) and phospholipids, transferred out of glial cells by ATP-binding cassette A1 (ABCA1), and then taken up by neurons through binding to LDL receptor-related protein (LRP) (Shobab et al., 2005). However, recently it was demonstrated that enzymes essential to cholesterol synthesis such as HMG-CoAR are present in adult neurons and are most prominently localized in cortical and hippocampal neurons (Korade et al., 2007). Cholesterol is essential for synaptic plasticity (Frank et al., 2008; Koudinov \& Koudinova, 2001; Koudinov \& Koudinova, 2003; Koudinov \& Koudinova, 2005) and Dufour and colleagues (2006) have illustrated a correlation between an increase in hippocampal brain cholesterol levels and memory changes using rats fed a regular diet. Therefore it is possible hippocampal neurons, as well as cortical neurons are able to synthesize cholesterol in response to new learning, although it is still more likely that glial cells play the major role.

The regulation of CNS cholesterol homeostasis is crucial for proper brain function and is controlled in large part by the enzymatic conversion of cholesterol to 24S- 
hydroxycholesterol which, unlike cholesterol, can leave the brain via the BBB (Bjorkhem et al., 1998; Bretillon et al., 2000; Leoni et al., 2003; Lund et al., 1999; Lutjohann et al., 1996; Meaney et al., 2001; Whitney et al., 2002). There is increasing evidence that neuronal loss and the subsequent breakdown of cholesterol-rich cellular membranes can be detected as an increase in 24S-hydroxycholesterol (Lutjohann et al., 2000; Papassotiropoulos et al., 2002). For example, cholesterol and 24S-hydroxycholesterol levels are elevated in $\mathrm{AD}$ and are correlated with the severity of the disease (Kolsch et al., 2003; Papassotiropoulos et al., 2002; Puglielli et al., 2003; Schonknecht et al., 2002). Furthermore, inhibitors of cholesterol synthesis such as statins can lower cholesterol and 24S-hydroxycholesterol levels in the human CNS (Bocan et al., 1998; Dietschy \& Turley, 2001; Locatelli et al., 2002; Simons et al., 2002b; Vega et al., 2003) and appear to reduce the incidence of $A \beta$ deposition and may decrease the severity of cognitive impairment (Bu, 2009; Buxbaum et al., 2002; Fassbender et al., 2001; Guardia-Laguarta et al., 2009; Rockwood et al., 2002; Simons et al., 2002b; Sjogren et al., 2003; Vega et al., 2003; Wolozin et al., 2000). However, the rate of 24S-hydroxycholesterol output is lower than the level of cholesterol synthesis (Bjorkhem et al., 1998; Dietschy \& Turley, 2001; Xie et al., 2003) suggesting that there may be other pathways for the efflux of cholesterol from the CNS including an ApoE-dependent pathway (Bjorkhem et al., 1998; Bu, 2009; Mahley \& Rall, 2000; Teunissen et al., 2002; Zlokovic, 2005). It is noteworthy that a high-fat, high-cholesterol diet produces alterations in the BBB in ApoE-knockout mice providing evidence that cholesterol and ApoE may play a role in the integrity of the BBB and cholesterol efflux (Mulder et al., 2001). 


\section{Neurogenesis and learning}

It is thought that increases in cholesterol levels are required for adult neurogenesis. There is evidence in the literature both in support and against the role of adult neurogenesis in learning and memory. It has been established that the hippocampus is involved in the learning and memory process and a site of adult neurogenesis (Bangasser et al., 2006; Frankland \& Bontempi, 2005; Runyan et al., 2004). However, the debate now is whether or not newly formed cells develop in the adult hippocampus as a result of learning and whether or not these cells are correlated with learning and memory. A number of studies using trace eye blink conditioning, Morris water maze, and conditioned food preference support the idea that learning increases the number of new neurons (Ambrogini et al., 2000; Dobrossy et al., 2003; Gould et al., 1999; Hairston et al., 2005; Lemaire et al., 2000; Leuner et al., 2004; Olariu et al., 2005; Waddell \& Shors, 2008). Interestingly, studies that use tasks which are not mainly hippocampal dependent do not stimulate neurogenesis (Gould et al., 1999; Van Der et al., 2005). On the other hand there is also evidence against learning induced hippocampal neurogenesis (Snyder et al., 2005; van et al., 1999). Furthermore some studies have even demonstrated a decrease in hippocampal neurogenesis as a function of learning (Ambrogini et al., 2004; Dobrossy et al., 2003; Olariu et al., 2005; Pham et al., 2005).

Given that the hippocampus is a site of neurogenesis, it would be interesting to study levels of lipids such as cholesterol and sulfatides in the hippocampus and other learning related structures as a function of learning. The dentate gyrus of the hippocampus is thought to produce thousands of granule neurons each day (Waddell \& 
Shors, 2008). Many of these cells only survive a short period; however it has been shown that survival is enhanced by hippocampal-dependent trace classical conditioning. Waddell and Shors (2008) conducted an experiment illustrating trace conditioning contributed to increased survival of cells in the dentate gyrus. Furthermore, their work also suggested the duration of hippocampal activation plays a factor in enhancing survival of newly generated cells in the dentate gyrus. Hypothetically, increases in survival of neurons would lead to increases in hippocampal brain cholesterol.

Some studies have suggested that the number of new neurons is closely correlated with learning. The positive correlation was demonstrated by experiments involving the song systems of canaries (Goldman \& Nottebohm, 1983; varez-Buylla et al., 1990) and seed storage and retrieval behavior in chickadees (Barnea \& Nottebohm, 1994). Other studies such as one by Drapeau and colleges showed a positive correlation between newly formed cells and learning (Drapeau et al., 2003) as did another by Waddell and Shors (2008). Furthermore studies that used drugs known to decrease neurogenesis all resulted in impaired learning of various tasks (Matthews et al., 2008; Matthews \& Silvers, 2004; Spain \& Newsom, 1991). However, factors such as estrogen which are thought to increase neurogenesis (Tanapat et al., 1999) have demonstrated both positive effects on learning (Luine et al., 1998; Luine, 2007) as well as negative effects (Holmes et al., 2002). Hence, it is important to study other factors that affect learning to better understand the mechanisms of neurogenesis. 


\section{Dietary cholesterol and learning}

There have been conflicting findings on the effects of dietary cholesterol on learning and memory. On the one hand, there are many human and animal studies that suggest high cholesterol levels negatively affect learning and memory. Humans studies report that high serum cholesterol is a risk factor for mild cognitive impairment and dementia (Foster, 2006; Kivipelto et al., 2001; Näslund et al., 2000; Solomon et al., 2007; Solomon et al., 2009; Teunissen et al., 2003; Yaffe et al., 2002). Furthermore, it has been shown that cholesterol levels are correlated with measures of intelligence (Atzmon et al., 2002; Muldoon et al., 1997; Reitan \& Shipley, 1963; van Exel et al., 2002; Yaffe et al., 2002). In addition, many studies have suggested a strong relationship between cholesterol levels and AD (Canevari \& Clark, 2007; Evans et al., 2000; Hartmann, 2001; Jarvik et al., 1995; Ledesma \& Dotti, 2006; Lesser et al., 2009; Notkola et al., 1998; Simons et al., 2001; Sjogren et al., 2006; Stewart et al., 2001). Cholesterol lowering drugs such as statins have been suggested to help reduce the rate of cognitive decline in AD patients (Hoglund et al., 2005; Hoyer \& Riederer, 2007; Sparks et al., 2006a; Zandi et al., 2005). Animal studies that supplement dietary cholesterol or give drugs to alter cholesterol metabolism have provided further evidence for a relationship between cholesterol and memory. For example, decreasing cholesterol in aged animals improves learning and memory for tasks such as the Morris water maze (Kessler et al., 1986; Yehuda et al., 1998; Yehuda \& Carasso, 1993). Feeding mice a 2\% cholesterol diet for eight weeks resulted in deficits in working memory in the Morris water maze (Thirumangalakudi et al., 2008). Rats, mice and rabbits given calcium channel blockers 
that reduce the esterification of cholesterol and increase hydrolysis of existing cholesterol esters (Nayler, 1999; Schachter, 1997) demonstrate improvements in learning and memory (Deyo et al., 1989; Kane \& Robinson, 1999; Quartermain, 2000; Woodruff-Pak et al., 1997). Animals given statins also show improved learning and memory (Li et al., 2006).

On the other hand, there are also human and animal studies showing increasing cholesterol levels improve learning and memory. Human studies have shown that high cognitive functioning is correlated with high cholesterol (Elias et al., 2005; Panza et al., 2006). It has also been suggested that cholesterol may protect against cognitive decline (Mielke et al., 2005; Panza et al., 2006; van den Kommer et al., 2009; West et al., 2008). Cholesterol manipulation in animals has also provided support for the hypothesis that cholesterol improves learning and memory (Miller \& Wehner, 1994; Upchurch \& Wehner, 1988). Dufour and colleagues have shown feeding rats $2 \%$ cholesterol enhances water maze learning (Dufour et al., 2006). Animals that are either deficient in cholesterol or have cholesterol synthesis blocked have problems with learning and memory (Endo et al., 1996; O'Brien et al., 2002; Voikar et al., 2002; Xu et al., 1998) but these learning deficits are reversed by feeding rats cholesterol (Xu et al., 1998). Cholesterol can significantly increase acquisition of trace conditioning of the rabbit nictitating membrane response (NMR) (Schreurs et al., 2003; Schreurs et al., 2007a; Schreurs et al., 2007b) and heart rate (Schreurs et al., 2007c). Many of these positive effects of cholesterol may be due to cholesterol being crucial to the formation of new synapses, normal receptor function and synaptic plasticity (Dufour et al., 2006; Goritz et al., 2002; Koudinov \& Koudinova, 2001; Mauch et al., 2001; Mitter et al., 2003; Pfrieger, 2003a; Pfrieger, 
2003b; Sooksawate \& Simmonds, 2001a; Sooksawate \& Simmonds, 2001b; Wasser et al., 2007).

There are several hypotheses to explain dietary cholesterol's effect on learning and memory summarized in Figure 1.2. First, several researchers have demonstrated that although the BBB is usually impermeable to cholesterol, it's integrity is compromised in the cholesterol-fed rabbit (Ghribi et al., 2006a; Sparks et al., 2000a). Second, it has recently been demonstrated that there is a net flux of a cholesterol metabolite, 27hydroxycholesterol (27-OH), from the circulation into the brain in healthy human volunteers. Further, it was demonstrated that in mice a diet rich in cholesterol increased this natural flux of 27-OH into the brain (Heverin et al., 2005). 27-OH has been shown to be an activator of transcription factors, such as liver X receptors (LXR) that regulate the expression of ApoE and ABCA1 both of which play important roles in cholesterol transport (Abildayeva et al., 2006). In addition, 27-OH has been shown to bind to and modulate estrogen receptors. Estrogen receptors have been shown to stimulate mitogenactivated protein kinase (MAPK) as well as phosphatase pathways that could modulate $N$-methyl $D$-aspartate (NMDA) receptors and $\alpha$-amino-3-hydroxy-5-methyl-4isoxazolepropionic acid (AMPA) receptors (Hojo et al., 2008). Third, it is possible that low density lipoprotein receptors (LDLR) provide an avenue for dietary cholesterol to affect learning and memory (Herz \& Chen, 2006; Qiu et al., 2005). These receptors are known to regulate cholesterol transport across the BBB and to be involved in learning and memory (Cao et al., 2006). Recently it was demonstrated that cholesterol-fed rabbits alter expression of other receptors involved in cholesterol and $A \beta$ transport. Specifically, Prasanthi and colleagues (2008) showed a decrease in expression of LRP-1 and an 
increase in the expression of receptors for advanced glycation end products (RAGE) as well as a decrease in levels of insulin degrading enzyme (IDE), also known to be an A $\beta$ degrading enzyme. These changes would decrease the transport of $A \beta$ and cholesterol out of the brain while increasing $A \beta$ levels in the brain. Fourth, it is possible that certain proinflammatory cytokines, such as members of the interleukin family are induced by a high cholesterol diet and are then able to affect learning and memory (Kaul, 2001; Rahman et al., 2005). Injections of interleukin $1 \beta$ have been shown to facilitate trace conditioning in rats (Servatius \& Beck, 2003). Fifth, there is evidence that the number of A $\beta$-labeled neurons increases in the brain of cholesterol-fed rabbits (Schreurs et al., 2003; Schreurs et al., 2007b; Sparks, 1996). Research has shown that $A \beta$ is normally present in the brain and one of its functions is to modulate potassium channels (Pearson \& Peers, 2006). These potassium channels have been shown to mediate synaptic plasticity and learning (Plant et al., 2006). Therefore, modulation of these channels may lead to enhanced learning in the cholesterol-fed rabbit. The processing of $A \beta$ from amyloid precursor protein (APP), an integral membrane protein, by beta-secretase, also an integral membrane protein, is influenced by cellular membrane composition. The processing of $\mathrm{A} \beta$ has been hypothesized to occur mostly in lipid rafts, cholesterol enriched microdomains within cellular membranes. The composition of lipid rafts allows for increased interaction of integral-associated processing. It has been theorized that a high cholesterol diet allows for some cholesterol crossing the BBB resulting in the increased uptake and transport of cholesterol by astrocytes to neurons (Figure 1.3). This increase of cholesterol in neuronal cellular membranes would increase the presence of lipid rafts and processing of integral-associated processing such as A $\beta$ (Liu et al., 2009; Shobab et al., 2005). Sixth, 
it has been demonstrated that dietary manipulation can alter neuronal membrane composition (Faulks et al., 2006). Ghribi and colleagues (2006b) demonstrated that although feeding rabbits a $2 \%$ cholesterol diet does not increase overall brain cholesterol levels, it does increase hippocampal neuronal membrane cholesterol levels. More recently it has even been demonstrated that lipid alterations are evident in AD cortex (Martin et al., 2009). Therefore, it is possible that alterations of cholesterol homeostasis in the CNS may affect the composition of neuronal membranes by increasing or decreasing other membrane components to maintain rigidity and function (Hayashi et al., 2002).

\section{Amyloid beta}

Virchow first established the term amyloid in the $19^{\text {th }}$ century to refer to chunks of debris invading different tissues (Gandy, 2005). The main component of amyloid deposits is a 39-43 amino acid protein called $A \beta$ (Masters et al., 1985). $A \beta$ is normally metabolized from APP, which is ubiquitous among cells in the body (Kang et al., 1987). In the brain, a maximal amount of $A \beta$ is generated from neuronal cells and aggregated into senile plaques (another name referring to amyloid deposits) (Glenner \& Wong, 1984b; Masters et al., 1985; Seubert et al., 1993). Although A $\beta$ deposits are present in normal healthy aging individuals (Davies et al., 1988; Roberts et al., 1991), there is a substantial body of evidence linking A $\beta$ to neurological disorders (Glenner \& Wong, 1984a). Since the first description of A $\beta$, a controversy surrounding its role in the CNS has been developing. In light of the work in the field, it appears that $A \beta$ plays a physiological role that can turn pathological in the excess presence of substances that 
bind to and react with it. This paradox has led to the focus on substances, such as certain metals, that play a role in the aggregation and toxicity of $A \beta$.

\section{Amyloid beta synthesis}

The processing of A $\beta$ from APP is well understood. The particular secretases and their cleavage sites have been well characterized. As illustrated in Figure 1.4 APP can undergo initial cleavage by one of two competing secretases, designated alpha and beta-secretase. Alpha-secretase activity is mediated by any one of the three members of the ADAMs (a disintegrin and metalloproteinase) family, namely ADAM-9, ADAM-10 (also known as TNF- $\alpha$ converting enzyme or TACE) and ADAM-17. If APP undergoes initial processing by alpha-secretase then soluble alpha-APP and C83 are generated. Alpha-secretase cleaves APP in the middle of the A $\beta$ domain between Lys +16 and Leu +17 , preventing the formation of $A \beta$. On the other hand, processing by betasecretase, a membrane bound aspartyl protease, results in soluble beta-APP and C99 (Vassar et al., 1999). Beta-secretase, also known as BACE1 (for $\beta$-site APP Cleaving Enzyme 1), cleaves APP to form the N-terminal of $A \beta$ at Asp +1 . The carboxyterminal fragment, C99, is a substrate for gamma-secretase, a complex that includes presenilin proteins (PS1 and PS2), nicastrin (NCT), anterior pharynx-defective (Aph1), and presenilin enhancer 2 (Pen2). C99 cleavage results in A $\beta$ and the APP intracellular domain (AICD). However this cleavage is not precise and sometimes results in $\mathrm{A} \beta$ of varying lengths. Of the two main species of $A \beta$ ( $A \beta 1-40$ and $A \beta$ 1-42), $A \beta$ 1-40 is predominately found in biological fluids associated with lipoprotein particles while $\mathrm{A} \beta$ 1- 
42 is predominately found in senile plaques (Koudinov et al., 1996; Masters et al., 1985).

Further association between monomer $A \beta$ peptides results in oligomers, the form thought to be most responsible for neurotoxic effects (Barghorn et al., 2005; Dahlgren et al., 2002; Lublin \& Gandy, 2010; Manelli et al., 2007; Nimmrich et al., 2008). The end product, spherical amyloid deposits or plaques, is actually thought to be inert. However, the question of whether $A \beta$, aggregated or unaggregated, actually functions in preventing toxicity or whether it is the source of toxicity is heavily debated (Reviewed in Gandy, 2005; Keshet et al., 2010; Kojro \& Fahrenholz, 2005; Vassar, 2005).

\section{Aß roles and interaction with metals}

$\mathrm{A} \beta$ has been the subject of a long-standing debate among the neuroscientific community. Some believe it serves a neuroprotective role while others believe it to serve more of a neurodestructive role (Ramsden et al., 2001; Yankner et al., 1989). Indications that $\mathrm{A} \beta$ may play both a neuroprotective and physiological role are suggested by beneficial roles of APP and its by-products. In vivo unprocessed APP has been shown to have a role in axonal transport of membrane-associated cargo (Kamal et al., 2001). It has also been shown to be involved in enhancing neurite outgrowth (Milward et al., 1992). The by-product of alpha-secretase cleavage (alpha-APP) has been shown to have neuroprotective properties (Caille et al., 2004). Furthermore, the by-product produced in the cleavage of $\mathrm{C} 99$ by gamma-secretase, AICD, has been shown to serve a role as a transcription factor for gene expression (Cao \& Sudhof, 2001). Studies focused on A $\beta$ further suggest a physiological role. Studies have shown a possible role for unaggregated 
$A \beta$ in modulating ion channel activity (Ramsden et al., 2001). Ramsden and colleagues experimented with only a small concentration of unaggregated $A \beta \quad 1-40$ and not the more neurotoxic $A \beta 1-42$. This leaves the possibility that one species may play a physiological role while the other plays a neurotoxic one. In a study by Kamenetz and colleagues, there is a suggestion that $\mathrm{A} \beta$ serves a role in subduing neuronal over excitability. They demonstrated the presence and function of $A \beta$-suppressing excitatory synaptic transmission in healthy neurons (Kamenetz et al., 2003). A malfunction in this physiological role for $A \beta$ could theoretically lead to high levels of neuronal activity and neurotoxicity. $A \beta$ has also been shown to be essential for the viability of neurons in culture (Plant et al., 2003; Plant et al., 2006). In addition, other experimental data raise the possibility that $A \beta$ may serve a protective role in regulating oxidative damage by chelating redox-active metals (Atwood et al., 1998; Yankner et al., 1990; Zou et al., 2002). Low doses of $A \beta$ have even been suggested to play a role in memory retention and long-term potentiation (Morley et al., 2010).

$A \beta$ has also been associated with neurodestruction. The first description of the toxic properties of $A \beta$ was described in a study performed by Yankner and colleagues in 1989. They demonstrated that cells transfected with human APP degenerated more quickly than control cells (Yankner et al., 1989). Since then, many studies have characterized its pathological nature. Experimental evidence in recent years has shown that soluble dimeric and oligomeric forms of $A \beta$ are more toxic than the monomeric form and are probably the culprits in the pathology of neurological diseases (Cleary et al., 2005; Lublin \& Gandy, 2010; Walsh et al., 2002; Zou et al., 2002). A $\beta$ 1-42 has been shown to be more toxic than $A \beta 1-40$ due to its greater redox potential. This results in an 
increased production of hydrogen peroxide and ultimately oxidative damage (Huang et al., 1999; Rottkamp et al., 2001; Taddei et al., 2010). Most of the toxicity induced by A $\beta$ is thought to be a result of its oxidative properties. Research has also demonstrated $A \beta$ peptides form ion channel pores in neurons. This is thought to allow the passage of ions such as calcium and disturb homeostasis levels leading to neuronal death (Arispe et al., 1993; Parodi et al., 2010; Simakova \& Arispe, 2006). A $\beta$ has also been shown to activate the innate immune system and cause inflammation (Salminen et al., 2008). Most recently it has been suggested that $A \beta$ oligomers induce a significant impairment in memory consolidation and loss of synapses in the dentate gyrus (Freir et al., 2010).

Based on the findings reviewed here, $A \beta$ may have a physiological as well as a pathological role. One possibility is that the line between $\mathrm{A} \beta$ serving a physiological versus pathological role is concentration dependent. If $A \beta$ is over-expressed or not cleared from the CNS, then it becomes neurotoxic from increased monomers associating into oligomers. Questions remain about the nature of the mechanism underlying the malfunction that occurs when $A \beta$ switches from having a physiological role to promoting neurological pathology. Evidence suggests CNS elevations of metal ions in neurological disorders are associated with both increased $A \beta$ deposits and production of hydroxyl radicals. Hence, attention has been directed to the relationship between metals and $A \beta$ to uncover a potential link between the two.

It has been known for some time that trace metals, in particular copper and zinc, play a major role in the aggregation of A $\beta$ (Atwood et al., 1998; Atwood et al., 2000; Bush et al., 1994b; Bush et al., 1994a; Huang et al., 2004a; Mantyh et al., 1993). A $\beta$ has binding sites for metal ions that allow for peptide aggregation via a metal ion 
intermediate (Atwood et al., 1998; Bush et al., 1994a; Curtain et al., 2001). There are two important sites in $A \beta$ that are essential for metal binding and reduction. The first site, located in the hydrophilic $\mathrm{N}$-terminal half of the peptide, includes three histidine residues on $\mathrm{A} \beta$ at positions 6,13 , and 14 and one tyrosine residue at position 10 . This site is thought to be involved in metal ion binding (Atwood et al., 1998; Curtain et al., 2001; Liu et al., 1999). These residues have also long been known to be effective chelators of transitional metals (Lovstad, 1987). Using nuclear magnetic resonance and electron paramagnetic resonance spectroscopy, researchers have determined that the binding site of copper in $A \beta$ includes those residues described above (Curtain et al., 2001; Liu et al., 1999; Yang et al., 2000). The importance of these residues has been emphasized in studies where their absence resulted in a diminished capacity for copper-, zinc-, or ironinduced A $\beta$ aggregation (Atwood et al., 1998; Curtain et al., 2001; Liu et al., 1999). Curtain et al (2001) proposed a model of $\mathrm{A} \beta$ aggregation mediated by $\mathrm{Cu}^{2+}$ through histidine bridging. Histidine bridging could be mediated by any imidazole ring of a histidine residue resulting in the aggregation of multiple monomeric $\mathrm{A} \beta$ species.

The second site involves a single residue, methionine (Met) 35, located in the lipophilic C-terminal portion of the peptide. This residue is thought to be crucial for reduction of metals. The importance of Met 35 for the redox ability of $A \beta$ was examined in the presence of copper. Experiments using truncated $\mathrm{AB}$ peptides were shown to have both a reduction in their capacity to bind copper and in their production of hydrogen peroxide (Butterfield et al., 2010; Curtain et al., 2001).

Any condition that promotes an increase in metal ion concentrations provides an ideal setting for $\mathrm{A} \beta$ aggregation. Elevated concentrations of metal ions in the CNS are 
also believed to play a role in the pathology of neurological disorders such as Amyotrophic lateral sclerosis, PD, and AD (Cornett et al., 1998; Frederickson, 1989). The concentrations of trace metals are increased in AD patients, especially in the cortex and hippocampus, and are localized in the areas nearest to plaques (Lovell et al., 1998). Levels of zinc, copper, and iron are more than doubled compared to controls and further elevated in the core and periphery of senile plaques (Lovell et al., 1998). Additional evidence for metals playing a role in $A \beta$ aggregation comes from experimental evidence demonstrating solubilization of $\mathrm{A} \beta$ from postmortem $\mathrm{AD}$ brain tissue by metal chelators (Bush, 2002; Cherny et al., 2001).

Other researchers have demonstrated that copper may in fact promote $A \beta$ synthesis. Copper chelation in $\mathrm{AD}$ transgenic mice inhibited $\mathrm{A} \beta$ accumulation (Cherny et al., 2001). In another study elevated copper and cholesterol levels in rabbits by dietary supplementation resulted in increased A $\beta$ deposition (Sparks \& Schreurs, 2003). More research is needed to resolve conflicting views regarding increased copper levels and APP processing. It is known that the copper-binding site on APP encompasses the domain of amino acids within the span of 135-155 (Simons et al., 2002a). Having characterized the structure of the copper binding site in APP, Barnham et al (2003) showed that a good deal of homology existed between it and the copper binding sites on copper chaperones. It was even demonstrated that there might exist some sort of feedback mechanism between APP gene expression and copper levels. Upon intracellular copper depletion, APP gene expression was down regulated (Bellingham et al., 2004). 


\section{Sulfatides and their role in learning}

In a recent study, $A \beta$ clearance has been suggested to be mediated by the sulfate galactose moiety of a particular sphingolipid (Zeng \& Han, 2008). Sphingolipids are one of three main classes of lipids within cellular membranes. Their glycosylated derivatives are called glycosphingolipids which are classified into two different classes based on the first sugar (either glucose or galactose) attached to the ceramide backbone. Galactosecontaining glycosphingolipids can then be sulfated and become sulfatides (also spelled sulphatides). The biosynthesis and degradation of sulfatides is summarized in Figure 1.5. Sulfoglycolipids were first described by Thudichum in the late $19^{\text {th }}$ century (Thudichum, 1884). Sulfatides, both in the central and peripheral nervous system, are a main component of the myelin sheath. Sulfatides are mainly found in oligodendrocytes and Schwann cells. Low amounts of sulfatides have been detected (Berntson et al., 1998; Pernber et al., 2002) and later shown to be synthesized in neurons and astrocytes (Isaac et al., 2006). CGT (ceramide galactosyltransferase), essential for sulfatide synthesis, has been detected in the brainstem and cerebellum (Schaeren-Wiemers et al., 1995). It is possible that it has not been detected in other neuronal types due to very low expression levels. CST (cerebroside sulfotransferase), the rate limiting step for sulfatide synthesis, has been shown to be expressed in many other neuronal cells in the cortex, and hippocampus (Eckhardt et al., 2007). Sulfatides are transported and modulated by ApoE (Han et al., 2003a). Specifically, sulfatides could be imported from astrocytes into neurons via ApoE-mediated endocytosis by LDLR, in much the same way cholesterol is transported (Han, 2007). 
Sulfatides have been shown to have several roles in the nervous system. They are a structural component of myelin (Norton WT \& Cammer W, 1984) and important for its maintenance (Marcus et al., 2006). They play a role in the differentiation of myelinating cells early in development (Bansal et al., 1988; Bansal \& Pfeiffer, 1989), and even play a role in maintaining axon structure (Marcus et al., 2006). Sulfatides have been shown to be novel activators of calcium-sensitive potassium channels that impact synaptic plasticity and learning (Chi \& Qi, 2006). Most recently it has been suggested that sulfatides are involved in A $\beta$ clearance (Zeng \& Han, 2008).

Disturbances to the normal levels of sulfatides in the CNS have shown neurological consequences. An accumulation in sulfatides caused mostly by a deficiency in arylsulfatase A (ASA), an enzyme responsible for the degradation of sulfatides in lysosomes, causes MLD. The major pathological feature of MLD is demyelination resulting in ataxia, epileptic seizures and other neurological symptoms. Late-onset MLD patients also suffer from cognitive decline and deficits in new learning (Sadovnick et al., 1993; Shapiro et al., 1994; Shapiro et al., 1995). ASA-deficient mice have been extensively used to study MLD and have provided insight into the effect of sulfatide accumulation in the brain. Some of these effects include Purkinje cell degeneration (D'Hooge et al., 1999), learning and memory impairments (D'Hooge et al., 2001), and axonal degeneration and cortical hyperexcitability (Ramakrishnan et al., 2007). Elevated levels of sulfatide were also found in PD subjects (Cheng et al., 2003). Reduction of sulfatide levels also has neurological consequences. Sulfatide deficiencies result in impaired maintenance of sodium and potassium channels (Ishibashi et al., 2002), neuronal degeneration (Honke et al., 2002), and reduced axon diameters (Marcus et al., 
2006). Sulfatide level reductions have been reported in a transgenic mouse model of Huntington's disease (Desplats et al., 2007), as well as in transgenic mouse models and human subjects in AD (Cheng et al., 2008; Han et al., 2002; Han et al., 2003b; Han, 2007; Zeng \& Han, 2008).

\section{Myelin composition}

Myelin is a layer formed around axons by membrane extensions of oligodendrocytes in the CNS and Schwann cells in the peripheral nervous system. Its primary function is to allow for fast conduction of action potentials and maintain axonal integrity (Yin et al., 2006). Myelin has a high proportion of lipid content. It is enriched in both cholesterol and sulfatides. Cholesterol accounts for one third of myelin lipids and sulfatides and its precursor $\mathrm{Gal} \mathrm{C}$ also accounts for almost one third of myelin lipids (Eckhardt, 2008). Therefore, separating myelin from non myelin may help in detecting subtle changes in cholesterol and sulfatide levels.

\section{Classical conditioning and the rabbit NMR}

Classical conditioning of the rabbit NMR and eye blink was first reported in the early 1960s by Gormezano who paired a tone conditioned stimulus (CS) with a corneal air puff (AP) unconditioned stimulus (US) (Deaux \& Gormezano, 1963; Gormezano et al., 1962; Gormezano, 1966; Schneiderman et al., 1962). An AP to the cornea usually elicits an eye blink (extension of the nictitating membrane and closure of the eyelid) 
whereas the tone usually does not elicit any response. Animals given paired tone-puff presentations show the emergence of an NMR to tone and a progressive increase in the frequency of CRs across days of training to levels of 50-95\% depending on the difficulty of the training paradigm. In marked contrast, animals given CS-alone, US-alone, or explicitly unpaired CS-alone and US-alone presentations rarely exceed a frequency of $10 \%$ membrane extension on any single day and average a level not appreciably higher than the base rate of $5 \%$.

The neural substrates of NMR/eyelid classical conditioning in the rabbit include the brainstem, cerebellum and hippocampus (Berger et al., 1991; Berger \& Orr, 1983; Cavallaro et al., 2001). The forebrain also plays a role in trace conditioning and extinction (where CSalone trials are presented after conditioning) (Gruart et al., 2000). The involvement of the brainstem and cerebellum is necessary for classical conditioning: however, involvement of the hippocampus in classical conditioning is dependent upon the complexity of the task (Beylin et al., 2001). For example, although the hippocampus may not be necessary for the acquisition of a CR during delay conditioning (where the CS and the US overlap), it is necessary for $\mathrm{CR}$ acquisition during trace conditioning (where there is a temporal gap between the CS and US) (Bangasser et al., 2006).

The role of the hippocampus in learning a difficult behavioral task and the role of the frontal lobe, specifically the prefrontal lobe, in learning and memory have been well documented (Bangasser et al., 2006; Frankland \& Bontempi, 2005; Runyan et al., 2004). Using rabbits, one study illustrated how large bilateral lesions of the hippocampus made before training seriously impaired learning of a difficult task. When the rabbits were 
trained first, lesions made immediately after training abolished the ability to remember the difficult task; however, lesions made 1 month after training had no effect on memory retention of the previously learned difficult task (Thompson \& Kim, 1996). Therefore, even though the hippocampus and frontal lobe are both essential in learning and memory, the hippocampus is more involved in the formation of new memories while the frontal lobe is more involved in the recall of those memories.

\section{Cholesterol-fed Rabbit}

The 8 week cholesterol-fed rabbit has been used as a model of study for atherosclerosis for over 90 years (Bocan et al., 1998). Although blood cholesterol levels reach as high as $2000 \mathrm{mg} / \mathrm{dL}$ in the 8 week cholesterol-fed rabbit which is 10 times what is normally considered unhealthy human cholesterol levels, there are cases of extreme hypercholesterolemia where patients present total cholesterol levels in excess of 2000mg/dL (Turchin et al., 2004). New Zealand rabbits consistently gain weight throughout the 8 week cholesterol feeding period and rarely if ever succumb to the effects of the diet (Schreurs et al., 2007b). In recent years the cholesterol-fed rabbit has been considered a model to study deficits in learning and memory, specifically AD (Sparks\& Schreurs, 2003). Previous work has demonstrated that the cholesterol-fed rabbit exhibits pathology similar to that seen in $\mathrm{AD}$ such as, intraneuronal $\mathrm{A} \beta$ deposition and $\mathrm{ApoE}$ reactivity (Sparks et al., 1995). In addition, the time course of CNS changes in the cholesterol-fed rabbit is quite rapid and can take place in a matter of weeks allowing for a short time course of study (Sparks, 1996). Given that high cholesterol may play a 
significant role in learning and memory and contribute to $\mathrm{A} \beta$ deposition and $\mathrm{AD}$, a cholesterol-fed animal model would be useful for studying the role of elevated/altered cholesterol and lipid compositions in learning and memory. 
Figure 1.1

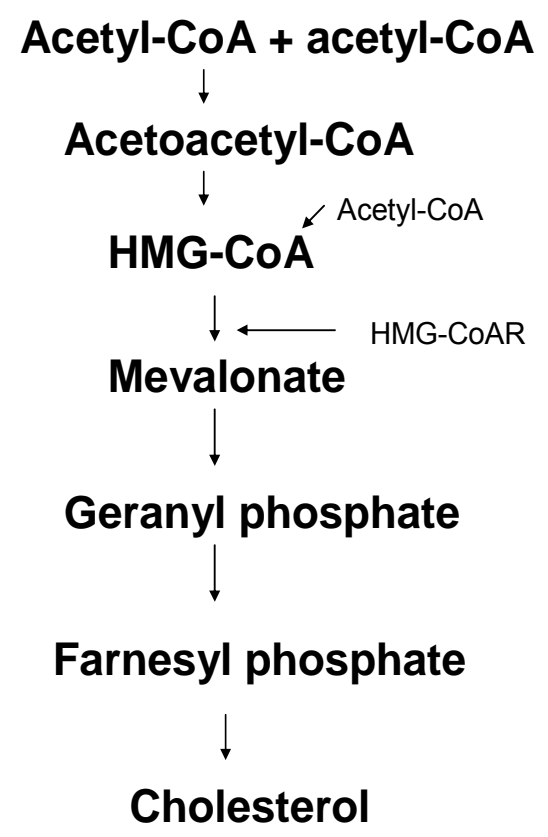

Figure 1.1. Cholesterol Biosynthesis. An abbreviated cholesterol biosynthetic pathway illustrating key steps and enzymes. 


\section{Figure 1.2}

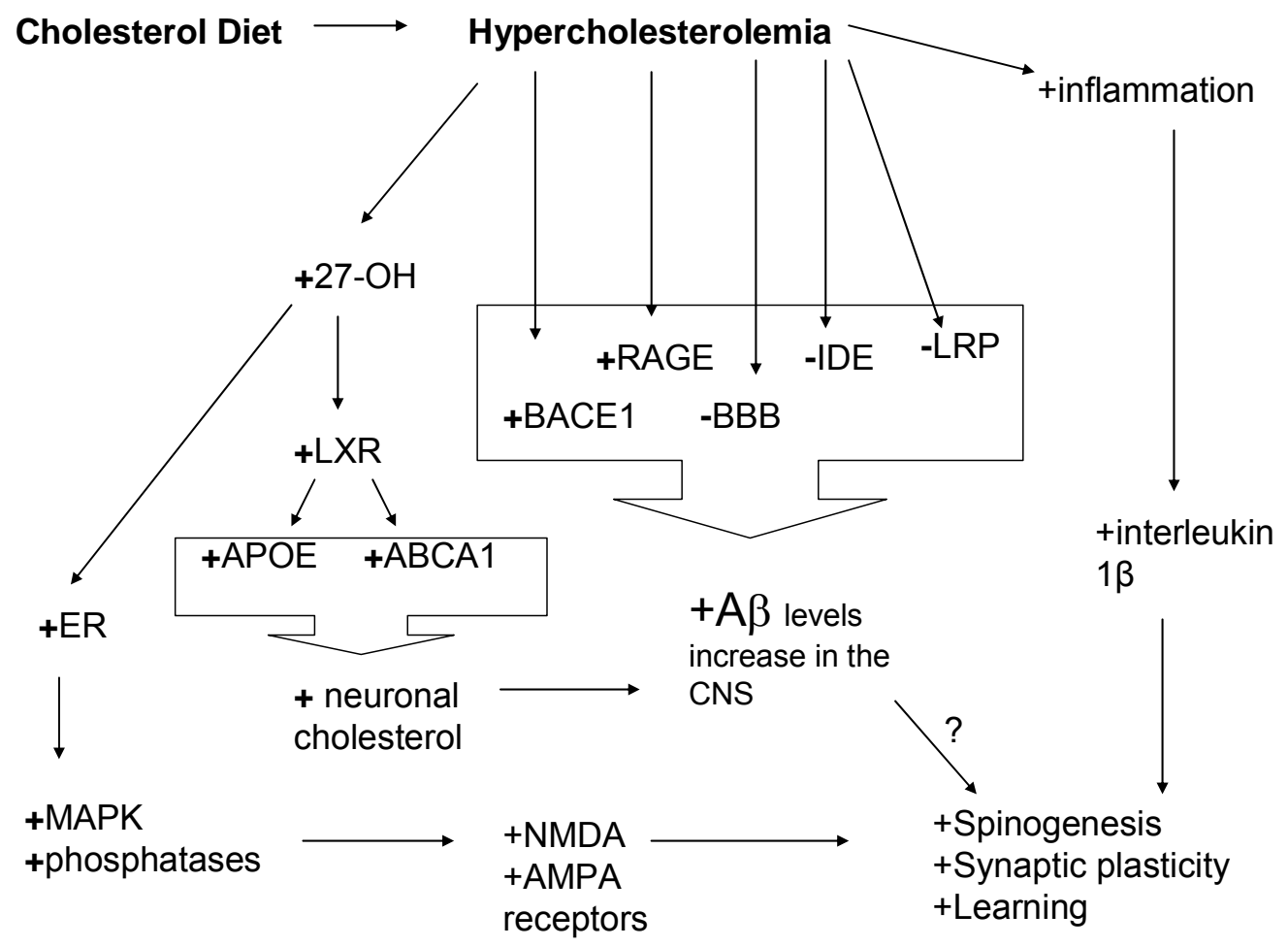

Figure 1.2. Dietary cholesterol has many effects that modulate $A \beta$ levels and learning.

$(+)=$ increases, $(-)=$ decreases or disrupts, $(?)=$ both + and - 


\section{Figure 1.3}

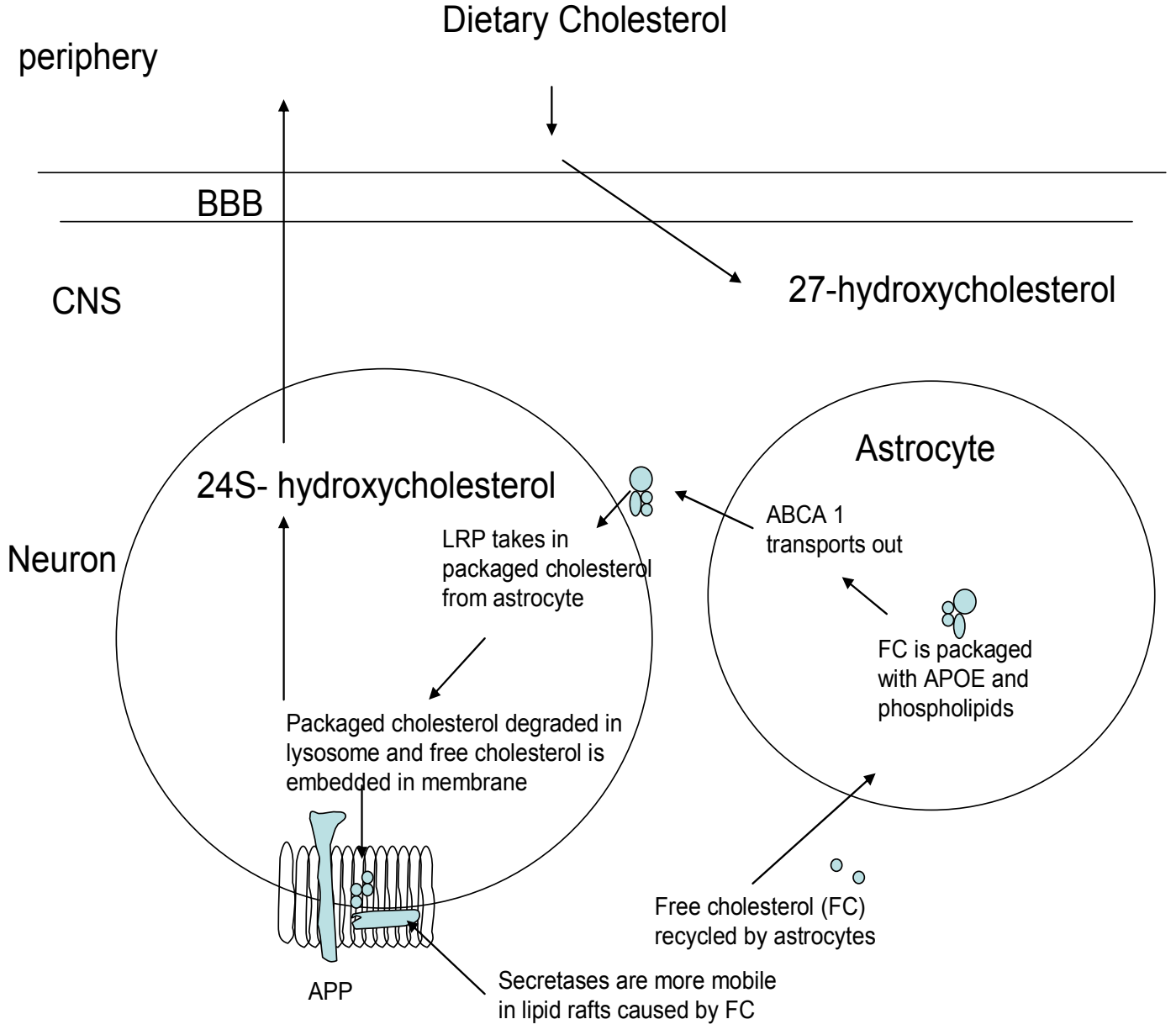

Figure 1.3. The transport of cholesterol between neurons and astrocytes. This transport leads to increased $A \beta$ processing. Hydroxycholesterols mediate cholesterol homeostasis between the brain and the peripheral circulation. 


\section{Figure 1.4}

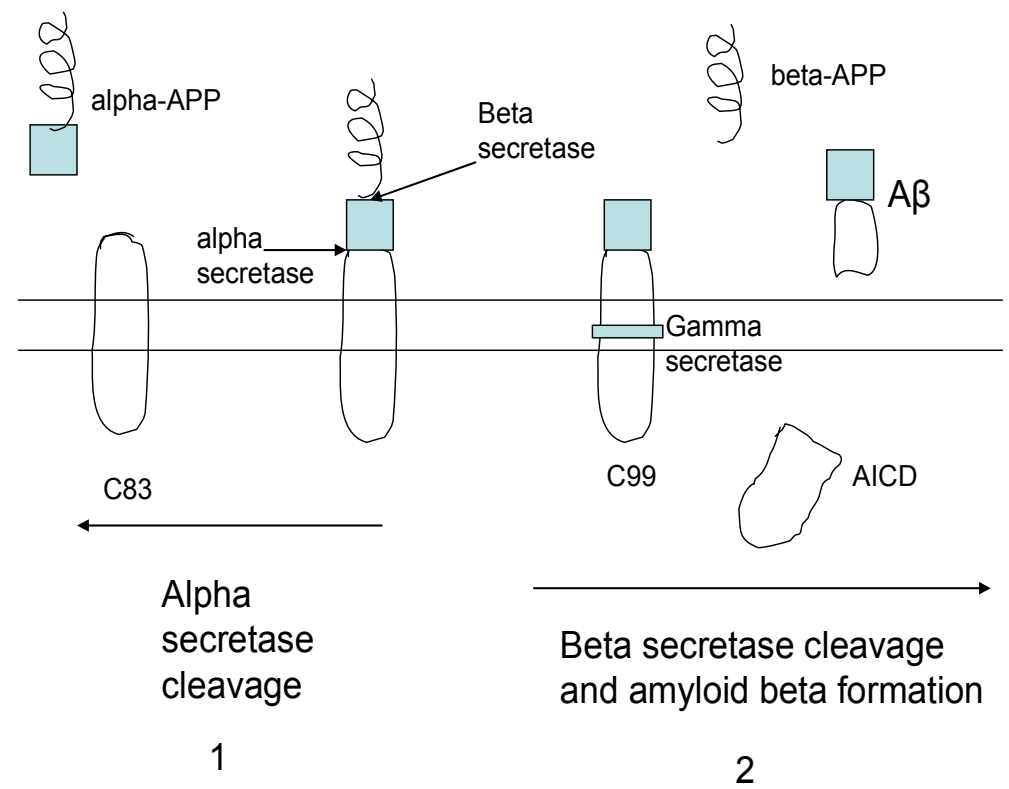

Figure 1.4. APP processing pathways in the brain. One of two competing pathways can occur: Cleavage by alpha-secretase prevents the synthesis of $A \beta$ while cleavage by betasecretase results in the synthesis of $\mathrm{A} \beta$. 


\section{Figure 1.5}

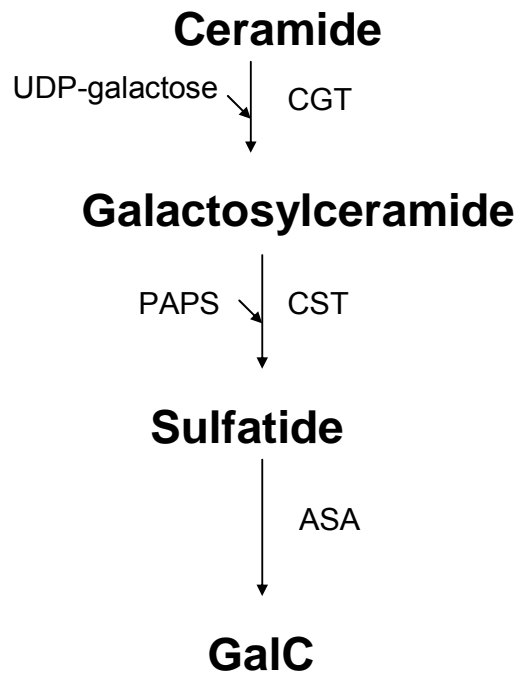

Figure 1.5. The biosynthesis and degradation of sulfatide. Galactosylceramide (GalC) is synthesized in the endoplasmic reticulum by the transfer of galactose to ceramide, which is catalyzed by ceramide galactosyltransferase (CGT). In the Golgi apparatus, GalC is converted to sulfatide by the transfer of a sulfate group from PAPS (3'phosphoadenosine-5'-phosphosulfate) to the galactose residue, which is catalyzed by CST (galactose-3-O-sulfotransferase-1). Degradation of sulfatide in lysosomes starts with the hydrolysis of the sulfate residue by ASA (arylsulfatase A). 
Chapter 2

Objectives and Rationale 


\section{OBJECTIVES}

Study I: Determine if dietary cholesterol and copper effects on learning are a function of cholesterol concentration.

Work conducted previously suggests that dietary cholesterol can either have a positive or negative effect on learning depending on whether or not $\mathrm{A} \beta$ plaques developed. In the earliest study using the cholesterol-fed rabbit it was suggested that dietary cholesterol has a positive effect on learning (Schreurs et al., 2003). A subsequent study however revealed that the combined effect of a cholesterol diet with copper supplementation in the drinking water resulted in a negative effect on learning, as A $\beta$ plaques developed. Therefore, we conducted an experiment with varying concentrations of dietary cholesterol and copper supplemented water in which we assessed learning and the number of immunoreactive $A \beta$ neurons in the hippocampus and cortex. We hypothesized, that $\mathrm{A} \beta$ would increase as a function of cholesterol concentration and learning would improve as a function of cholesterol concentration in the absence of $A \beta$ plaque formation.

\section{Study II: (1) Determine if dietary cholesterol affects memory retention by measuring the rabbit NMR and (2) determine if dietary cholesterol affects brain cholesterol and sulfatide levels.}

Cholesterol is important for cell structure, repair and signaling, hormone production and bile acid synthesis; however, it is a major risk factor for atherosclerosis, 
coronary heart disease, and stroke and has also in recent years been considered a major risk factor for AD. Sulfatides are a class of sulfated galactocerebrosides that have an important role in myelin sheath and axon structure maintenance, modulation of potassium and sodium ion channels, and have been shown to be altered in a number of neurological disorders including AD. The effect of dietary cholesterol on learning and memory remains unclear. There is research demonstrating both positive and negative effects of dietary cholesterol on learning and memory. Furthermore, the relationship between dietary cholesterol and brain cholesterol still remains unclear and the relationship between dietary cholesterol and brain sulfatides has not been studied. In all our previous studies we have assessed dietary cholesterol effects on the formation of a new memory and have not explored the dietary cholesterol's effects on a previously learned taskreferred to as memory recall or retention. Therefore, in this study we investigated the effect of a cholesterol diet on memory recall of a previously learned task and also measured brain cholesterol and sulfatide levels. We hypothesized that dietary cholesterol will have an adverse affect on memory retention and levels of brain cholesterol and sulfatide levels would be influenced by learning or diet.

Study III: Determine if learning- and memory-specific increases in brain cholesterol and sulfatide levels would manifest from a learning task alone or required a combination of both learning and memory tasks.

The results from Study II suggested an increase in brain sulfatide and cholesterol levels as a direct function of associative learning and memory recall. In Study II, the animals were exposed to several training paradigms (both trace conditioning and 
extinction) and all perfused after assessing memory recall. Given that these training paradigms are both hippocampal and prefrontal cortex-dependent (Green \& Arenos, 2007; Moyer, Jr. et al., 1990; Port et al., 1986; Weiss et al., 1999), we investigated whether the learning-associated variations in brain cholesterol and sulfatide levels were a direct function of trace conditioning. We also investigated whether these changes in brain chemistry occurred a day after training or required a two-month period, as was the case in Study II. We hypothesized that the increases in brain cholesterol and sulfatide levels in Study II were the result of combining trace conditioning and extinction and we should therefore see smaller differences from trace conditioning alone.

\section{RATIONALE}

High cholesterol in the general population is an increasing health problem and it is important to understand how it impacts learning, and if it impacts memory. We know the adverse effects it has on our cardiovascular system from many years of research in humans. Researchers started using the cholesterol-fed rabbit as a model for atherosclerosis. In the past decade the cholesterol-fed rabbit model has also been used as a potential tool for investigating AD. However most of the work using cholesterol-fed rabbits has yielded contradictory findings concerning the impact of dietary cholesterol on learning. Some work has reported a positive effect of dietary cholesterol on learning (the formation of a new memory) while other work has suggested a negative effect. Thus far, we have not examined the cholesterol-fed rabbit model to test memory retention (previously formed memory). It is important to test memory retention to gain an 
understanding of how dietary cholesterol has an impact on individual learning and memory structures.

The rationale for the proposed research is: (1) Cholesterol has an important and essential role in cell signaling and synaptic plasticity and, in recent years, has been shown to impact learning; (2) Sulfatides have an important role in myelin sheath and axon structure maintenance, modulation of potassium and sodium ion channels, and have been shown to be altered in a number of neurological disorders including AD; (3) It is still unclear whether dietary cholesterol supplementation causes increases in brain cholesterol and not known if it has any effect on sulfatide levels; (4) Copper has an essential role in normal nutrition as well as mental retardation and has been shown to increase levels of A $\beta$; (5) Simple dietary manipulations have been shown to elevate cholesterol and induce $A \beta$ accumulation; (6) Sulfatides, $A \beta$ and cholesterol share a link in that they are all transported via ApoE-containing lipoproteins; (7) We have a good understanding of the behavior and substrates of learning and memory in the rabbit; (8) The human and rabbit amyloid precursor protein are identical; and (9) The rabbit would make an ideal animal model for studying the effects of cholesterol and $\mathrm{A} \beta$ on learning and memory, and thus, a potential animal model of AD. Using this model, we may be able to develop new and innovative approaches to the treatment of learning and memory deficits. This work is important because of the impact it will have on understanding the role of dietary cholesterol and $\mathrm{A} \beta$ in learning and memory. Furthermore, it expands our understanding of dietary cholesterol effects on brain cholesterol and sulfatide levels. Last, it explores the relationship learning and memory combined and learning alone have on brain cholesterol and sulfatide levels. 
First, we sought to demonstrate that dietary cholesterol with copper supplemented water increases $A \beta$ levels in learning and memory related structures, resulting in a positive effect on learning in the absence of $A \beta$ plaques. Using different dietary cholesterol concentrations $0,0.5,1.0$, and 2.0 supplemented with 0.12 ppm copper water, we determined the learning effect using rabbit classical conditioning, tested hearing in the cholesterol-fed model, and assessed the number of A $\beta$ immunoreactive neurons in the cortex and hippocampus. The rationale is dietary cholesterol and copper increase $A \beta$ in a cholesterol concentration-dependent manner and if senile plaques are formed this will retard learning; however in the absence of plaques learning is enhanced.

Second, we sought to investigate how dietary cholesterol would affect memory retention in our cholesterol-fed rabbit model, and to investigate if dietary cholesterol would have a direct effect on brain cholesterol and sulfatide levels. The rationale is that dietary cholesterol has an effect on the learning of a new memory so it may also affect previously formed memories. We first investigated the effect of dietary cholesterol on memory retention. Then we measured brain cholesterol and sulfatide levels in the hippocampus, frontal lobe, cerebellum, and brainstem by separating each sample into a myelin and nonmyelin fraction to best uncover any subtle changes. We also explored changes in brain cholesterol and sulfatide levels as a function of learning and memory. The rationale is that both cholesterol and sulfatide levels are abundant in the CNS and have been shown to play a role in learning and memory.

Last, we sought to investigate the relationship, if any, between trace conditioning (a learning task) and brain cholesterol and sulfatide levels. The rationale is in Study II we demonstrated brain cholesterol and sulfatide levels change from the combined effect of 
trace conditioning and extinction (memory retention task). We wanted to determine if changes in brain cholesterol and sulfatide levels would present from trace conditioning alone. Also we wanted to explore if these effects were time sensitive. Previously, in Study II, all the tissue was collected several months after the trace conditioning period of the experiment. Here we explore changes in brain cholesterol and sulfatide levels as a function of trace conditioning and time. 
Chapter Three

Preliminary Data: Effects of dietary cholesterol on learning and memory retention 


\section{Introduction}

Our preliminary experiments were designed to determine whether a $2 \%$ cholesterol diet: (1) affected classical conditioning of the rabbit NMR, (2) affected extinction of the rabbit NMR, and (3) had an impact on central cholesterol levels. We will first present data from a classical conditioning experiment in which rabbits were fed a standard $0 \%$ or $2 \%$ cholesterol diet with distilled water for 8 weeks after which levels of acquisition were assessed. Next, we will present data from another classical conditioning experiment in which rabbits were first trained, then fed a standard $0 \%$ or $2 \%$ cholesterol diet and distilled water for 8 weeks and then memory retention of the initially learned task was assessed through extinction. After extinction, these animals received paired training to assess if the cholesterol diet still facilitated learning. At the end of classical conditioning, several of these animals were used to quantify cholesterol levels in several brain regions. From these studies we will provide evidence that cholesterol enhances classical conditioning of the rabbit NMR; however, it appears to have a detrimental effect on memory retention and there is a suggestion of myelin degeneration as a result of the $2 \%$ cholesterol diet. 


\section{Summary}

\section{Experiment 1}

In the first experiment rabbits were classically conditioned using a trace conditioning procedure which involved a $100-\mathrm{ms}$ tone with a $500 \mathrm{~ms}$ trace followed by a 100-ms AP for eight days and then a delay AP conditioning procedure (400-ms tone that co-terminates with a 100-ms AP) for four days. The experiment included two groups; one group received a $2 \%$ cholesterol diet $(n=5)$ and the other a normal $0 \%$ cholesterol Purina $\operatorname{diet}(n=7)$, both having free access to distilled water. As seen in Figure 3.1, the 2\% cholesterol-fed rabbits learned better than the group fed normal chow. A repeated measures analysis of mean percent conditioned responses (CRs) across the eight days of trace conditioning revealed a main effect of days, $[\mathrm{F}(7,70)=20.241, \mathrm{p}<.001]$, and a main effect of group, $[\mathrm{F}(1,10)=5.910, \mathrm{p}<.05]$, as well as an interaction of day and group, $[\mathrm{F}(7,70)=4.286, \mathrm{p}<.05]$. A repeated measures analysis of mean percent $\mathrm{CRs}$ across the four days of delay conditioning did not reveal any significant interaction within or between groups. From this experiment we gathered that a $2 \%$ cholesterol diet enhanced learning and this effect was significant in a hippocampal-dependent trace task. We also learned that the effects of dietary cholesterol are evident in an easier trace conditioning task than that used in the first Schreurs (2003) study where animals were exposed to a longer inter-stimulus $(800 \mathrm{~ms})$ interval between the CS and US. 


\section{Experiment 2}

In the second experiment, six rabbits were classically conditioned to nearly $100 \%$ mean percent $\mathrm{CR}$ using a delay conditioning procedure which involved a $400-\mathrm{ms}$ tone co-terminating with a $100 \mathrm{~ms}$ shock. The rabbits were then placed on either a normal $0 \%$ $(n=2)$ or $2 \%$ cholesterol diet $(n=4)$ for 8 weeks after which memory retention of the initially learned task was assessed through extinction (100-ms tone alone trials). After 6 days of extinction the animals were classically conditioned using a trace conditioning procedure which involved a $250-\mathrm{ms} 5 \mathrm{~Hz}$ tone with a $250 \mathrm{~ms}$ trace followed by a $100-\mathrm{ms}$ corneal AP for 4 days. As depicted in Figure 3.2, it appears that the normal diet group retained a higher memory of the previously learned task than the $2 \%$ cholesterol group. This was the first evidence we saw of memory impairment in the cholesterol-fed rabbit model. The small sample sizes prevented us from making any firm conclusions.

Although most of the tissue from the pilot animals was poorly perfused due to faulty spinal taps, one animal from the $2 \%$ cholesterol group was perfused well enough for biochemical analysis. Using this animal and another animal that received a normal diet from a different experiment we isolated myelin and quantified cholesterol. The methods used for myelin isolation, protein determination, lipid extraction and cholesterol and sulfatide quantification are described in later chapters.

The results were interesting, there seemed to be notable myelin degeneration in the cholesterol fed animal. Furthermore the decrease in myelin levels seem to be proportional to the myelin richness of the area examined. The three areas we 
examined were the prefrontal lobe, brainstem, and cerebellum. We found that there was approximately $25 \%$ decrease in the brainstem, about $6 \%$ decrease in the cerebellum, and less than $2 \%$ decrease in the prefrontal lobe myelin in the cholesterol-fed rabbit compared to the normal-fed rabbit. These results are based on one animal from each group so no significance could be demonstrated. Nonetheless the findings were interesting and warranted further investigation. 


\section{Discussion}

Experiment 1 replicated our previous findings that dietary cholesterol enhances classical conditioning of the rabbit NMR. Interestingly, Experiment 2 revealed that dietary cholesterol may have a detrimental effect on memory retention and suggested myelin degeneration as a result of the $2 \%$ cholesterol diet. The data gathered here are only preliminary; however, the suggestion that dietary cholesterol may have a positive effect on learning of trace conditioning task and then have the opposite effect on a memory retention task warrants further investigation. Moreover, a clue to the mystery maybe linked to the differences between cholesterol myelin levels in certain learning and memory related structures. 


\section{Figure 3.1}

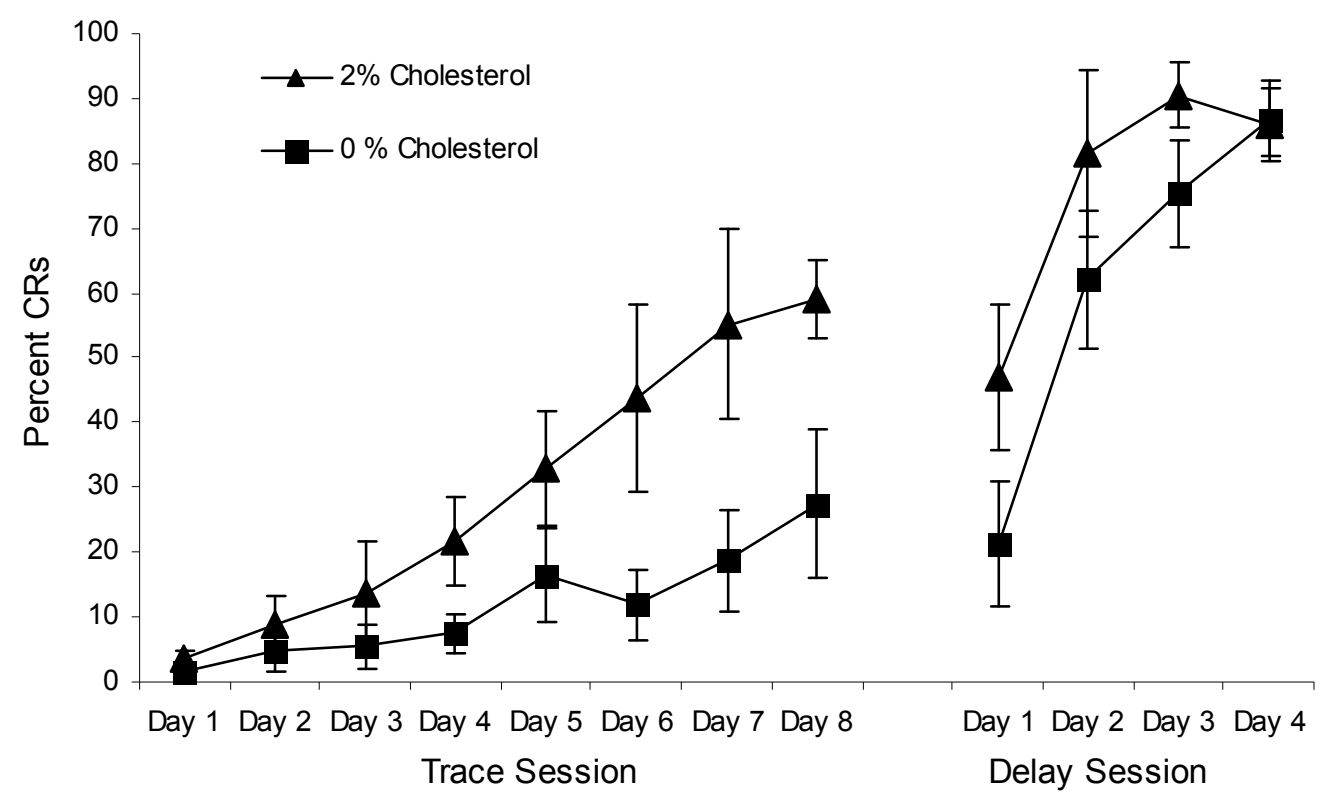

Figure 3.1. Dietary cholesterol enhances learning. Mean percent conditioned responses across days of conditioning. Rabbits were classically conditioned using a trace conditioning procedure which involved a $100-\mathrm{ms}$ tone with a $500 \mathrm{~ms}$ trace followed by a 100-ms corneal AP for eight days and then a delay AP conditioning procedure (400-ms tone that co-terminates with a 100-ms AP) for four days. 


\section{Figure 3.2}

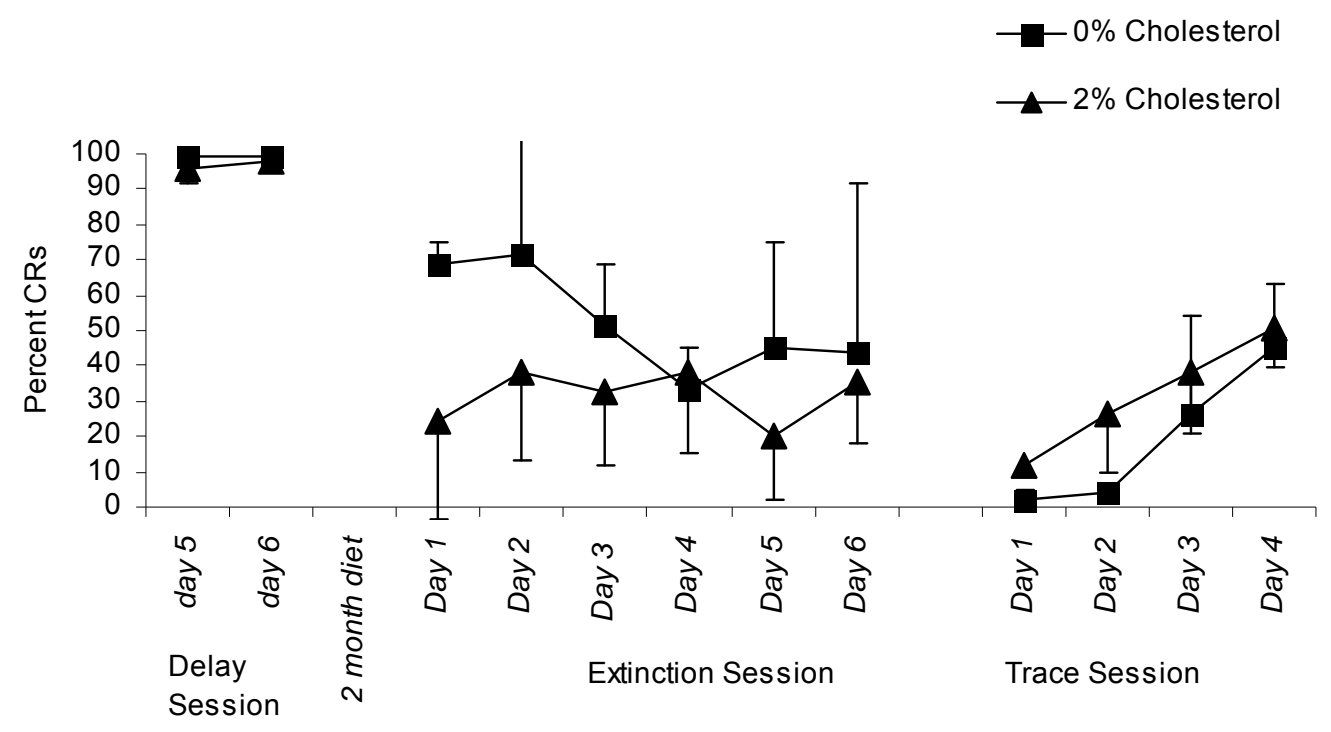

Figure 3.2. Dietary cholesterol impairs memory. Mean percent conditioned responses across days of extinction. Rabbits were previously conditioned to near $100 \%$ CRs. These rabbits were then assigned to either a $2 \%$ cholesterol diet or $0 \%$ normal diet for a period of 2 months and then received extinction (100ms tone alone trials) to assess memory retention. Afterwards rabbits were classically conditioned using a trace conditioning procedure which involved a $250-\mathrm{ms} 5 \mathrm{~Hz}$ tone with a $250 \mathrm{~ms}$ trace followed by a $100-\mathrm{ms}$ corneal AP for four days. 


\section{Chapter Four}

\section{Dietary Cholesterol and Copper Effects on Learning}

This work is part of a publication in the Journal of Nutritional Neuroscience 10: 31-43 (2007). 


\begin{abstract}
Cholesterol supplemented diets have been shown to improve memory tasks in animal models. Rabbits fed varying percentages of cholesterol concentration $(0,0.5,1$, and $2 \%$ ) with $0.12 \mathrm{ppm}$ copper added to the drinking water received airpuff conditioning during which the NMR data was collected. Analysis of A $\beta$ staining, showed a significant cholesterol concentration-dependent increase in the number of $A \beta$ positive neurons in the cortex of the cholesterol-fed rabbits. Furthermore, learning was significantly greater in the higher concentration cholesterol-fed rabbits over controls. The data provide support for our previous finding that dietary cholesterol may facilitate learning and memory.
\end{abstract}




\section{Introduction}

Cholesterol is essential in the CNS and has been shown to play a role in the formation of new synapses as well as synaptic plasticity (Goritz et al., 2002; Koudinov \& Koudinova, 2001; Mauch et al., 2001; Sooksawate \& Simmonds, 2001a; Sooksawate \& Simmonds, 2001b). There is a considerable body of animal data suggesting that a diet high in cholesterol may improve learning and memory. Despite segregation between the peripheral and central cholesterol pools by the BBB (Dietschy \& Turley, 2001), cholesterol metabolites including 24S-hydroxycholesterol and 27-hydroxycholesterol (both permeable to the BBB) allow cross-talk between these pools (Bjorkhem \& Meaney, 2004; Dietschy \& Turley, 2004).

Equally as important; however, cholesterol is involved in atherosclerosis and has been demonstrated to play a role in AD (Schreurs et al., 2003; Sparks et al., 2000b; Sparks \& Schreurs, 2003). The cholesterol-fed rabbit has been used as a model for AD because it shows pathologies consistent with many different features of the AD brain such as neuronal accumulation of A $\beta$ immunoreactivity (Sparks et al., 1994; Sparks, 1996; Sparks \& Schreurs, 2003; Woodruff-Pak et al., 2007), extracellular A $\beta$ plaques (Sparks, 1997b), elevated neuronal and brain cholesterol (Ghribi et al., 2006b; Sparks, 1997b), among others. However, it appears that the cholesterol-fed rabbit model only causes a decrease in learning in the presences of senile plaques. In the absence of plaques, cholesterol has a positive effect on learning and memory. It is currently unknown what causes this difference in effect. Copper, among other trace metals, is essential in the CNS and has been demonstrated to play a crucial role in a number of neuronal functions 
including neurotransmission. At the same time; however, imbalances in CNS copper concentrations play a role in several neurological disorders, including AD. In recent years we have been trying to better understand the relationship copper plays in a cholesterolfed rabbit model. Our findings thus far have warranted further investigation. In a previous paper it was reported that a $2 \%$ cholesterol diet alone is able to have a positive effect on learning and memory and facilitate classical conditioning (Schreurs et al., 2003). However in another publication it was reported that a $2 \%$ cholesterol diet with the addition of $0.12 \mathrm{ppm}$ copper has a detrimental effect on learning and memory (Sparks \& Schreurs, 2003). It is known that cholesterol increases levels of $A \beta$, and $A \beta$ plays a neuroprotective role in the CNS as a metal chelator of redox active metals such as copper. It is currently not known how sensitive the concentration level of cholesterol in the diet is in relation to having a positive or negative effect on learning and memory. To examine the effects of cholesterol concentrations on copper supplementation, we tested the hypothesis that increasing the cholesterol concentration will increase learning and $\mathrm{A} \beta$ immunoreactivity.

We placed four groups of rabbits on an eight-week cholesterol diet, each receiving one of four $(0,0.5,1$, or $2 \%)$ cholesterol-supplemented diets, along with all the groups exposed to an equal amount of copper supplemented drinking water $(0.12 \mathrm{ppm})$. A number of behavioral tests were subsequently employed to assess the effects on learning and sensory processing. The tests included a difficult learning task, transfer to an easy learning task, and a test of sensitivity to the tone. 


\section{Methods}

Subjects: Twenty four male, New Zealand white rabbits (Oryctolagus cuniculus) supplied by Harlan (Indianapolis, IN) weighed approximately 2.0-2.2 kg upon arrival. Animals were housed in individual cages, given free access to food and ultra pure water (18 $\mathrm{M} \Omega$, Millipore Academic) and maintained on a 12-hour light/dark cycle. Rabbits were assigned to four groups ( $\mathrm{n}$ 's $=6$ ) comprising the concentration of cholesterol added to their food: $0,0.5,1$, or $2 \%$. One rabbit from the $1 \%$ group showed excessively low levels of responding to air puff and was excluded from behavioral assessment and only used for histological analysis.

Cholesterol-fed rabbits received $0.5,1$ or $2 \%$ cholesterol incorporated into Purina rabbit chow (Purina 5321; Dyets Inc., Bethlehem, PA) for 8 weeks prior to behavioral experiments, and normal chow control rabbits $(0 \%$ cholesterol $)$ received standard Purina rabbit chow (Purina 5321, 0\% added cholesterol). Cholesterol was supplied as lanolin that was dissolved in ether and mixed with Purina 5321 rabbit chow meal. The ether was allowed to evaporate, and the meal was then formed into pellets (Alissa et al., 2004). Numerous reports have shown that rabbits fed a $2 \%$ cholesterol diet for eight weeks have serum cholesterol levels approaching 2,000 mg/dl whereas a 1\% cholesterol diet results in levels of approximately $1,400 \mathrm{mg} / \mathrm{dl}$ and a $0.5 \%$ cholesterol diet results in levels of 700 $\mathrm{mg} / \mathrm{dl}$ compared to rabbits fed normal chow that have levels of less than $40 \mathrm{mg} / \mathrm{dl}$ (Henry \& Bentley, 1981; Huang et al., 2004b; Lamb et al., 2001; Sparks, 1997b). Although rabbits on the cholesterol diets did not gain as much weight as controls (see Results), they continued to eat the same amounts of food (100-150 g/day). All rabbits had drinking 
water supplemented with 0.12 parts per million ( $\mathrm{ppm}$ ) copper as copper sulfate previously shown to increase the number of $A \beta$ labeled cells in the brain (Sparks et al., 2006b). Rabbits were maintained in accordance with guidelines issued by the National Institutes of Health and the research was approved by the West Virginia University Animal Care and Use Committee.

Apparatus: The apparatus has been detailed by Schreurs and Alkon (1990) who modeled their apparatus after those described by Gormezano (Coleman \& Gormezano, 1971; Gormezano, 1966). Each rabbit was restrained in a Plexiglas box and trained in a soundattenuating, ventilated chamber (Coulbourn Instruments, Allentown, PA; Model E10-20). A stimulus panel containing a speaker and a house light (10-W, 120-V incandescent lamp) was mounted at a $45^{\circ}$ angle, $15 \mathrm{~cm}$ anterior to and $15 \mathrm{~cm}$ above the subject's head. An ambient noise level of $65 \mathrm{~dB}$ was provided by an exhaust fan. A programmable air pressure delivery system (Model ER-3000, Tescom Corp., Elk River, MN) was used to deliver a puff of air through a tube ( $1 \mathrm{~mm}$ internal diameter) positioned $5 \mathrm{~mm}$ from and perpendicular to the center of the cornea.

Details of transducing nictitating membrane (NM) movements have been reported previously (Gormezano \& Gibbs, 1988; Schreurs \& Alkon, 1990). A 1-mm hook connected to an L-shaped lever containing a freely moving ball and socket joint was attached to a 6-0 nylon loop sutured into, but not through, the NM. The other end of the lever was attached to a potentiometer (Novotechnik US Inc., Southborough, MA; Model P2201) that, in turn, was connected to a 12-bit analog-to-digital converter (5-ms sampling rate; $0.05-\mathrm{mm}$ resolution). Individual analog-to-digital outputs were stored on a trial-by- 
trial basis for subsequent analysis. Data collection, analysis and stimulus delivery were accomplished using a LabVIEW system (National Instruments, Austin, TX).

NMR classical conditioning: All rabbits received one day of adaptation, eight daily sessions of tone and air puff trace conditioning, four daily sessions of tone and air puff delay conditioning and two days of tone intensity testing. Adaptation was designed to habituate the rabbits to restraint and the training chambers. Trace conditioning provided an opportunity to assess the effects of cholesterol concentration on the acquisition of a difficult learning task and delay conditioning enabled us to ensure that rabbits could all learn a relatively simple conditioning task. Finally, tone intensity testing allowed us to determine whether the cholesterol diets had any effects on the rabbits' ability to hear. Following four days of delay conditioning, all rabbits were responding at a reliable level to the tone CS and we probed their hearing by pairing tones that were softer and louder than the training intensity $(82 \mathrm{~dB})$ with the air puff.

On adaptation day, the rabbits were prepared for recording of NM movement and then adapted to the training chambers for the length of time of subsequent training sessions (60 min). Each of the eight paired trace conditioning sessions consisted of 60 presentations of a $100-\mathrm{ms}, 1-\mathrm{KHz}, 82-\mathrm{dB}$, tone CS that was followed by a $500-\mathrm{ms}$ trace interval and then a 100-ms, 4-psi air puff US (i.e., 600-ms interstimulus interval). Each of the four days of paired delay conditioning consisted of 60 presentations of a 400-ms, 1-KHz, 82-dB, tone CS that coterminated with a 100-ms, 4-psi air puff US (i.e., 300-ms interstimulus interval). Stimulus presentations were delivered, on average, every $60 \mathrm{~s}$ (50-70 $\mathrm{s}$ range). The two tone intensity testing sessions consisted of the presentation of 
one of seven $100-\mathrm{ms}$ tone intensities $(60,65,70,75,80,85,90 \mathrm{~dB})$ or a zero intensity $(0$ dB) followed by a 200-ms trace interval and then a 100-ms, 4-psi air puff US. Each tone intensity-air puff pairing was presented eight times as a randomized sequence with a trial

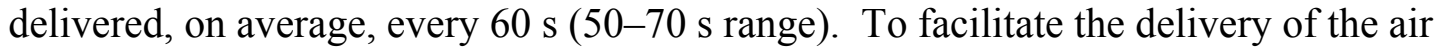
puff US, the eyelids were loosely held open during each session with tailor's hooks attached to an adjustable elastic strap (Buck et al., 2001).

A CR was defined as any extension of the NM exceeding $0.5 \mathrm{~mm}$ that was initiated after CS onset but prior to US onset (Schreurs et al., 2000).

Histology: In order to determine the effects of the cholesterol diet on the brain, rabbits were anesthetized deeply with a cocktail of Ketamine $(640 \mathrm{mg} / \mathrm{kg}) /$ Xylazine $(130$ $\mathrm{mg} / \mathrm{kg}$ ) and perfused transcardially with $0.9 \%$ saline. Brains were extracted and postfixed for fourteen days in $4 \%$ paraformaldehyde. Fifty-micron vibratome sections of the hippocampus and surrounding cortex were immunostained with an antibody to $A \beta$ (10D5, 1:100 dilution; provided by Dr. Dale Schenk of Elan Pharmaceuticals) using published peroxidase-antiperoxidase immunohistochemical methods (Sparks et al., 1994). The cells positively stained for the 10D5 antibody within a $0.5 \times 0.5 \mathrm{~mm}$ square grid were counted in at least eight randomly selected areas of the cortex and at least four areas of the hippocampus within a randomly selected section using a $20 \mathrm{X}$ objective as previously described (Schreurs et al., 2003; Sparks et al., 2002; Sparks et al., 2006b; Sparks \& Schreurs, 2003). Counts were made by a researcher who was blind to the treatment conditions (G.S.). 
Copper levels: Copper levels in serum were measured using the Association of Official Analytical Chemists (AOAC) Method 983.24 - atomic absorption spectrophotometric method for copper in serum with the modification that samples were diluted $(1+4)$ in water rather than $(1+1)$. Copper levels in brain were measured using an adaptation of AOAC Method 985.40 - atomic absorption spectrophotometric method for copper in liver with the modification that samples were diluted $(1+9)$ instead of $(1+24)$.

Statistical analysis: Analysis of variance was used to test for main effects and interactions and post hoc comparisons between groups were based on statistical tests of contrasts between group means (Bird, 2004). 


\section{Results}

Body weight. Figure 4.1 shows and analysis of rabbit weights confirmed that after eight weeks on the diet, rabbits on the $0.5 \%, 1 \%$, and $2 \%$ cholesterol diets had gained less weight $(2.82 \mathrm{~kg})$ than the $0 \%$ cholesterol control group $(3.44 \mathrm{~kg}, \mathrm{p}<.001)$. There were no differences in the weights of rabbits on the $0.5 \%, 1 \%$, and $2 \%$ cholesterol diets.

Behavior. Figure 4.2 shows mean percent CRs for rabbits in the four cholesterol groups across the eight days of trace conditioning and the four days of delay conditioning. The figure shows very clear differences in the acquisition of NMR conditioning between the four groups as a function of days of trace conditioning. The $2 \%$ cholesterol group showed higher levels of responding than the remaining groups and the $1 \%$ cholesterol group reached a comparable terminal level of responding to the $2 \%$ group at the end of the trace conditioning phase. Analysis of mean percent CRs during trace conditioning

yielded a significant main effect of cholesterol concentration $[F(3,19)=3.91, p<.05]$, a significant effects of days $[F(7,133)=20.67, p<.001]$, and a significant interaction of cholesterol concentration by days $[\mathrm{F}(21,133)=3.14, \mathrm{p}<.001]$. Post hoc orthogonal contrasts (Bird, 2004) confirmed that the $2 \%$ group was significantly higher than the other three groups across the eight days of trace conditioning $(\mathrm{p}<.01)$, and that the $1 \%$ group was different from the $0.5 \%$ and $0 \%$ groups $(\mathrm{p}<.05)$ which, in turn, were not different from each other.

Rabbits were then shifted to delay conditioning and all groups showed rapid acquisition of CRs to levels at or near $90 \%$. The relationship among the groups from the 
trace conditioning phase was retained during delay conditioning with the $2 \%$ and $1 \%$ cholesterol groups responding at higher levels than the $0.5 \%$ and $0 \%$ cholesterol groups. Analysis of mean percent CRs during delay conditioning yielded a significant effects of days $[F(3,57)=84.86, p<.001]$ and an interaction of cholesterol concentration by days $[F(9,57)=2 . .07, p<.05]$. Post hoc contrasts confirmed that the $2 \%$ and $1 \%$ cholesterol groups showed higher levels of response acquisition than the $0.5 \%$ and $0 \%$ groups $(\mathrm{p}<$ .05) which, in turn, were not different from each other.

Tone Intensity. Figure 4.3 shows mean percent CRs to eight intensities of the tone conditioned stimulus for rabbits in the $0,0.5,1$ and $2 \%$ cholesterol groups collapsed across two additional days of trace conditioning. The figure shows that the level of responding was a function of tone intensity and that all groups responded at about the same levels. Despite some suggestion in the figure that the $0.5 \%$ cholesterol group may have responded less at the higher tone intensities, analysis only yielded a significant main effect of tone intensity $[\mathrm{F}(7,133)=106.32, \mathrm{p}<.001]$. There were no significant effects of cholesterol concentration even when each of the tone intensities was analyzed separately (all F's < 1).

Histology. Figure 4.4 shows mean number and Figure 4.5 shows low-power photomicrograph examples of $A \beta$ immunoreactive (labeled) cells in the cortex and hippocampus. Examination of Figure 4.4 shows the number of $A \beta$-labeled cells in the cortex and, to a lesser extent in the hippocampus, was a function of the cholesterol concentration. Figure 4.5 shows there was no evidence of extracellular plaque-like 
structures in the cortex or hippocampus at any cholesterol concentration. Analysis of the number of $A \beta$ labeled cells in the cortex revealed a significant effect of cholesterol concentration $[\mathrm{F}(3,20)=77.14, \mathrm{p}<.001]$ and post hoc comparisons of the number of labeled cells in the cortex revealed that they were a function of the cholesterol concentration. The smallest difference between groups in the number of labeled cells was between the $0 \%$ and $0.5 \%$ cholesterol groups $(3.35 \pm 0.25$ vs. $5.03 \pm 0.44, p<.05)$. Analysis of the number of $A \beta$ labeled cells in the hippocampus also revealed a significant effect of cholesterol concentration $[\mathrm{F}(3,20)=16.62, \mathrm{p}<.001]$ but in this case post hoc comparisons revealed that only the $2 \%$ cholesterol group had significantly higher numbers of $A \beta$-labeled cells than the remaining groups $(p<.001)$. This significantly higher number of $\mathrm{A} \beta$-labeled cells in the hippocampus is consistent with the higher overall level of trace conditioning in the $2 \%$ cholesterol group and with the essential role of the hippocampus in trace conditioning.

Copper Levels. An analysis of copper levels in serum and brain as a result of $0.12 \mathrm{ppm}$ copper in the drinking water revealed no significant differences between the groups in either serum or brain copper levels (F's $<1.8$ ). 


\section{Discussion}

The principal findings of the current experiment were that the level of classical conditioning and the numbers of $\mathrm{A} \beta$-labeled cells were all a function of cholesterol concentration. We have previously shown that cholesterol can enhance NMR conditioning (Schreurs et al., 2003), and the present results extend this finding to show that the level of facilitation is a function of cholesterol concentration. In addition to the

present results, a recent report shows that feeding cholesterol to young, normal rats also improves learning - in this case spatial learning and memory in the Morris water maze (Dufour et al., 2006). Taken together, the results from a number of different species including transgenic mice, normal and cholesterol-deficient rats and normal rabbits subjected to a number of different behavioral paradigms including spatial learning and classical conditioning suggest that dietary cholesterol may improve learning and memory.

Given that dietary cholesterol does not cross the BBB directly in any appreciable amount (Bjorkhem \& Meaney, 2004; Dietschy \& Turley, 2001; Dietschy \& Turley, 2004; Tuma \& Hubbard, 2003), it remains to determine how trace conditioning, which is controlled in part by the hippocampus, was facilitated by a cholesterol-rich diet. The same is true for the effects of cholesterol on rat water maze learning (Dufour et al., 2006) and previous research showing that addition of cholesterol to the diet of animals deficient in cholesterol or that have cholesterol synthesis blocked reverses learning and memory deficits (Endo et al., 1996; O'Brien et al., 2002; Voikar et al., 2002; Xu et al., 1998).

One possible explanation for the facilitative effects of dietary cholesterol on the brain is that cholesterol metabolites that do cross the BBB, particularly 27 - 
hydroxcholesterol, may have had some influence on learning (Heverin et al., 2005). There is evidence, albeit indirect, for the involvement of 27-hydroxycholesterol in learning and memory because levels of this oxysterol are significantly reduced in patients with mild cognitive impairment, AD and vascular dementia (Kolsch et al., 2004).

Another possible explanation is the effect of dietary cholesterol on the number of $A \beta$-labeled cells in the brain. The significant increase in the number of $A \beta$ immunoreactive neurons as a function of cholesterol concentration particularly in the hippocampus in the $2 \%$ cholesterol group, suggests a role for intracellular $\mathrm{A} \beta$ in learning and memory. Indeed, there is good evidence for the normal function of $A \beta$ in cellular processes (Pearson \& Peers, 2006; Plant et al., 2003) and in fact, A $\beta$ modulates potassium channels (Plant et al., 2006) - an important mediator of many forms of learning and memory (Giese et al., 1998; Nelson et al., 1999; Schrader et al., 2002; Schreurs et al., 1998). It is certainly possible that by increasing the number of $A \beta$ labeled neurons, the cholesterol diet facilitated NMR conditioning by modulating cellular potassium channels and affecting neuronal excitability - a mechanism known to be involved in learning and memory (Johnston et al., 2003; Xu \& Kang, 2005; Zhang \& Linden, 2003). The significantly higher levels of trace conditioning and higher numbers of $\mathrm{A} \beta$-labeled neurons in the hippocampus (a structure essential for trace conditioning) for the $2 \%$ cholesterol group are consistent with such a hypothesis.

The highly conserved LDLRs are yet another potential mechanism by which cholesterol can affect learning and memory (Herz \& Chen, 2006; Qiu et al., 2005). These receptors are known to regulate cholesterol transport and are capable of transducing a number of extracellular signals across the BBB and may play a role in synaptic plasticity 
via NMDA receptors (Herz \& Chen, 2006). Perhaps the most well known of the LDLR ligands is ApoE - a protein known to be implicated in $\mathrm{AD}$. Recent research suggests that this class of receptors may be involved in learning and memory because a transgenic mouse model of AD lacking the LDLR developed larger spatial learning deficits than a transgenic mice with the receptor (Cao et al., 2006).

A final possibility is that pro-inflammatory cytokines including interleukin $1 \alpha$ and $1 \beta$, tumor necrosis factor $\alpha$, and interleukin- 6 known to be induced by a high cholesterol diet (Fazio \& Linton, 2001; Kaul, 2001; Rahman et al., 2005), cross the BBB to affect learning and memory. The role of cytokines in learning and memory has been explored by a number of groups most notably Maier and colleagues who have found that interleukin $1 \beta$ can affect fear conditioning (Barrientos et al., 2006; Maier \& Watkins, 2003; Thomson \& Sutherland, 2005). Specifically, it was found that interleukin $1 \beta$ interferes with the consolidation of hippocampally-dependent contextual fear conditioning (Maier \& Watkins, 2003). Using a T-maze avoidance paradigm, Banks and colleagues found that interleukin $1 \alpha$ crossed the BBB to impair memory. On the other hand, Brennan and colleagues have found evidence that the pro-inflammatory cytokine tumor necrosis factor $\alpha$ facilitated performance of avoidance learning (Brennan et al., 2004; Brennan \& Tieder, 2006). More important for the present results, is the finding that systemic injections of interleukin $1 \beta$ facilitate classical eye blink conditioning in male rats (Servatius \& Beck, 2003). Consequently, it is possible that a high cholesterol diet may facilitate learning via pro-inflammatory cytokines.

Taken together, the results of the present experiment as well as other animal experiments suggest that cholesterol may facilitate learning and memory. However, there 
is also a considerable body of evidence from animal experiments on the negative effects of cholesterol on learning and memory. For example, decreasing cholesterol in aged animals improves learning in the Morris water maze (Kessler et al., 1986; Yehuda et al., 1998; Yehuda \& Carasso, 1993). Similarly, rats, mice and rabbits given calcium channel blockers that, among other things, decrease the level of cholesterol by reducing the estrification of cholesterol and increasing hydrolysis of existing cholesterol esters (Nayler, 1999; Schachter, 1997), demonstrate improvements in learning and memory in a number of tasks including the Morris water maze and classical conditioning of the rabbit NMR (Deyo et al., 1989; Kane \& Robinson, 1999; Quartermain, 2000; Woodruff-Pak et al., 1997).

In addition to differences among animal studies on the effects of cholesterol on learning and memory, human studies also appear to be mixed. On one hand, recent studies show that language (Reitz et al., 2005) and cognitive performance (Elias et al., 2005) are a function of increasing plasma lipid levels in the healthy elderly. On the other hand, a larger number of studies show that elevated serum cholesterol is a significant risk factor for mild cognitive impairment (Kivipelto et al., 2001; Näslund et al., 2000; Yaffe et al., 2002) and AD (Evans et al., 2000; Hartmann, 2001; Papassotiropoulos et al., 2005; Poirier, 2005; Schonknecht et al., 2002; Simons et al., 2001; Sparks et al., 1990; Whitmer et al., 2005; Wood et al., 2005; Yanagisawa, 2003) and that cholesterol levels may be negatively correlated with measures of intelligence (Atzmon et al., 2002; Muldoon et al., 1997; Reitan \& Shipley, 1963; van Exel et al., 2002; Yaffe et al., 2002). Moreover, epidemiological evidence is mixed on the relationship between cholesterol levels and the cognitive deficits characteristic of AD (Evans et al., 2000; Hartmann, 2001; Jarvik et al., 
1995; Notkola et al., 1998; Simons et al., 2001; Stewart et al., 2001; Wood et al., 2005).

Finally, there is controversy about whether lowering cholesterol with statins may ameliorate some of the cognitive symptoms of AD (Shobab et al., 2005). For example, some retrospective epidemiological studies have suggested that statin treatment has an effect on AD (Rockwood et al., 2002; Wolozin et al., 2000) as have some prospective studies (Simons et al., 2002b; Sparks et al., 2006a) whereas others have not (Rockwood, 2006). In a recent animal study, lowering cholesterol with a statin produced improvements in learning and memory independently of $\mathrm{A} \beta$ levels in the brain (Li et al., 2006). Specifically, Li et al. (2006) found that simvastatin reversed spatial maze learning deficits not only in a transgenic mouse model of AD that showed high levels of $A \beta$ but also improved spatial maze learning in their non-transgenic littermates that did not have high levels of $\mathrm{A} \beta$.

In conclusion, it is almost certainly true that the negative health effects of high cholesterol outweigh its potential positive effects on learning and memory. However, what remains to be determined is how, despite the severe pathophysiology caused by a high-cholesterol diet including atherosclerosis, coronary heart disease and liver dysfunction (Sparks, 1997a), many cholesterol-fed animals learn better than normal controls. One answer may lie in the separation of the peripheral and central cholesterol pools. Dietary cholesterol may have a negative effect on the body but an indirectly positive effect on the brain via cholesterol metabolites, increased neuronal A $\beta$, the LDL receptor, or pro-inflammatory cytokines. There are a number of other examples where high cholesterol damages the body but still has positive effects on the brain. For example, dietary cholesterol has been shown to provide protection from seizures 
(Alexander \& Kopeloff, 1971), hypoxia (Xi et al., 2006) and stroke (Vauthey et al., 2000; Zuliani et al., 2004). In fact, a recent study shows that although total serum cholesterol is positively correlated with coronary heart disease, it is negatively correlated with stroke (Cui et al., 2006). Low serum cholesterol levels have also been associated with mood disorders and suicide (Beaslely et al., 2005). Nevertheless, at some point, a high cholesterol diet begins to have deleterious effects on the brain $-\mathrm{A} \beta$ plaque-like structures form and learning and memory are debilitated (Sparks \& Schreurs, 2003). Clearly, we need to eliminate the negative effects of high cholesterol while determining the nature of its positive effects on learning and memory. 


\section{Figure 4.1}

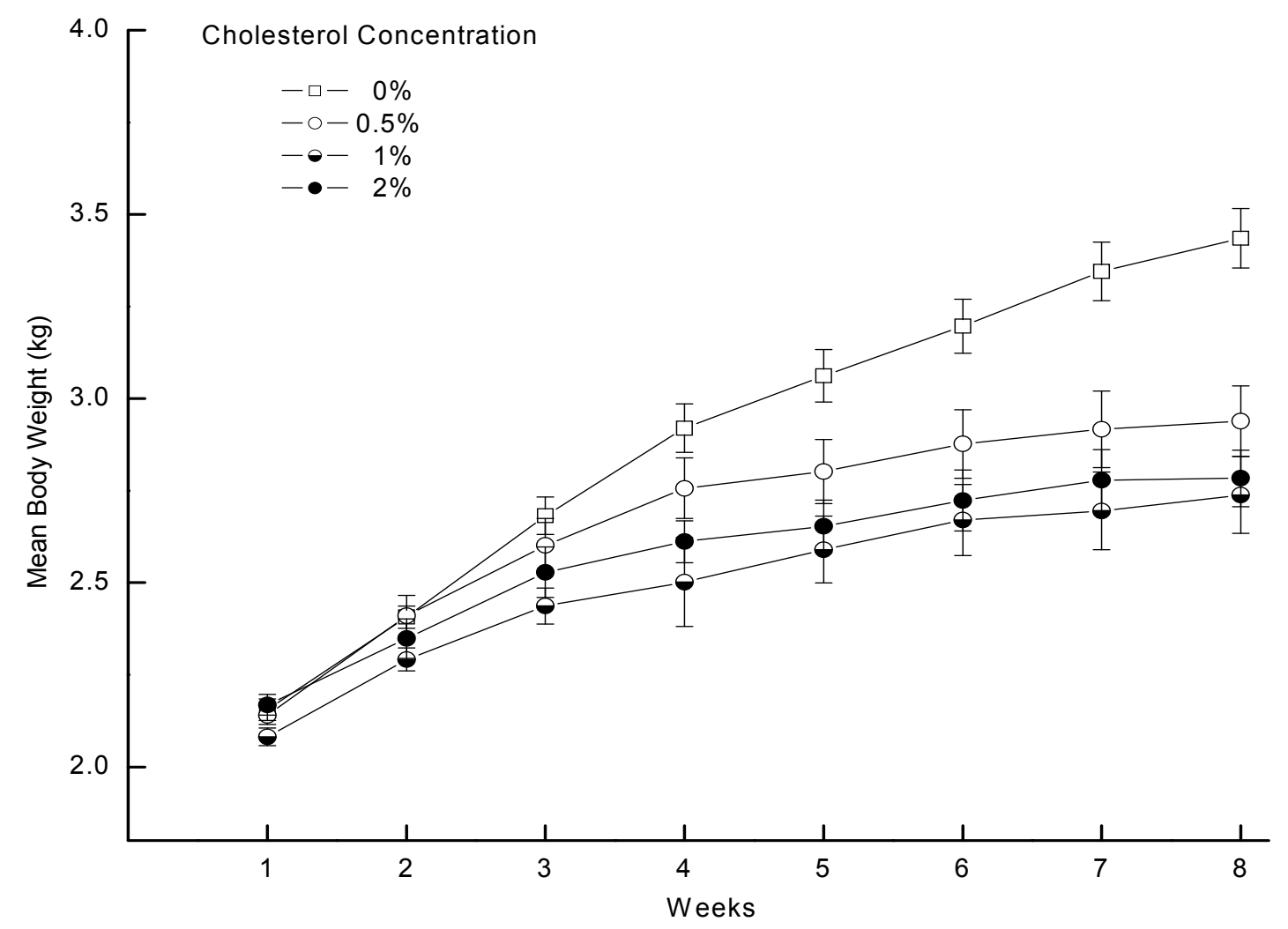

Figure 4.1. Mean body weight for rabbits. This illustrates the weights of the $0 \%, 0.5 \%$, $1 \%$ and $2 \%$ cholesterol groups across the eight weeks of the cholesterol diet. 


\section{Figure 4.2}

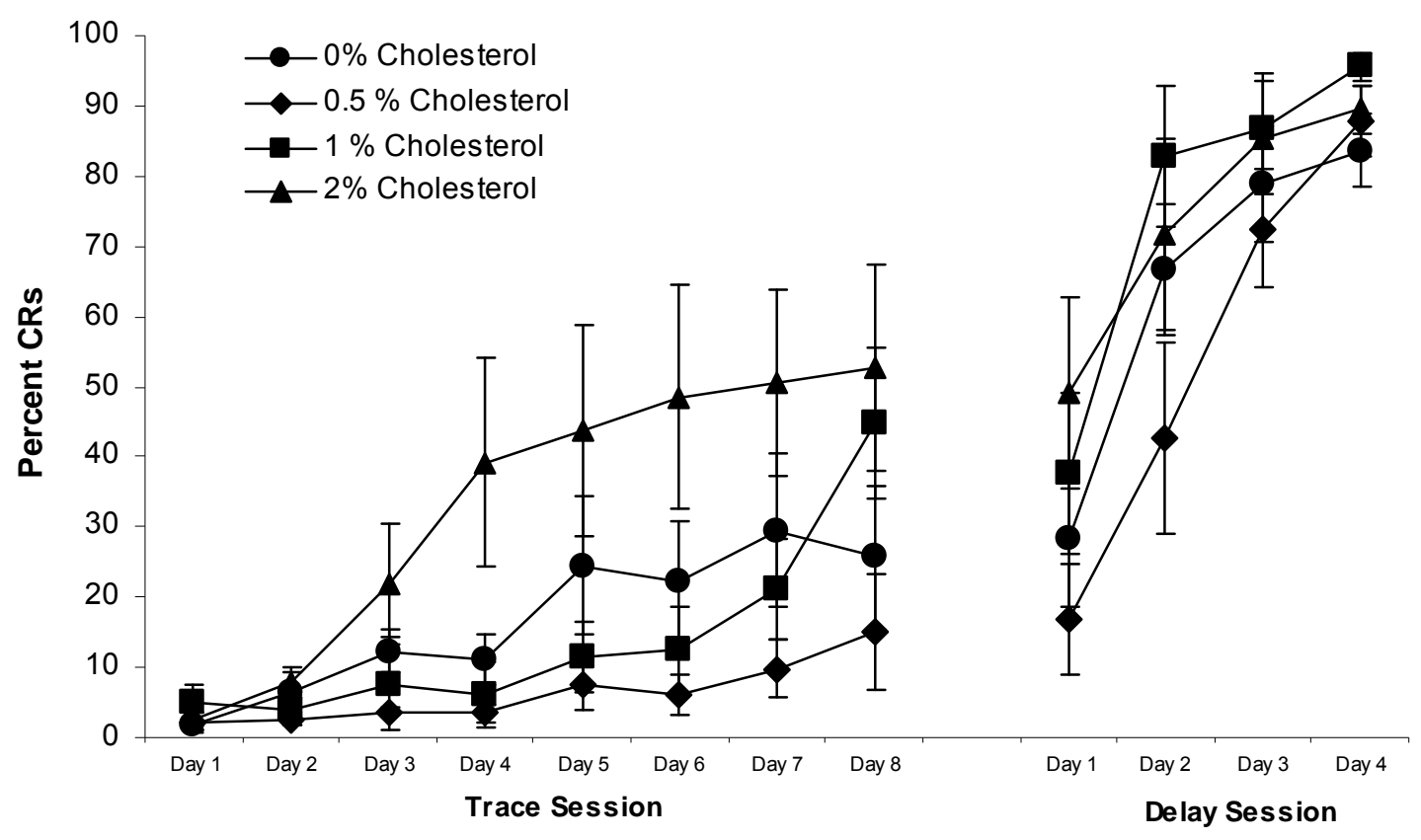

Figure 4.2. Mean conditioning responses of rabbits. This illustrates the mean percent conditioned nictitating membrane responding (CRs) to the conditioned stimulus (CS) for rabbits in the $0 \%, 0.5 \%, 1 \%$ and $2 \%$ cholesterol groups across the eight days of trace conditioning and the four days of delay conditioning. 


\section{Figure 4.3}

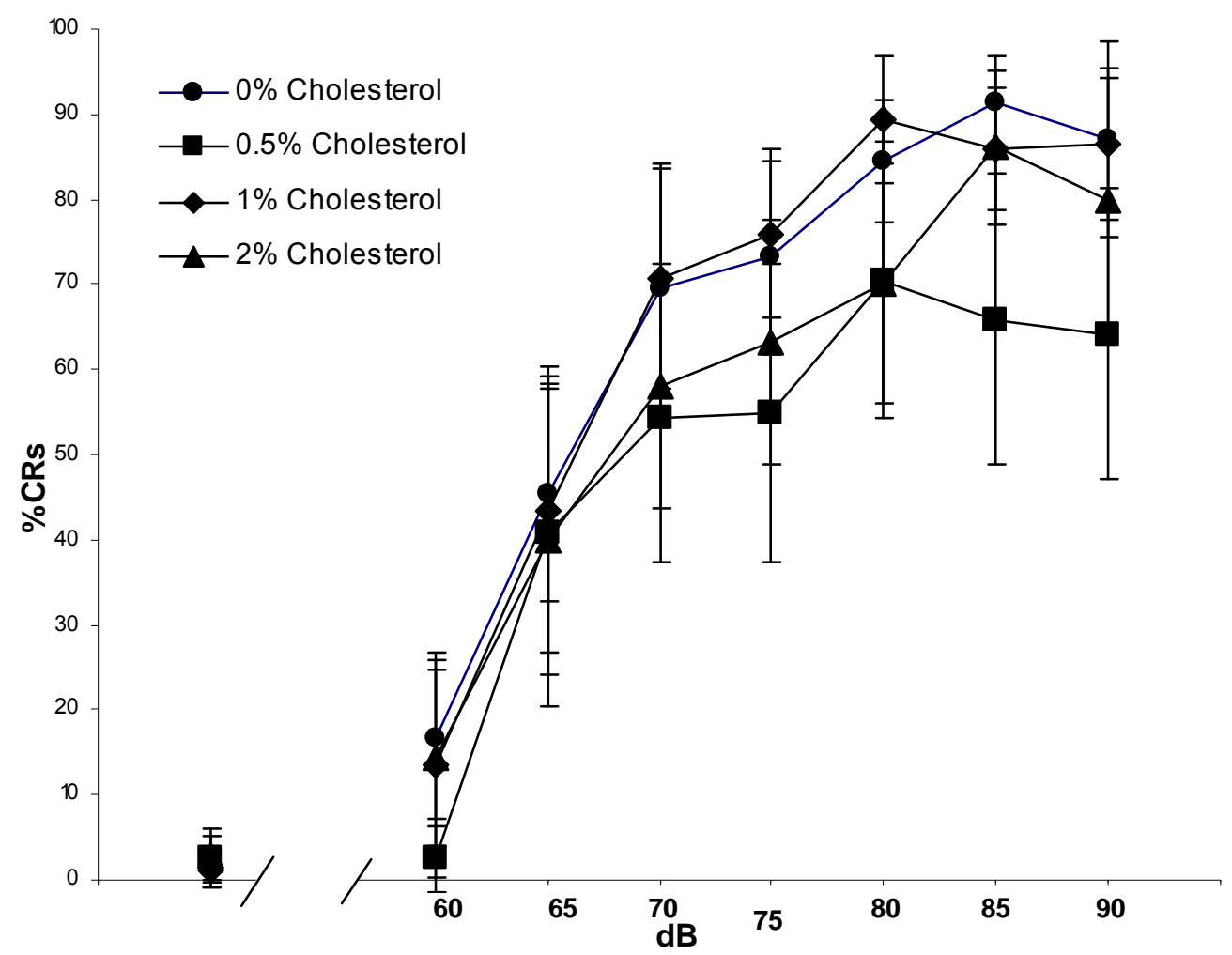

Figure 4.3. Tone intensity testing. Mean percent conditioned nictitating membrane responding (CRs) to eight intensities of the tone conditioned stimulus (CS; 0, 60, 65, 70, $75,80,85$ and $90 \mathrm{~dB}$ ) for rabbits in the $0 \%, 0.5 \%, 1 \%$ and $2 \%$ cholesterol groups collapsed across two days of tone intensity presentations following delay conditioning. 


\section{Figure 4.4}

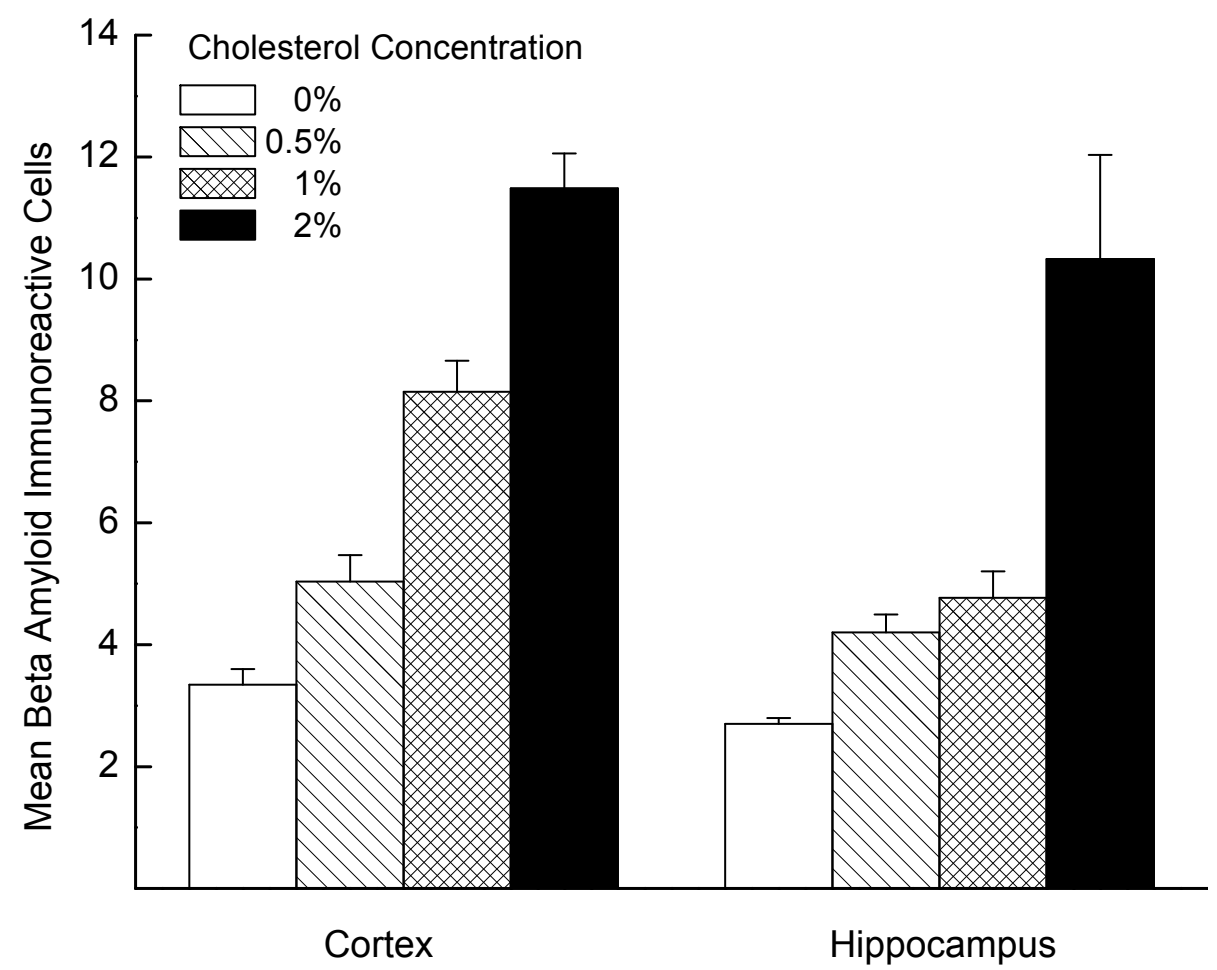

Figure 4.4. Mean number A $\beta$ immunoreactive (labeled) cells. Cells were labeled in the cortex and hippocampus of rabbits in the $0 \%, 0.5 \%, 1 \%$ and $2 \%$ cholesterol groups. Fifty-micron vibratome sections of the hippocampus and surrounding cortex were immunostained with an antibody to $\mathrm{A} \beta$ (10D5, 1:100 dilution; provided by Dr. Dale Schenk of Elan Pharmaceuticals). Cells positively stained for the10D5 antibody within a $0.5 \times 0.5 \mathrm{~mm}$ square grid were counted in at least eight randomly selected areas in the cortex and four randomly selected areas of the hippocampus within a randomly selected section using a 20X objective. Counts were made by a researcher (GS) who was blind to the treatment conditions. 


\section{Figure 4.5}
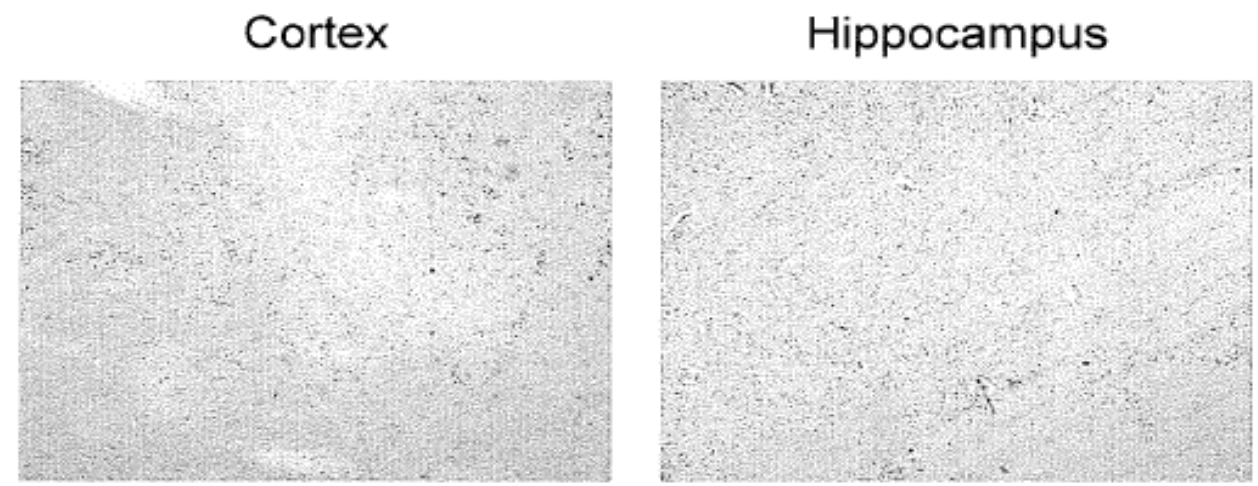

$0 \%$
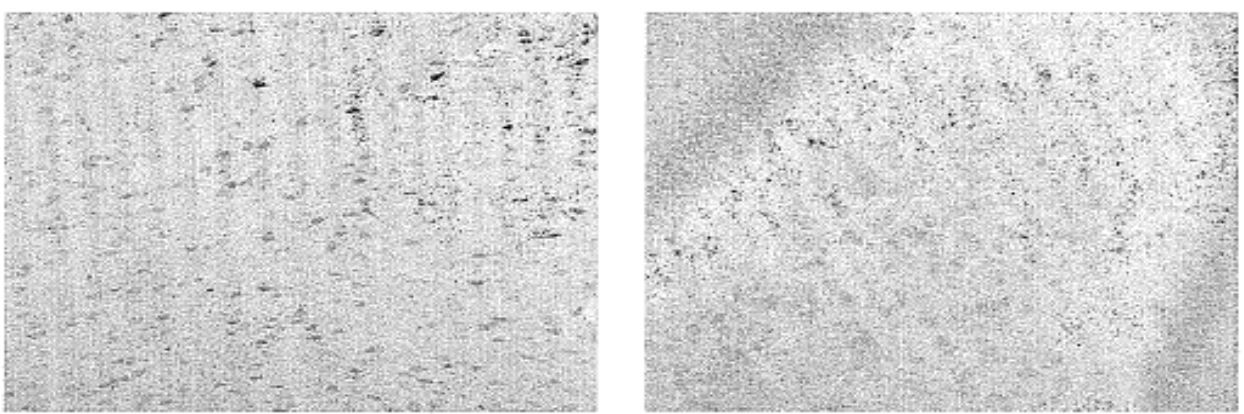

$0.5 \%$
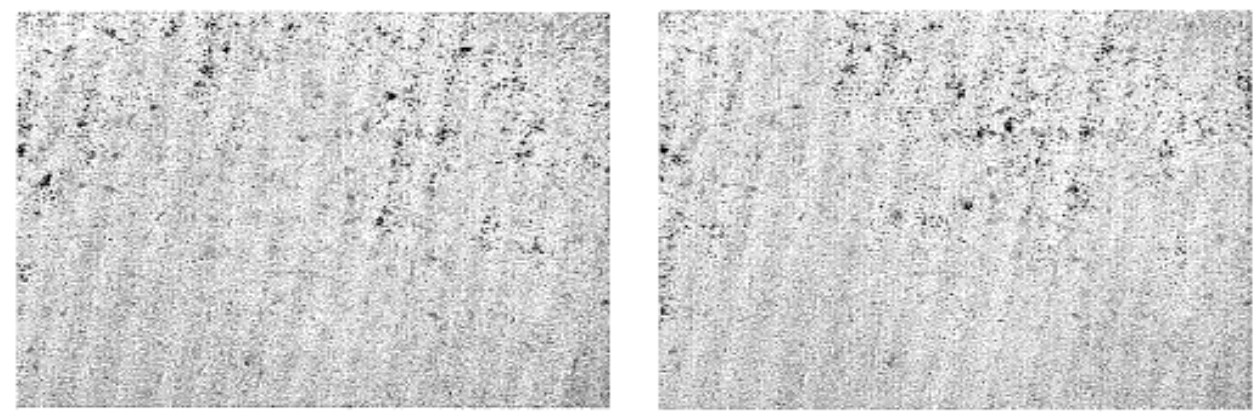

$1 \%$
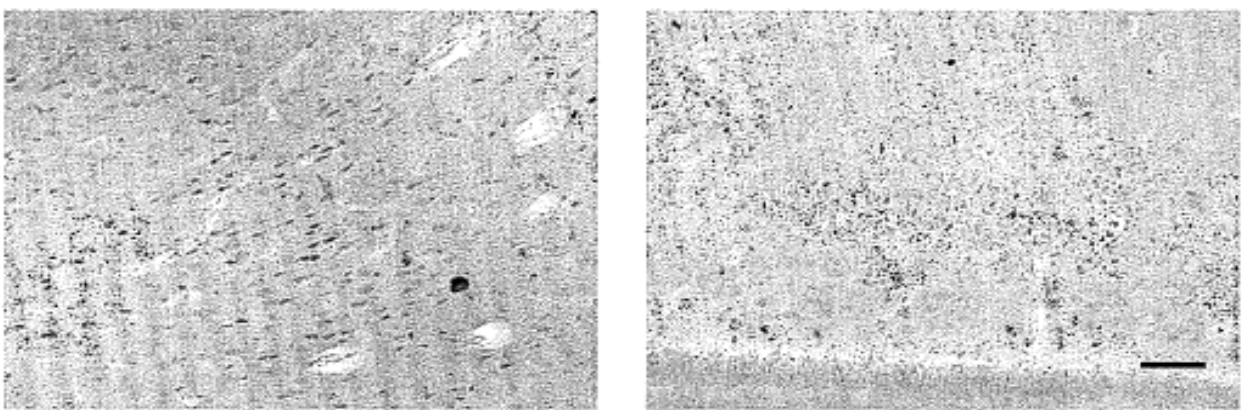

$2 \%$

Figure 4.5. Low power photomicrographs of representative $A \beta$ immunoreactive

(labeled) cells. Cells in the cortex (left) and hippocampus (right) of a rabbit from the $0 \%$, $0.5 \%, 1 \%$ and $2 \%$ cholesterol groups. Scale bar $100 \mu \mathrm{m}$. 
Chapter Five

Dietary Cholesterol Impairs Memory and Memory Increases Brain Cholesterol and Sulfatide Levels

This work is published in Behavioral Neuroscience 124: 115-123 (2010) 


\begin{abstract}
Cholesterol and sulfatides play many important roles in learning and memory. To date, our observations about the effects of cholesterol on learning have been assessed during response acquisition i.e., the learning of a new memory. Here we report for the first time on the effect of a cholesterol diet on a previously formed memory. Rabbits were given trace conditioning of the NMR for ten days, then fed a $2 \%$ cholesterol diet for eight weeks, and then assessed for memory recall of the initially learned task. We show that dietary cholesterol had an adverse effect on memory recall. Second, we investigated whether dietary cholesterol caused an increase in brain cholesterol and sulfatide levels in four major brain structures (hippocampus, frontal lobe, brainstem, and cerebellum) using a technique for analyzing myelin and myelin-free fractions separately. Although our data confirm previous findings that dietary cholesterol does not directly affect cholesterol and establish that it does not affect sulfatide levels in the brain, these levels did increase rather significantly in the hippocampus and frontal lobe as a function of learning and memory.
\end{abstract}




\section{Introduction}

The effect of dietary cholesterol on learning and memory remains unclear. On the one hand, a significant body of research has demonstrated a positive effect of dietary cholesterol on learning in several animal species including rats, mice, and rabbits (Dufour et al., 2006; Miller \& Wehner, 1994; Schreurs et al., 2003; Upchurch \& Wehner, 1988; Voikar et al., 2002). Feeding cholesterol to rats that are either deficient in cholesterol or have cholesterol synthesis suppressed ameliorates learning and memory impairments (Endo et al., 1996; O'Brien et al., 2000; Voikar et al., 2002; Xu et al., 1998). Dufour and colleagues (2006) have shown feeding rats $2 \%$ cholesterol enhances water maze learning. Our recent studies have also consistently shown an increase in the level of rabbit classical conditioning following an 8-week 2\% cholesterol diet (Schreurs et al., 2003; Schreurs et al., 2007b). On the other hand, there is evidence that dietary cholesterol can have a negative effect on learning and memory (Kessler et al., 1986; Yehuda et al., 1998; Yehuda \& Carasso, 1993). In a recent study, feeding mice a 2\% cholesterol diet for 8 weeks resulted in loss of working memory (Thirumangalakudi et al., 2008). Moreover, reducing cholesterol levels with statins results in improved memory suggesting cholesterol has a detrimental effect on learning and memory (Li et al., 2006).

It is unknown if a cholesterol diet has any effect on previously formed memories. To date, our observations about the effects of cholesterol on learning have been assessed during response acquisition i.e., the learning of a new memory (Schreurs et al., 2003; Schreurs et al., 2007b). Given that dietary cholesterol plays a significant role in learning new memories, we investigated whether a cholesterol diet might also have an effect on 
previously formed memories (memory recall) using our $2 \%$ cholesterol-fed rabbit model (Schreurs et al., 2003; Schreurs et al., 2007a; Schreurs et al., 2007b; Sparks \& Schreurs, 2003).

To better understand the relationship between dietary cholesterol and learning and memory, it is important to know if dietary cholesterol has any direct effects on brain cholesterol. The relationship between high dietary cholesterol and brain cholesterol still remains unclear and the relationship between high dietary cholesterol and brain sulfatides has not been studied. Both cholesterol and sulfatides serve important roles in the CNS. Cholesterol plays an important role in synapse formation, receptor function, myelin formation and synaptic plasticity (Dietschy \& Turley, 2004; Dufour et al., 2006; Frank et al., 2008; Goritz et al., 2002; Guo et al., 2008; Koudinov \& Koudinova, 2001; Mauch et al., 2001; Saher et al., 2005; Sooksawate \& Simmonds, 2001a). Cholesterol levels in the CNS are tightly controlled and disturbances in cholesterol are correlated with disorders in humans such as Niemann-Pick disease Type C, Smith-Lemli-Opitz syndrome and AD (Ikonen, 2006; Mori et al., 2001; Papassotiropoulos et al., 2002; Voikar et al., 2002; Whitney et al., 2002; Xie et al., 2000). Sulfatides are a class of sulfated galactocerebrosides that have an important role in myelin sheath and axon structure maintenance (Marcus et al., 2006; Ramakrishnan et al., 2007), modulation of potassium and sodium ion channels (Chi \& Qi, 2006; Ishibashi et al., 2002), and learning and memory (D'Hooge et al., 2001). Furthermore, sulfatides have been shown to be altered in a number of neurological disorders including AD (Cheng et al., 2008; Han et al., 2002; Han et al., 2003b; Han, 2007; Zeng \& Han, 2008). 
Some studies have shown feeding animals high levels of cholesterol results in an increase in brain cholesterol levels (Boleman et al., 1998; Dufour et al., 2006;

Schoknecht et al., 1994; Sparks, 1997b). There is only one study, however, that reported a significant increase in the level of cholesterol in the brain of cholesterol-fed rabbits (Sparks, 1997b). Another study found an increase in the cholesterol content of hippocampal neurons using immunofluorescent analysis but chemical analysis of total brain and hippocampus cholesterol levels showed no significant differences from control rabbits (Ghribi et al., 2006b). Finally, a study using cholesterol-fed rats detected differences in hippocampal cholesterol levels after a two-month feeding period but not after a five-month feeding period (Dufour et al., 2006). It is possible that some studies may have been insensitive to subtle changes in brain cholesterol levels of brain structures such as the frontal lobe and the hippocampus that contain smaller amounts of total cholesterol. To date, there is no evidence that a high cholesterol diet has any effect on sulfatide levels in the brain.

The largest amounts of cholesterol and sulfatides in the brain are found in myelin, and recently it has been shown that cholesterol-fed rabbits suffer damage to the myelin sheath (Berkman et al., 2009). Therefore, in this experiment we used a technique for analyzing myelin and myelin-free fractions separately in order to reveal potentially subtle changes in cholesterol and sulfatide levels resulting from the diet. It is also possible that these levels are the same in a high-cholesterol and cholesterol-free diet but that the distribution of cholesterol and sulfatides among brain structures may be different. Given learning and memory are dependent on a number of brain structures (e.g., cerebellum, 
frontal lobe, hippocampus, and brainstem), we investigated the levels and distribution of cholesterol and sulfatides in each of these structures. 


\section{Methods}

Subjects: Subjects were 24 two-month old male New Zealand white rabbits (Oryctolagus cuniculus) weighing approximately $2.0-2.5 \mathrm{~kg}$ at the beginning of the experiment. Animals were housed in individual cages, given free access to food and distilled water, and maintained on a 12-hour light/dark cycle. Rabbits were maintained in accordance with the National Institutes of Health guidelines. All procedures were approved by the West Virginia University ACUC. All rabbits were initially given a standard Purina rabbit chow diet (contains $0 \%$ cholesterol) for the first ten days of training after which half were given a $2 \%$ cholesterol Purina rabbit chow diet (Dyets Inc.) and half were left on the standard Purina rabbit chow. All rabbits were maintained on their respective diet throughout the remainder of the experiment.

Apparatus: The apparatus and recording procedures for the rabbit NMR have been detailed by Schreurs and Alkon (1990) who modeled their apparatus after those described by Gormezano (Coleman \& Gormezano, 1971; Gormezano, 1966). Each subject was restrained in a Plexiglas box and trained in a sound-attenuating, ventilated chamber (Coulbourn Instruments, Model E10-20). A stimulus panel containing a speaker and houselights (10-W, 120-V incandescent lamps) was mounted at a 45 degree angle, $15 \mathrm{~cm}$ anterior to and $15 \mathrm{~cm}$ above the subject's head. An ambient noise level of $65 \mathrm{~dB}$ in each chamber was provided by an exhaust fan. Corneal air puff controlled by a programmable air pressure delivery system (Tescom Corp., Model ER-3000) was delivered through a tube ( $1 \mathrm{~mm}$ internal diameter) positioned $5 \mathrm{~mm}$ from and perpendicular to the center of 
the cornea. Air pressure intensity was measured at the orifice of the delivery tube in a closed system by a Smart Manometer (Meriam Instrument, 350 Series). Individual NM A/D outputs were stored on a trial-by-trial basis for subsequent analysis. Details of transducing nictitating membrane movements have been reported previously (Gormezano \& Gibbs, 1988; Schreurs \& Alkon, 1990). A hook connected to an L-shaped lever containing a freely moving ball and socket joint was attached to a 6-0 nylon loop sutured into, but not through, the nictitating membrane (NM). The other end of the lever was attached to a potentiometer (Novotechnik US Inc., Southborough, MA; Model P2201) that, in turn, was connected to a 12-bit analog-to-digital converter (5-ms sampling rate; 0.05-mm resolution). Data collection, analysis and stimulus delivery have been described (Buck et al., 2001) and were accomplished using a LabVIEW system (LabVIEW 5.1, National Instruments).

NMR classical conditioning: Rabbits received a day of adaptation, ten daily sessions of paired trace CS- US presentations $(n=12)$ or unpaired CS and US presentations $(n=12)$, and then after a two-month period of dietary supplementation, ten days of CS-alone extinction followed by brain tissue analysis. On adaptation day, the rabbits were prepared for AP and recording of NM movement and then adapted to chambers for the same time as subsequent training sessions (60 $\mathrm{min})$. Each of the paired trace conditioning sessions consists of 60 presentations of a $250-\mathrm{ms}, 1-\mathrm{KHz}, 82-\mathrm{dB}$, tone CS followed by a $250 \mathrm{~ms}$ trace interval and then a 100-ms, 4-psi air puff US. Sessions for unpaired subjects consisted of $60 \mathrm{CS}$-alone and 60 US-alone presentations that occurred in an explicitly unpaired manner delivered, on average, every 30 s. Rabbits were then reassigned so that 
terminal levels of responding within paired and unpaired groups were comparable before beginning the two month feeding period. One group of rabbits from each training condition received a $2.0 \%$ cholesterol diet and the other received a $0.0 \%$ cholesterol diet [control]. For all rabbits, CS extinction sessions consisted of 60, 250-ms, 1-KHz, 82-dB, tone alone presentations with a trial delivered, on average, every $60 \mathrm{~s}$. A conditioned response (CR) was defined as any extension of the NM exceeding $0.5 \mathrm{~mm}$ initiated after CS onset but before US onset for paired trials and the point at which the US would have occurred for CS-alone extinction trials. An unconditioned response (UR) was any extension of the NM exceeding $0.5 \mathrm{~mm}$ initiated after US onset.

Tissue collection: After the final day of CS-alone extinction, the animals were anesthetized with a solution containing Ketamine HCL $(83.33 \mathrm{mg} / \mathrm{ml})$ and Xylazine $(16.67 \mathrm{mg} / \mathrm{ml})$, followed by a lethal dose of Euthasol (sodium pentobarbital, $390 \mathrm{mg} / \mathrm{ml}$ ). Following transcardial perfusion with heparinized saline (10000units/2L), the brains were removed and the cerebellum, brainstem, hippocampi and a portion of rostral cortex were dissected. The tissue was weighed, immersed in $0.32 \mathrm{M}$ sucrose and frozen at $-20 \mathrm{C}$.

Subcellular fractionation: Myelin was purified from each brain region by the flotation technique (Konat, 1981). Briefly, the tissue was homogenized in $0.88 \mathrm{M}$ sucrose using 10 up-and-down strokes for 2.5 minutes. The homogenate was then transferred into centrifuge tubes by rinsing the homogenizer three times with $1 \mathrm{~mL}$ of $0.88 \mathrm{M}$ sucrose. The tubes were covered with parafilm, mixed well and kept on ice. The homogenate was 
overlaid with $0.32 \mathrm{M}$ sucrose (approximately $3 \mathrm{~mL}$ ) and centrifuged at 30,000 RPM for 1 hour at $4^{\circ} \mathrm{C}$ in a SW $40 \mathrm{Ti}$ Beckman rotor. The top $0.32 \mathrm{M}$ sucrose layer was removed with a syringe and discarded. The myelin interface was aspirated and transferred into new centrifuge tubes. The $0.88 \mathrm{M}$ supernatant sucrose layer was removed with a syringe and discarded. The non-myelin fraction (pellet) was suspended in $5 \mathrm{ml}$ of water and transferred into a new tube. The myelin and non-myelin fraction were diluted with up to $12 \mathrm{~mL}$ of distilled water. Tubes were centrifuged at $30,000 \mathrm{RPM}$ for 1 hour at $4^{\circ} \mathrm{C}$ in a SW 40 Ti rotor. The supernatant was discarded and the pellets were suspended in $1 \mathrm{~mL}$ of water using a $1 \mathrm{~mL}$ syringe. The volume of each tube was adjusted to $2 \mathrm{~mL}$ by weighing (using empty tube as tare and adding water to $2 \mathrm{~g}$ ).

Protein determination: Protein content of subfractions was quantified using a bicinchoninic acid (BCA) kit from Pierce (Rockford, IL). Briefly, a standard curve using an albumin standard $(2000 \mathrm{ug} / \mathrm{mL})$ was prepared by running duplicate albumin standards ranging from 0-160ug of protein. To each of the standard tubes was added 0, 10, 20, 30, $40,50,60,70$, or $80 \mathrm{uL}$ of albumin standard, $20 \mathrm{uL}$ of $10 \%$ SDS and then distilled water to make a final volume of $100 \mathrm{uL}$. A working reagent for protein determination was prepared by mixing $50 \mathrm{~mL}$ of Protein Reagent A with $1 \mathrm{~mL}$ of Protein Reagent B. $2 \mathrm{~mL}$ of this working reagent was applied to each of the standard samples. The samples were mixed and incubated at room temperature for exactly 2 hours in the dark and then read using a spectrophotometer at $562 \mathrm{~nm}$ against a reagent blank. The appropriate sample size to stay within the boundaries of the albumin standard curve for most of the brain regions to be analyzed was determined from the pilot study. All experimental samples were prepared 
using appropriate sample sizes, treated and read as the standards. Adjusting for sample sizes in each of the brain structures the amount of protein was determined by multiplying 92.5 to $\mathrm{OD}{ }_{562}$ of the sample readings.

Lipid extraction: Total lipids were extracted from the subfractions with chloroformmethanol (Konat and Clausen, 1974). Briefly, samples were suspended with a 1000uL pipettor 10 times minimizing foaming. $1 \mathrm{~mL}$ of each sample was transferred into a $20 \mathrm{~mL}$ glass labeled tube and $9 \mathrm{~mL}$ of chloroform methanol (1:1) was added to each sample. Samples were transferred to $15 \mathrm{~mL}$ centrifuge tubes vortexed for ten seconds and then centrifuged at 5000 RPM for 5 minutes at 25 degrees C. The supernatant was transferred into $20 \mathrm{~mL}$ glass tubes and $1 \mathrm{~mL}$ of water and $1 \mathrm{~mL}$ of chloroform: methanol: water (3:48:47) was added. Each tube was then vortexed three times for 10 seconds. Samples were transferred back to centrifuge tubes and centrifuged again at 5000 RPM for 5miutes at 25 degrees $\mathrm{C}$. Then using a glass pipette and one sample at a time the upper and interface layers were removed and discarded. The bottom layer (chloroform) was placed in glass capped tubes.

Cholesterol quantification: Cholesterol was quantified by the acetic anhydride (Eng \& Noble, 1968). Briefly, from each sample a portion of lipid extract (determined appropriate amount for myelin and nonmyelin portions for each structure to stay within standard curve range) was placed into a $10 \mathrm{~mL}$ glass tube and $2 \mathrm{~mL}$ of chloroform and $2 \mathrm{~mL}$ of acetic anhydride: sulfuric acid (20:1) was added. The samples were incubated in 
the dark for 20 minutes and read using a spectrophotometer at $620 \mathrm{~nm}$ against a reagent blank. The amount of cholesterol was determined $\left[\mathrm{x}: \mathrm{mol} \mathrm{Chol}=2.3 \mathrm{E}_{620}\right]$. The lipid content in the fractions was normalized to the amount of protein and expressed as nmols of lipid per mg of protein.

Sulfatide quantification: Sulfatides were determined by the Azure A method (Kean, 1968). Briefly, a sample of lipid extract was transferred into a screw-cap glass culture tube and evaporated to dryness under nitrogen at 55 degrees $\mathrm{C}$. Then to each tube was added $4 \mathrm{~mL}$ chloroform: methanol (1:1), $4 \mathrm{~mL} 0.05 \mathrm{~N}$ sulfuric acid and $0.8 \mathrm{~mL}$ of dye solution (20mg Azure A, $2.5 \mathrm{~mL}$ of $0.05 \mathrm{~N}$ sulfuric acid and distilled water to $50 \mathrm{~mL}$ ). Tubes were capped and vortexed for 1 minute. All samples were centrifuged at room temperature for 5 minutes using a table centrifuge. The upper phase was removed and discarded. Optical density was read at $645 \mathrm{~nm}$ against a reagent blank. The amount of sulfatides was determined $\left[\mathrm{x}: \mathrm{mol}\right.$ Sulf $\left.=27.8 \mathrm{E}_{645}\right]$. The lipid content in the fractions was normalized to the amount of protein and expressed as nmols of lipid per mg of protein.

Statistical analysis: Preliminary analysis indicated non-homogeneity of variance for both sulfatide and cholesterol levels. As a result, analyses were conducted on natural log transformed data. Results in this paper are stated in the original units of measurement. Analysis of variance (ANOVA) was performed and all post hoc analysis was done using statistical tests of contrasts with a Bonferroni correction between group means. 


\section{Results}

Behavior. Cholesterol retards recall of previously acquired classical conditioning of the rabbit NMR. Figure 5.1 shows acquisition (Panel A) and the effects of feeding a 2\%cholesterol diet for eight weeks on CS-alone extinction (Panel B) of the rabbit NMR. An ANOVA on the acquisition data yielded a significant main effect of pairings, $F(1,22)=$ $13.10, p<.005$, a significant interaction of days, $F(9,198)=31.12, p<.001$, and an interaction of pairings and days, $F(9,198)=19.08, p<.001$, confirming the clear differences in the levels of responding between the paired and unpaired rabbits. Panel B shows normal chow rabbits continued to respond at approximately $70 \%$ CRs over the first three days of extinction before decreasing over the remaining seven days. In contrast, cholesterol-fed rabbits responded at only $30 \%$ CRs on the first day of extinction, increased slightly to match normal chow levels of responding by the fourth day of extinction before declining with continued CS presentations. Responding to the tone in the unpaired groups was low and variable but there were no differences between groups. An ANOVA of the extinction data yielded a significant main effect of pairings, $F(1,20)$ $=21.30, p<.001$, a significant interaction of pairings with days, $F(9,180)=2.84, p<$ .01 , and an interaction of pairings with days and cholesterol, $F(9,180)=2.67, p<.01$, confirming the clear differences in the levels of responding between the cholesterol-fed paired rabbits and controls. Post hoc comparisons confirmed a significant difference between paired normal and paired cholesterol groups on the first and second days of extinction $(p$ 's $<.05)$.

The behavioral data suggest cholesterol has an effect on a previously acquired 
memory. The nature of this effect is open to interpretation because deficits in responding appear to be overcome, at least temporarily, with continued CS-alone presentations. It is possible that cholesterol induced a state-dependent effect as a result of weight loss, liver dysfunction or heart disease (Finking \& Hanke, 1997; Sparks, 1997b). However, the recovery of responding as a function of repeated CS-alone presentations is similar to normal rabbits that show recovery of CRs as a function of repeated CS-alone presentations after much longer retention intervals - as long as six months after trace conditioning (Schreurs, 1998) and even nine months after delay conditioning (Schreurs, 1993) . One might speculate that dietary cholesterol slowed retrieval of the memory so that after a two-month period the cholesterol fed rabbits had the retrieval issues of rabbits on a normal diet after six months. Importantly, we have shown that cholesterol-fed rabbits given CS-alone extinction immediately after tone-shock trace conditioning extinguish their CRs at the same rate and to the same level as normal diet controls (Schreurs et al., 2003).

Biochemical analysis. Although a comparison of brain sulfatide and cholesterol levels between the two diets did not reveal any significant differences (Figure 5.2), an ANOVA did reveal a significant difference in myelin, $F(1,20)=6.46, p<0.05$, and highly significant difference in non myelin brain cholesterol levels as a main effect of pairings, $F(1,20)=21.55, p<0.001$, and as an interaction of structure and pairings, $F(3,60)=$ $6.86, p<0.001$ and $F(3,60)=3.82, p<0.05$, respectively. Figure 5.3 shows cholesterol levels for paired and unpaired groups in the frontal lobe, hippocampus, brainstem, and cerebellum in myelin (panel A) and non myelin (panel B) fractions. Post hoc analysis of 
the interactions of structure and pairings confirmed brain cholesterol levels between the paired $(853.9 \pm 74.0 \mathrm{nmol} / \mathrm{mg})$ and unpaired groups $(575.9 \pm 28.3 \mathrm{nmol} / \mathrm{mg})$ in the hippocampus myelin fraction were significantly different, $p<0.05$. The analysis also confirmed a significant difference between the paired $(154.6 \pm 11.4 \mathrm{nmol} / \mathrm{mg})$ and unpaired groups $(98.9 \pm 6.2 \mathrm{nmol} / \mathrm{mg})$ in the frontal lobe non myelin fraction, $p<0.05$. A similar pattern of differences was found in the sulfatide levels as a function of pairing as shown in Figure 5.4. Statistical analysis revealed a significant difference in non myelin brain sulfatide levels as a main effect of pairing, $F(1,20)=28.64, p<0.001$, and an interaction of structure and pairing, $F(3,60)=3.28, p<0.05$. Post hoc analysis confirmed the difference between the paired $(7.3 \pm 0.52 \mathrm{nmol} / \mathrm{mg})$ and unpaired group $(4.8 \pm 0.32 \mathrm{nmol} / \mathrm{mg})$ in the frontal lobe was significant, $p<0.05$. Statistical analysis failed to find a difference in myelin brain sulfatide levels as a main effect of pairing, $F(1$, $20)=2.32, p=0.1434$, but there was as a significant interaction of structure and pairing, $F(3,60)=10.18, p<0.001$. Post hoc analysis affirmed, as suggested in Figure 4.4, that there was a significant difference between the paired $(100.9 \pm 7.5 \mathrm{nmol} / \mathrm{mg})$ and unpaired groups $(68.8 \pm 4.1 \mathrm{nmol} / \mathrm{mg})$ in the hippocampus, $p<0.05$. 


\section{Discussion}

Our study revealed two major findings. First, we show that dietary cholesterol negatively affects recall of previously acquired classical conditioning of the rabbit NMR. This is the first time the effects of dietary cholesterol have been assessed on previously formed memories. Prior to this work, all our observations about the effects of cholesterol on learning had been assessed on response acquisition i.e., the learning of a new memory (Schreurs et al., 2003; Schreurs et al., 2007b). Second, we show associative learningdependent changes in cholesterol and sulfatide levels in the hippocampus and frontal lobe. These are some of the first reported changes in cholesterol levels and, to our knowledge, the first reports of changes in sulfatide levels. It was interesting that we saw significant changes in both cholesterol and sulfatides specifically in the hippocampus and the frontal lobe since each is essential for some aspect of learning and memory, particularly the acquisition and extinction of trace conditioning (Bangasser et al., 2006; Cammarota et al., 2005; Frankland \& Bontempi, 2005; Izquierdo et al., 2007; Runyan et al., 2004; Thompson \& Kim, 1996).

Using our technique of separately analyzing myelin and myelin-free fractions, we did not find an effect of dietary cholesterol on brain cholesterol or sulfatide levels. There is research suggesting that dietary cholesterol affects brain cholesterol levels (Dufour et al., 2006; Sparks, 1997b); however, other work has failed to support this finding (Ghribi et al., 2006b). It is unclear at present how increases in dietary cholesterol and the subsequent increase in plasma lipid levels are signaled to the CNS to bring about cholesterol-mediated changes in the brain (Dietschy \& Turley, 2001). However, several 
hypotheses have been proposed. First, a number of researchers have demonstrated that although the BBB is usually impermeable to cholesterol, it's integrity is compromised in the cholesterol-fed rabbit (Ghribi et al., 2006a; Sparks et al., 2000a). Second, it has recently been demonstrated that there is a net flux of a cholesterol metabolite, 27hydroxycholesterol, from the circulation into the brain in healthy human volunteers (Heverin et al., 2005). More importantly, it has been demonstrated that mice fed a diet rich in cholesterol increased this natural flux of 27-hydroxycholesterol into the brain (Heverin et al., 2005). Third, it has been demonstrated that dietary manipulations can alter cell membrane composition in the brain (Faulks et al., 2006). Therefore, it is possible that alterations in cholesterol homeostasis in the CNS may affect the composition of neuronal membranes by increasing or decreasing cholesterol and other lipids to maintain rigidity and function (Hayashi et al., 2002) including synaptic plasticity (Frank et al., 2008).

Interestingly, our data indicate clear evidence of associative learning-dependent changes in both cholesterol and sulfatide levels. Since brain cholesterol levels are increased in the paired animals that learn the hippocampally-dependent task, it is possible that these increases are the result of synaptic plasticity and axonal growth (Frank et al., 2008; Koudinov \& Koudinova, 2001). Recently it was demonstrated that enzymes essential to cholesterol synthesis such as HMG-CoAR are present in adult neurons and are most prominently localized in cortical and hippocampal neurons (Korade et al., 2007). Cholesterol is essential for synaptic plasticity (Frank et al., 2008; Koudinov \& Koudinova, 2001; Koudinov \& Koudinova, 2003; Koudinov \& Koudinova, 2005) and Dufour and colleagues (2006) have illustrated a correlation between an increase in 
hippocampal brain cholesterol levels and memory changes using rats fed a regular diet. Therefore it is possible that hippocampal neurons, as well as cortical neurons, utilize more cholesterol in response to associative learning (Inda et al., 2005) even if most neuronal cholesterol is shuttled to the neuron from glial cells (Pfrieger, 2002).

Given that cholesterol and sulfatide analysis only took place at the conclusion of CS extinction, it is not possible to parse out the relative contribution of acquisition and extinction to the cholesterol and sulfatide differences. Nevertheless, it is interesting to speculate about the relative contribution of these processes to the myelinated and nonmyelinated fraction results. Increases in brain cholesterol and sulfatide levels in the hippocampal myelin fractions could be the result of trace conditioning, whereas the increases in the frontal lobe non myelin fraction could be the results of extinction. As discussed in the Introduction, both cholesterol and sulfatides have important roles in learning and memory. Brain maturation is not achieved until 13 to 26 weeks after birth in most mammals and not for many years in humans, during which time there is an excess amount of cholesterol in the CNS (Dietschy, 2009). Furthermore, studies have shown that increases in the levels of white matter are correlated with acquiring new skills (Als et al., 2004; Bengtsson et al., 2005). It is therefore possible that training young rabbits allows for incorporation of cholesterol and sulfatides into cellular membranes and myelin during learning.

The role of the hippocampus in learning a difficult behavioral task (such as trace conditioning) has been well documented (Bangasser et al., 2006; Frankland \& Bontempi, 2005; Runyan et al., 2004). Using rabbits, one study illustrated how large bilateral lesions of the hippocampus made before training seriously impaired learning of a trace 
conditioning task. When the rabbits were trained first, lesions made immediately after training abolished the ability to remember the difficult task; however, lesions made one month after training had no effect on memory retention of the previously learned difficult task (Kim et al., 1995). The role of the frontal lobe, specifically the prefrontal lobe, in learning and memory has been well documented (Bangasser et al., 2006; Cammarota et al., 2005; Frankland \& Bontempi, 2005; Izquierdo et al., 2007; Runyan et al., 2004; Thompson \& Kim, 1996). The forebrain also plays a role in trace conditioning and extinction (Gruart et al., 2000; Weible et al., 2000). Given that myelin formation and change takes considerable time and trace conditioning occurred more than two months before tissue analysis, one could argue that increases in hippocampal brain cholesterol and sulfatide levels would be seen in the myelin fraction. On the other hand, since extinction occurred immediately before tissue analysis, increases in cholesterol and sulfatide levels may only have been seen in the non myelin fraction.

Taken together, our results show that dietary cholesterol appears to have a positive effect on learning but a negative effect on memory recall. How is this possible? As already noted, there are a large number of studies that have documented significant CNS as well as systemic consequences of a high-cholesterol diet. The CNS effects include, but are not limited to, compromise of the BBB (Ghribi et al., 2006a; Sparks et al., 2000a), increases in cholesterol metabolites such as 27-hydroxycholesterol (Heverin et al., 2005), an elevation in inflammatory markers (Rahman et al., 2005; Yehuda et al., 2005), the accumulation of A $\beta$ (Schreurs et al., 2007a; Sparks \& Schreurs, 2003), damage to the myelin sheath and axon (Berkman et al., 2009), and changes in membrane cholesterol (Ghribi et al., 2006b). Some of these processes take place within days of 
starting the diet whereas others may take weeks. It is therefore possible that when animals are fed cholesterol immediately after training, acquisition-specific changes in the hippocampus or long-term storage changes in the prefrontal cortex may be affected by the rapidly increasing CNS consequences of the diet. So when assessed for memory recall, animals perform poorly. On the other hand, when animals are placed on a cholesterol diet for two months before training, learning-specific changes are more likely to occur against a backdrop of steady-state cholesterol-induced CNS changes. We have shown that the training-specific changes remain intact and even benefit from the consequences of the diet when cholesterol-fed rabbits show better acquisition than controls (Schreurs et al., 2007a). These effects could be the result of the increased membrane cholesterol that is important for synaptic plasticity. If the cholesterol diet induces $A \beta$ plaques, we have found that rabbits show significantly worse acquisition than controls (Schreurs et al., 2007a; Sparks \& Schreurs, 2003). 


\section{Figure 5.1}

\section{A}

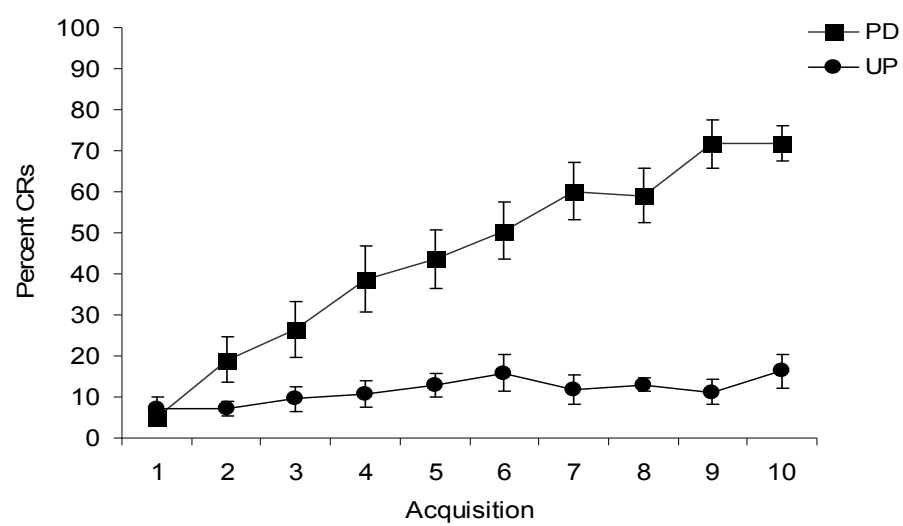

B

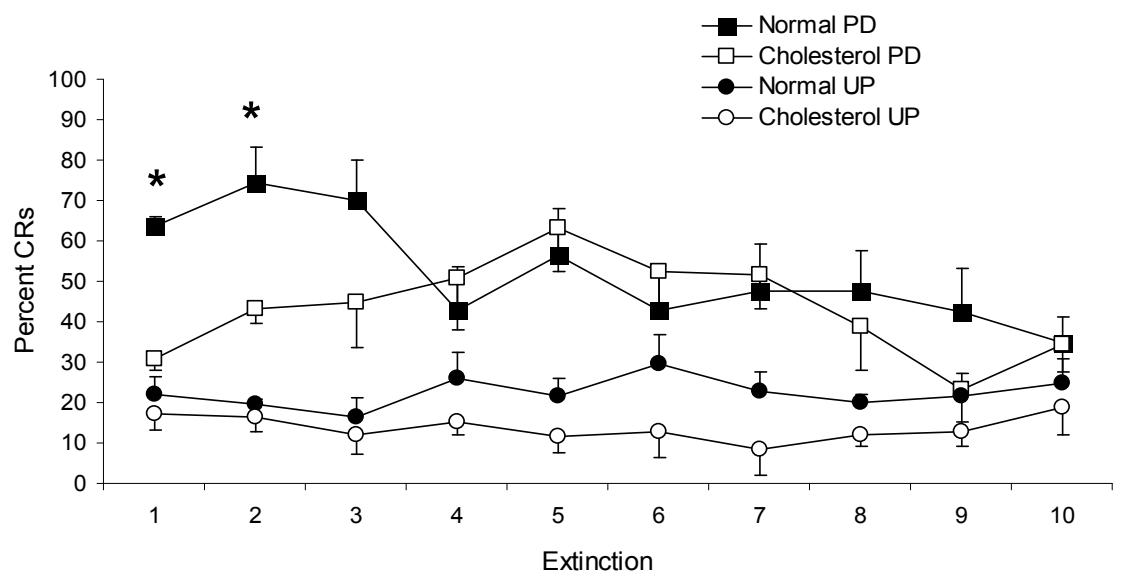

Figure 5.1. Mean conditioned responses in acquisition and extinction. Acquisition phase (A) of the twelve rabbits that received 60 daily pairings of a $250-\mathrm{ms}, 82-\mathrm{dB}$, tone CS and 100-ms, 4-psi air puff US separated by a 250 -ms trace (500-ms ISI, PD). Another twelve rabbits received 120 daily explicitly unpaired presentations of the tone and air puff (UP). Half the rabbits in each group were then placed on a $2 \%$ cholesterol diet (Cholesterol) and the other half remained on their normal diet ( $0 \%$ cholesterol, Normal). Extinction phase (B) of the twenty-four rabbits that received pairings $(\mathrm{n}=12)$ or unpaired presentations $(\mathrm{n}=12)$. Following an 8 -week $2 \%$ cholesterol diet, rabbits previously conditioned to a tone CS show significantly lower levels of responding to the tone CS during tone-alone extinction. ${ }^{*} p<.05$ post hoc comparison of percent CRs between paired groups on day 1 and day 2 of extinction. 


\section{Figure 5.2}

A

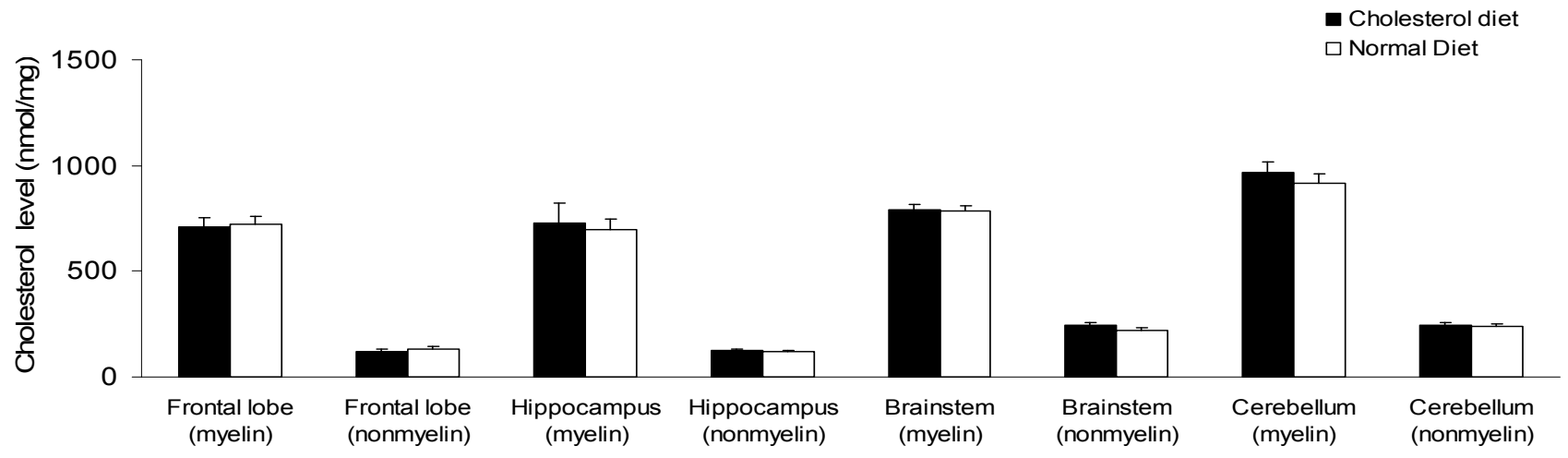

B

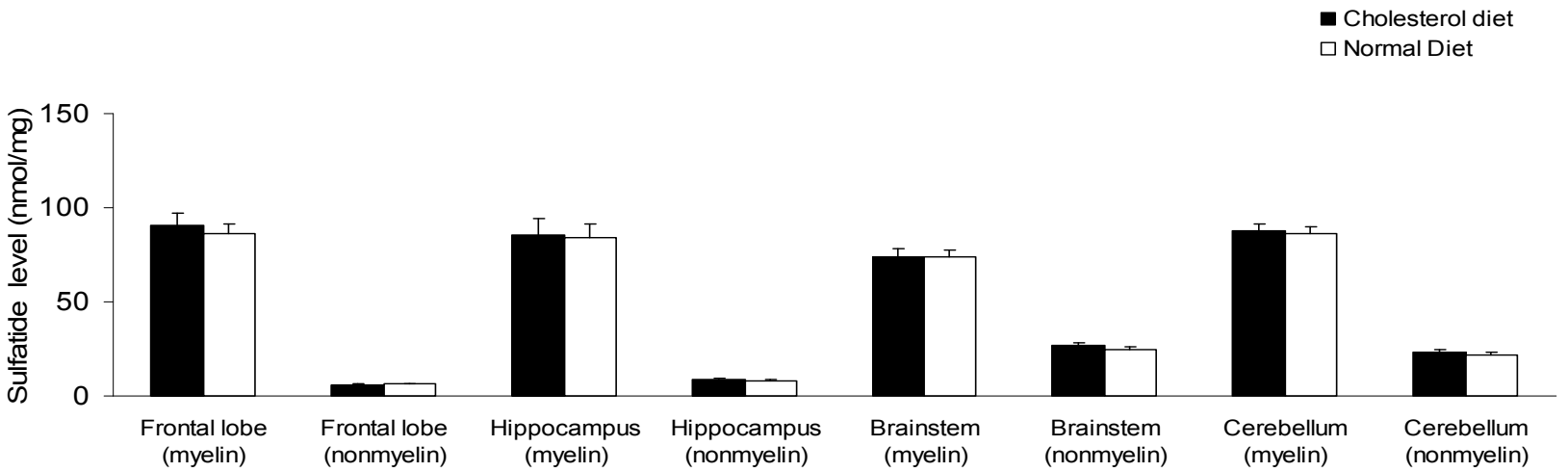

Figure 5.2. Cholesterol and sulfatides levels as a function of diet. Cholesterol and sulfatides were extracted separately from myelin and non myelin fractions of the frontal lobe, hippocampus, brainstem, and cerebellum. These lipids were quantified as described in the Methods section and expressed as nmoles per mg of protein. Analysis of cholesterol levels in myelin and non myelin fractions $(\mathrm{A})$ as a function of diet revealed no significant differences in any of the fractions (n's = 12). Similarly, no differences in sulfatide levels (B) as a function of diet were found in any of the myelin and non myelin fractions among the different structures (n's = 12). 


\section{Figure 5.3}

A

$\square$ PD $\quad$ UP

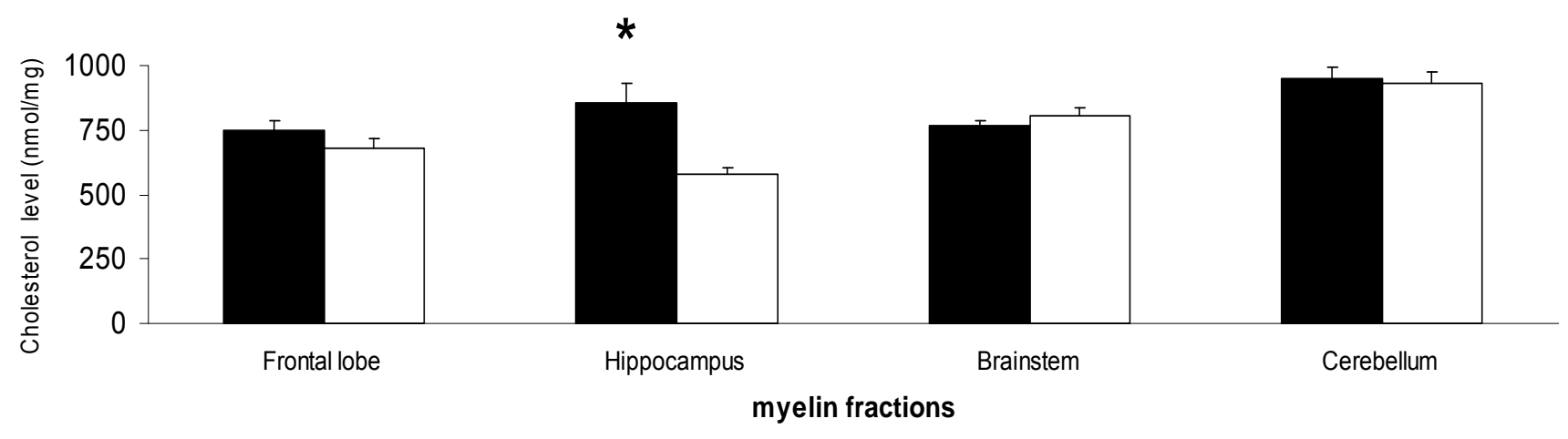

B

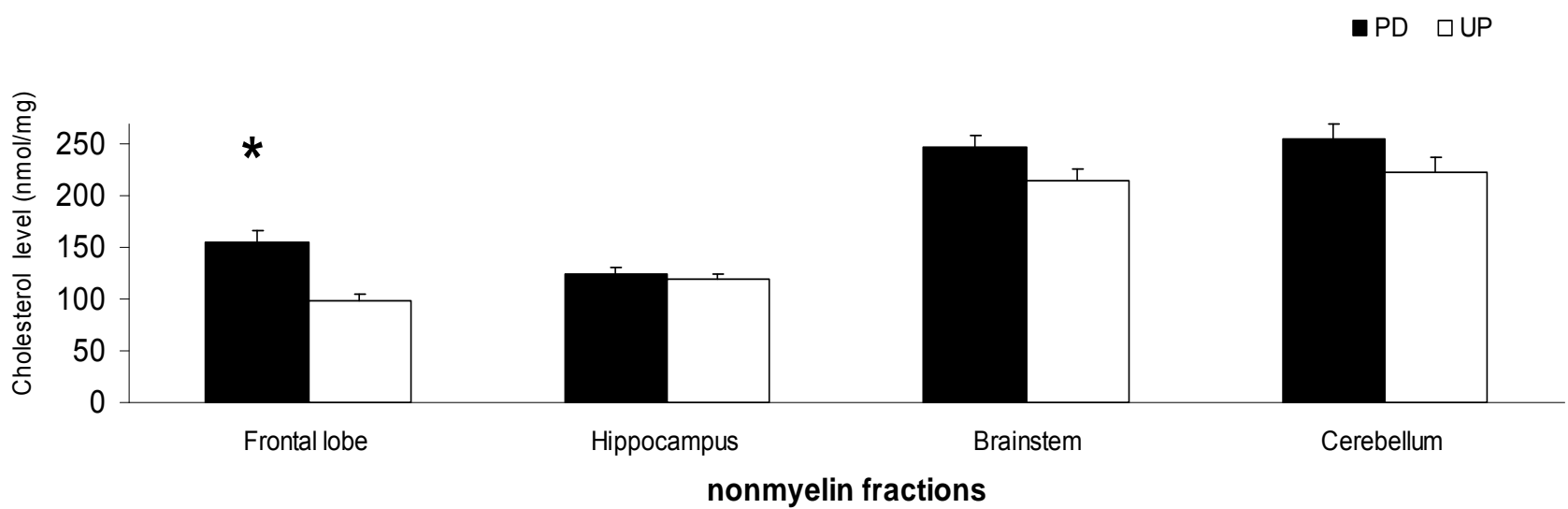

Figure 5.3. Cholesterol levels as a function of pairing. Cholesterol was extracted separately from myelin and non myelin fractions of the frontal lobe, hippocampus, brainstem, and cerebellum and quantified as described in the Methods section and expressed as nmoles per mg of protein. Analysis of cholesterol levels in the myelin fractions (A) as a function of pairing revealed a significant difference in the hippocampus between paired and unpaired groups (n's $=12$ ). Analysis of cholesterol levels in the non myelin fractions (B) as a function of pairing revealed a significant difference in the frontal lobe (n's $=12) .{ }^{*} p<.05$. 


\section{Figure 5.4}

A

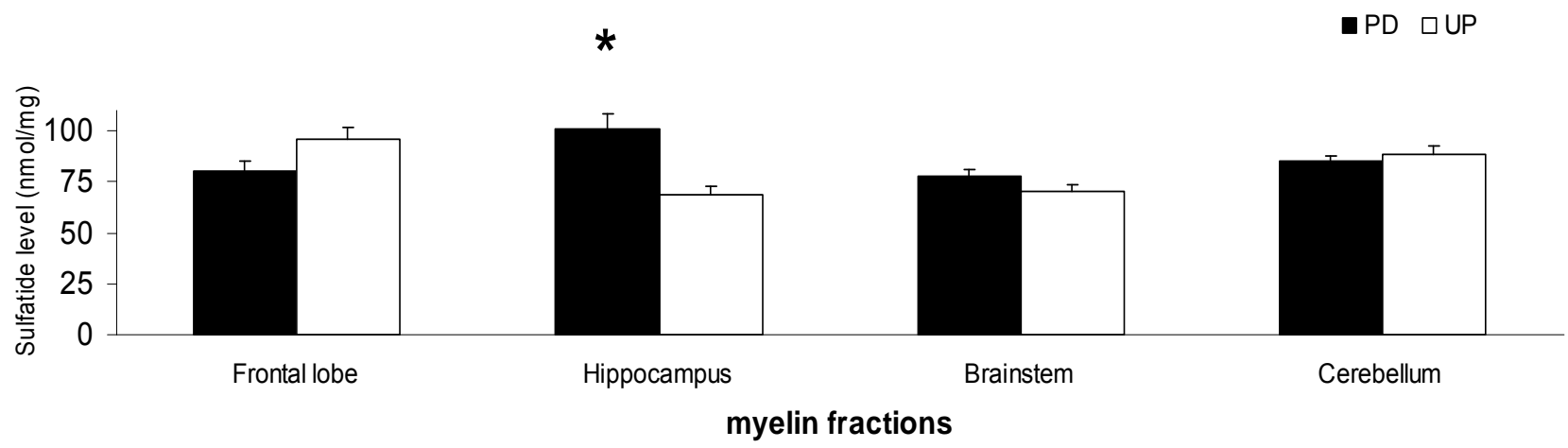

B

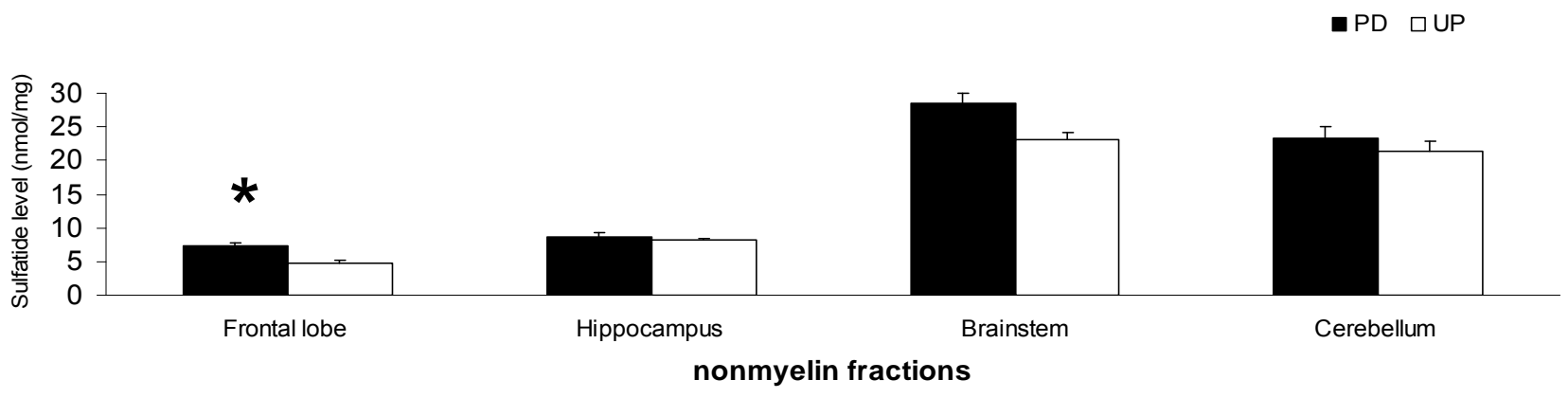

Figure 5.4. Sulfatide levels as a function of pairing. Sulfatides were extracted separately from myelin and non myelin fractions of the frontal lobe, hippocampus, brainstem, and cerebellum and quantified as described in the Methods section and expressed as nmoles per mg of protein. Analysis of sulfatide levels in the myelin fractions (A) as a function of pairing revealed a significant difference in the hippocampus between paired and unpaired groups ( $\mathrm{n}$ 's $=12$ ). Analysis of sulfatide levels in the non myelin fractions (B) as a function of pairing revealed a significant difference in the frontal lobe (n's $=12) .{ }^{*} p<.05$. 


\section{Chapter Six}

Trace Conditioning Alone Does Not Increase Brain Sulfatide and Cholesterol Levels

This work is in preparation. 


\begin{abstract}
The results from our last study presented in Chapter 5 suggested an increase in sulfatide and cholesterol levels as a direct function of associative learning and memory recall. In those experiments, the animals were exposed to several training paradigms (both trace conditioning and extinction) and all perfused after assessing memory recall. Given that these training paradigms are both hippocampal- and frontal lobe-dependent, we investigated whether the learning associated variations in brain cholesterol and sulfatide levels were a direct function of trace conditioning alone, absent the memory recall task, in four major brain structures (hippocampus, frontal lobe, brainstem, and cerebellum). We found that trace conditioning alone did not produce any detectable changes in sulfatide or cholesterol levels as a function of learning. We also investigated whether these changes in brain chemistry occurred a day after training or required the 9 weeks and two days seen in the previous experiment. We found some significant changes in the sulfatide levels as a function of time, but not in the cholesterol levels. The data suggest that the changes we saw in sulfatide and cholesterol levels in the previous experiment may require the combination of trace conditioning and extinction and that sulfatide level change may be time-dependent.
\end{abstract}




\section{Introduction}

In the past several decades, brain cholesterol and sulfatides have been shown to have a role in learning and memory. Furthermore, in recent years there is growing evidence of a strong link between cholesterol and sulfatides and neurological diseases, such as AD. Disturbances in brain cholesterol are thought to lead to increases in $A \beta$ - a protein linked to AD. Similarly, disturbances in brain sulfatide levels have numerous effects on learning and memory and have been linked to AD. To better understand the role of cholesterol and sulfatides in neurological diseases, we need to better understand their role in learning and memory.

Cholesterol and sulfatide levels in the CNS are tightly controlled. Cholesterol is a large, hydrophobic, steroid molecule that is important for cell structure, repair and signaling, myelin synthesis, synaptic plasticity, and hormone and neurosteriod production. Disturbances in cholesterol levels are correlated with disorders in humans such as Niemann-Pick disease Type C, Smith-Lemli-Opitz syndrome and AD (Ikonen, 2006; Mori et al., 2001; Papassotiropoulos et al., 2002; Voikar et al., 2002; Whitney et al., 2002; Xie et al., 2000). Sulfatides are a class of sulfated galactocerebrosides that have an important role in myelin sheath and axon structure maintenance, modulation of potassium and sodium ion channels, and have been shown to be altered in a number of neurological disorders including MLD, PD, and AD.

Individual brain structures play specific roles in rabbit NM classical conditioning. The neural substrates of rabbit NM classical conditioning include the brainstem, cerebellum and hippocampus (Berger et al., 1991; Berger \& Orr, 1983; Cavallaro et al., 
2001). The forebrain also plays a role in rabbit NM classical conditioning (Gruart et al., 2000). The involvement of the brainstem and cerebellum is necessary for rabbit NM classical conditioning: however, involvement of the hippocampus in NM classical conditioning is dependent upon the complexity of the task (Beylin et al., 2001). For example, although the hippocampus may not be necessary for the acquisition of a $\mathrm{CR}$ during NM delay conditioning (where the CS and the US overlap), it is necessary for CR acquisition during NM trace conditioning (where there is a temporal gap between the CS and US) (Bangasser et al., 2006). The forebrain also plays a key role in NM trace conditioning and extinction (where CS-alone trials are presented after conditioning) (Gruart et al., 2000).

We do not yet know if brain cholesterol and sulfatide levels change from trace conditioning of the NM alone in learning and memory related brain structures. The results from our study presented in the previous chapter suggest an increase in sulfatide and cholesterol levels as a function of trace conditioning in combination with extinction. In those experiments, the animals were exposed to both trace conditioning and extinction and then perfused immediately. Given that these training paradigms are both hippocampal and frontal lobe dependent, in the following study we investigated whether the learning associated variations in brain cholesterol and sulfatide levels were a direct function of trace conditioning alone in four major brain structures (hippocampus, frontal lobe, brainstem, and cerebellum). We found that trace conditioning alone did not produce any changes in sulfatide or cholesterol levels as a function of learning We also investigated whether changes in brain chemistry occurred a day after training or required the 9 weeks and two days seen in the previous experiment. We found some significant 
changes in the sulfatide levels as a function of time, but not in the cholesterol levels. The data suggest that the changes we saw in sulfatide and cholesterol levels the previous experiment may require the combination of trace conditioning and extinction and that sulfatide level changes maybe time sensitive. 


\section{Methods}

Subjects: Subjects were 24 male New Zealand white rabbits (Oryctolagus cuniculus) weighing approximately $2.0-2.5 \mathrm{~kg}$ at the beginning of the experiment. Animals were housed in individual cages, given free access to food and distilled water, and maintained on a 12-hour light/dark cycle. Rabbits were maintained in accordance with the National Institutes of Health guidelines. All procedures were approved by the West Virginia University ACUC. All animals were placed on a standard Purina rabbit chow (contains $0 \%$ cholesterol) and maintained on this diet throughout the course of the experiment.

Apparatus: The apparatus and recording procedures for the rabbit NMR have been detailed by Schreurs and Alkon (1990) who modeled their apparatus after those described by Gormezano (Coleman \& Gormezano, 1971; Gormezano, 1966). Each subject was restrained in a Plexiglas box and trained in a sound-attenuating, ventilated chamber (Coulbourn Instruments, Model E10-20). A stimulus panel containing a speaker and houselights (10-W, 120-V incandescent lamps) was mounted at a 45 degree angle, $15 \mathrm{~cm}$ anterior to and $15 \mathrm{~cm}$ above the subject's head. An ambient noise level of $65 \mathrm{~dB}$ in each chamber was provided by an exhaust fan. Corneal air puff controlled by a programmable air pressure delivery system (Tescom Corp., Model ER-3000) was delivered through a tube ( $1 \mathrm{~mm}$ internal diameter) positioned $5 \mathrm{~mm}$ from and perpendicular to the center of the cornea. Air pressure intensity was measured at the orifice of the delivery tube in a closed system by a Smart Manometer (Meriam Instrument, 350 Series). Individual NM A/D outputs were stored on a trial-by-trial basis for subsequent analysis. Details of 
transducing nictitating membrane movements have been reported previously (Gormezano \& Gibbs, 1988; Schreurs \& Alkon, 1990). A hook connected to an L-shaped lever containing a freely moving ball and socket joint was attached to a 6-0 nylon loop sutured into, but not through, the nictitating membrane (NM). The other end of the lever was attached to a potentiometer (Novotechnik US Inc., Southborough, MA; Model P2201) that, in turn, was connected to a 12-bit analog-to-digital converter (5-ms sampling rate; 0.05-mm resolution). Data collection, analysis and stimulus delivery have been described (Buck et al., 2001) and were accomplished using a LabVIEW system (LabVIEW 5.1, National Instruments).

NMR classical conditioning: Rabbits received a day of adaptation, ten daily sessions of paired trace CS- US presentations $(n=12)$ or unpaired CS and US presentations $(n=12)$ after which half the rabbits were euthanized the day after and the other half of the rabbits were euthanized 9 weeks and two days later (to be equal in time of perfusion in the first experiment) the other half of the rabbits were euthanized. On adaptation day, the rabbits were prepared for AP and recording of NM movement and then adapted to chambers for the same time as subsequent training sessions $(60 \mathrm{~min})$. Each of the paired trace conditioning sessions consists of 60 presentations of a $250-\mathrm{ms}, 1-\mathrm{KHz}, 82-\mathrm{dB}$, tone CS followed by a $250 \mathrm{~ms}$ trace interval and then a 100-ms, 4-psi air puff US. Sessions for unpaired subjects consisted of $60 \mathrm{CS}$-alone and $60 \mathrm{US}$-alone presentations that occurred in an explicitly unpaired manner delivered, on average, every $30 \mathrm{~s}$. Afterwards rabbits were then assigned to groups so that the initial $\% \mathrm{CR}$ among groups was equal. Half the rabbits were perfused the day after and the other half was perfused 9 weeks and two days 
later. A conditioned response (CR) was defined as any extension of the NM exceeding $0.5 \mathrm{~mm}$ initiated after CS onset but before US onset.

Tissue collection: The animals were anesthetized with a solution containing Ketamine HCL $(83.33 \mathrm{mg} / \mathrm{ml})$ and Xylazine $(16.67 \mathrm{mg} / \mathrm{ml})$, followed by a lethal dose of Euthasol (sodium pentobarbital, $390 \mathrm{mg} / \mathrm{ml}$ ). Following transcardial perfusion with heparinized saline, the brains were removed and the cerebellum, brainstem, hippocampi and a portion of rostral cortex were dissected. The tissue was weighed, immersed in $0.32 \mathrm{M}$ sucrose and frozen at $-20 \mathrm{C}$.

Subcellular fractionation: Myelin was purified from each brain region by the flotation technique (Konat, 1981). Briefly, the tissue was homogenized in $0.88 \mathrm{M}$ sucrose, overlaid with $0.32 \mathrm{M}$ sucrose and centrifuged at $30,000 \mathrm{rpm}$ for 1 hour at $4{ }^{\circ} \mathrm{C}$ in a SW $40 \mathrm{Ti}$ Beckman rotor. The myelin fraction located at the gradient interface and the non myelin fraction (pellet) were collected and washed free of sucrose. Protein content was determined using a bicinchoninic acid (BCA) kit from Pierce (Rockford, IL).

Lipid analysis: Total lipids were extracted from the subfractions with chloroformmethanol, and cholesterol and sulfatides were quantified by the acetic anhydride and Azure A methods, respectively as previously described in Chapter 5. 
Statistical analysis: Preliminary analysis indicated non-homogeneity of variance for both sulfatide and cholesterol levels. As a result, analyses were conducted on natural log transformed data. Results in this paper are stated in the origin units of measurement. Analysis of variance (ANOVA) was performed and all post hoc analysis was done using statistical tests of contrasts with a Bonferroni correction between group means. 


\section{Results}

Behavior. Animals receive the same hippocampus dependent training (trace conditioning) as in Chapter 5 Experiment 1. Twenty four rabbits were assigned to two groups comprising pairings (Paired vs Unpaired) and perfusion time (A day after training vs two months later) in a 2 X 2 factorial design. As illustrated in Figure 6.1 responding for paired rabbits across 10 days of trace conditioning reached a mean level of $62 \%$ CRs. Training was terminated at this point to ensure the same duration of conditioning as in Experiment 1. Rabbit conditioning levels for the paired groups were counterbalanced between the two perfusion times to have an equal mean level of conditioning ( $62 \% \mathrm{CRs})$. Half the rabbit were then perfused immediately and the other half were kept for 9 weeks and two days to equate the post-training duration with our last experiment.

Biochemical analysis. ANOVAs with the factors of perfusion time $\mathrm{x}$ pairing $\mathrm{x}$ structure for the myelin and nonmyelin portions yielded no significant differences in brain cholesterol or sulfatide levels in any of the structures as a main effect of perfusion time or pairing (pairing data are illustrated in Figure 6.2). However as shown in Figure 6.3, the analysis of sulfatides (but not cholesterol) showed significant interaction of structure $\mathrm{x}$ time in both the myelin and nonmyelin portions, $F(3,60)=5.16, p<.01$, and , $F(3,60)=$ $5.41, p<.01$, respectively. Specifically, in the myelin portion (panel A), the difference was in the frontal lobe between those animals perfused the day after training $(76.6 \pm 5.3$ $\mathrm{nmol} / \mathrm{mg})$ and those perfused 9 weeks and 2 days later $(88.4 \pm 2.6 \mathrm{nmol} / \mathrm{mg}), \mathrm{p}<0.05$. In the nonmyelin portion (panel B), the differences were in the frontal lobe between those 
animals perfused the day after training $(7.7 \pm 0.4 \mathrm{nmol} / \mathrm{mg})$ and those perfused 9 weeks and 2 days later $(9.8 \pm 0.5 \mathrm{nmol} / \mathrm{mg})$ and in the cerebellum between those animals perfused the day after training $(11.3 \pm 0.4 \mathrm{nmol} / \mathrm{mg})$ and those perfused 9 weeks and 2 days later $(13.3 \pm 0.3 \mathrm{nmol} / \mathrm{mg}), \mathrm{p}<0.05$.

It is possible that we did not see significant effects as a function of pairing due to our smaller sample sizes. In our previous experiment our sample sizes each contained 12 subjects where as in this experiment our sample sizes that equate the post-training duration with our last experiment each contained 6 subjects. A simple t-test comparing means of myelin sulfatide levels in the hippocampus between paired and unpaired groups revealed that the two groups were significantly different from one another, $\mathrm{p}<0.05$. Furthermore, a retrospective power analysis on the cholesterol and sulfatide levels as a function of pairings in the animals that equate the post-training duration with our last experiment indicated a lack of power in the myelin cholesterol levels in the hippocampus. Sample sizes of 6 indicated a $41 \%$ chance of rejecting the null hypothesis while sample sizes of 12 indicated a $74 \%$ chance of rejecting the null hypothesis. The power analysis also indicated a lack of effect in the nonmyelin cholesterol and sulfatide levels in the frontal lobe. A sample size of up to 100 still only indicated a $34 \%$ chance of rejecting the null hypothesis. 


\section{Discussion}

The study revealed several interesting findings. First, the study showed that sulfatide levels in both the myelin and nonmyelin portions of the frontal lobe and the nonmyelin portion of the cerebellum all increase significantly as a function of time. Second, the study showed that learning and memory-dependent increases in cholesterol and sulfatide levels do not occur from trace conditioning alone. These findings in combination with our earlier findings suggest that changes in brain cholesterol and sulfatide levels require the combination of trace conditioning and extinction (memory recall task). However, the results also suggest that sulfatide levels change with time and so correlating sulfatide changes with learning and memory tasks that span a period of time maybe difficult.

The changes in sulfatide levels as a function of time appeared in both myelin and nonmyelin portions. Given that myelin formation and change takes considerable time, it is not surprising that more changes appeared in the non myelin portions, specifically in the frontal lobe and the cerebellum. It was also not surprising to see some changes in the myelin portions over time because cholesterol accounts for one third of myelin lipids and sulfatides and its precursor Gal C also account for almost one third of myelin lipids (Eckhardt, 2008). Sulfatides have an important role in myelin sheath and axon structure maintenance (Marcus et al., 2006) so it is reasonable to see some changes in their levels as a function of time.

Our results show that brain sulfatide levels but not cholesterol levels change with time. How is this possible? Both cholesterol and sulfatides have physiological functions in the CNS. However, sulfatides have a significant role in myelin maintenance (Marcus et 
al., 2006), whereas cholesterol is mainly a structural component of myelin and changes as a function of time may not be as apparent. Moreover, in this experiment we did not see changes of sulfatide or cholesterol levels as a function of learning. It may be that trace conditioning alone does not induce enough changes to be detected and the impact of presenting several tasks that require the overlap of the same learning related structure induce the changes we saw in our previous experiment. Other work has suggested that the combination of tone alone and trace conditioning in rats appears to induce an increase in hippocampal neurogenesis more than exposure to trace conditioning alone (Waddell \& Shors, 2008). The dentate gyrus of the hippocampus is thought to produces thousands of granule neurons each day. Many of these cells only survive a short period; however it has been shown that survival is enhanced by hippocampal-dependent trace classical conditioning (Gould et al., 1999; Leuner et al., 2004). Waddell and Shors (2008) conducted an experiment illustrating trace conditioning contributed to increased survival of cells in the dentate gyrus. Furthermore, their work also suggested the duration of hippocampal activation plays a factor in enhancing survival of newly generated cells in the dentate gyrus. Theoretically, combining a trace conditioning task with extinction increases the duration of hippocampal activation. This translates into an increase in survival of neurons which hypothetically would lead to increases in hippocampal brain cholesterol and sulfatide levels since both are major components of cellular membranes. Other mechanisms of synaptic plasticity, such as long-term potentiation, have also been shown to be dependent on cholesterol levels. Several studies have reported that depletion of cholesterol inhibits long-term potentiation and other forms of synaptic plasticity (Frank et al., 2008; Koudinov \& Koudinova, 2005). Therefore quantification of brain 
lipids such as cholesterol could provide further evidence of synaptic plasticity and neurogenesis.

As stated in the results section, it is also possible that we did not see significant effects as a function of pairing due to our smaller sample sizes. In our previous experiment our sample sizes each contained 12 subjects where as in this experiment our sample sizes that equate the post-training duration with our last experiment each contained 6 subjects. A simple t-test comparing means of myelin sulfatide levels in the hippocampus between paired and unpaired groups revealed that the two groups were significantly different from one another, $p<0.05$. Furthermore, a retrospective power analysis on the cholesterol and sulfatide levels as a function of pairings in the animals that equate the post-training duration with our last experiment indicated a lack of power in the myelin cholesterol levels in the hippocampus. The power analysis also indicated a lack of effect in the nonmyelin cholesterol and sulfatide levels in the frontal lobe. It is therefore necessary to replicate our findings before making any final conclusions. 
Figure 6.1

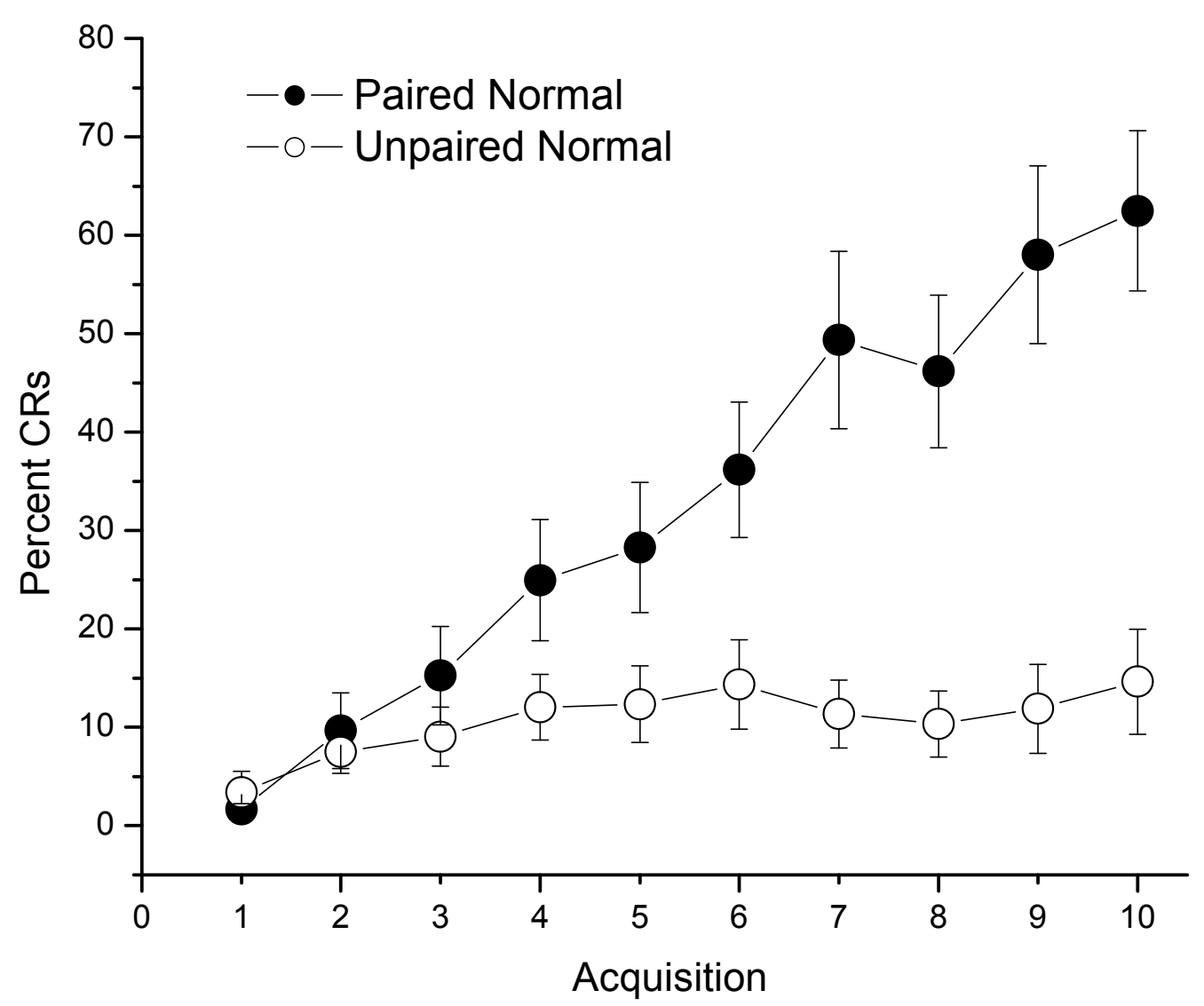

Figure 6.1. Mean percent conditioned responses from trace conditioning. Rabbits received the same trace conditioning as in Experiment 1.Twelve rabbits received 60 daily pairings of a 250-ms, 82-dB, tone CS and 100-ms, 4-psi air puff US separated by a 250ms trace (500-ms ISI) for ten days. Another twelve rabbits received 120 daily explicitly unpaired presentations of the tone and air puff. Rabbits were then reassigned to groups so that terminal levels of responding were comparable across groups. One group of paired and one group of unpaired rabbits were perfused the day after training and the other two groups were perfused 9 weeks and two days after training making time of perfusion equivalent to the groups in first experiment. 


\section{Figure 6.2}

A

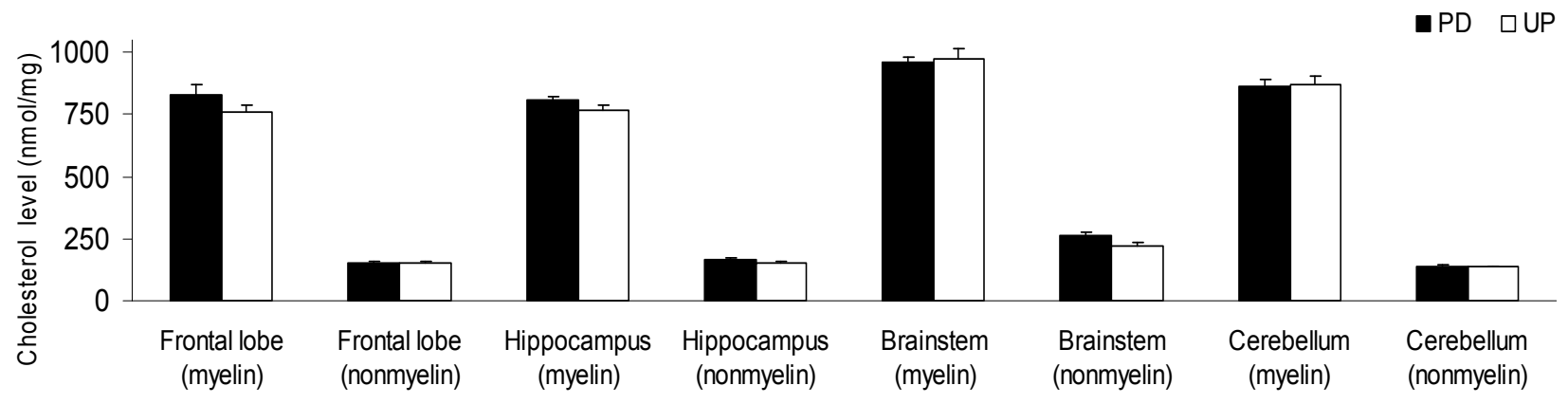

B

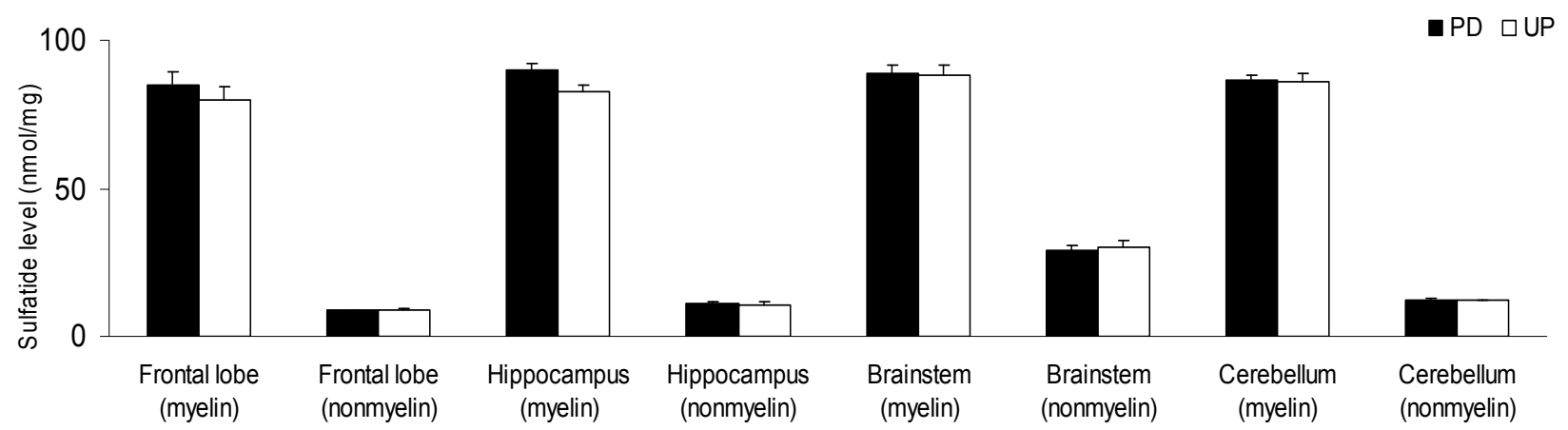

Figure 6.2. Cholesterol and sulfatides levels as a function of pairing. Cholesterol and sulfatides were extracted separately from myelin and non myelin fractions of the frontal lobe, hippocampus, brainstem, and cerebellum. These lipids were quantified as described in the Methods section and expressed as nmoles per mg of protein. Analysis of cholesterol levels in myelin and non myelin fractions (A) as a function of pairing revealed no significant differences in any of the fractions (n's = 12). Similarly, no differences in sulfatide levels $(B)$ as a function of pairing were found in any of the myelin and non myelin fractions among the different structures (n's $=12)$. 


\section{Figure 6.3}

A

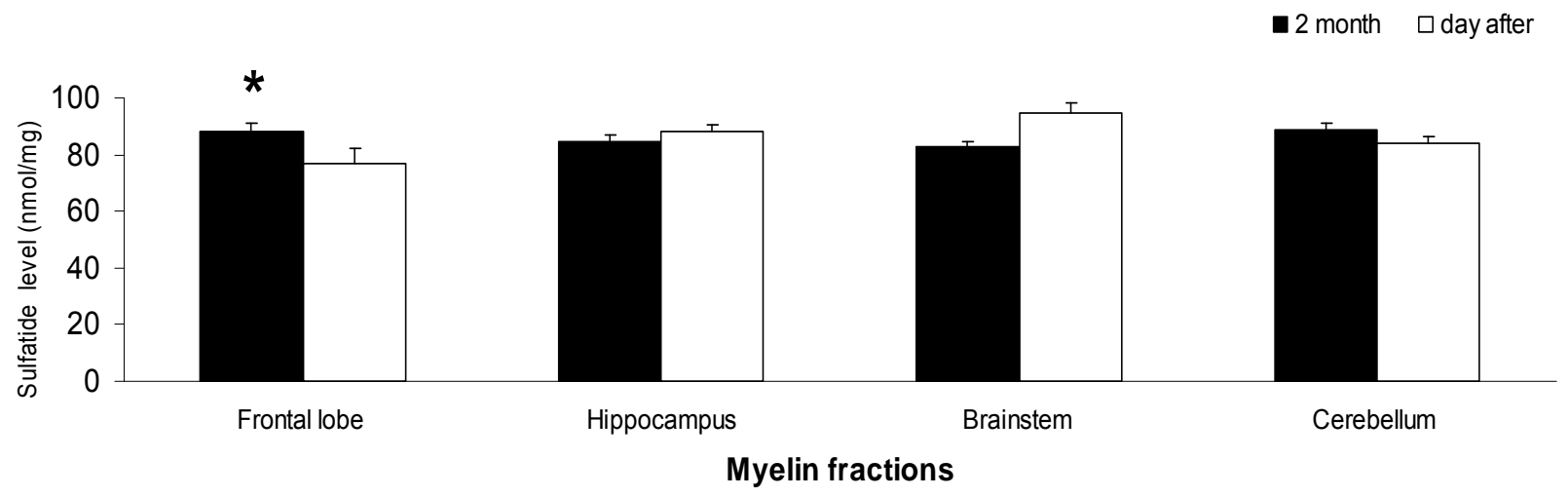

B

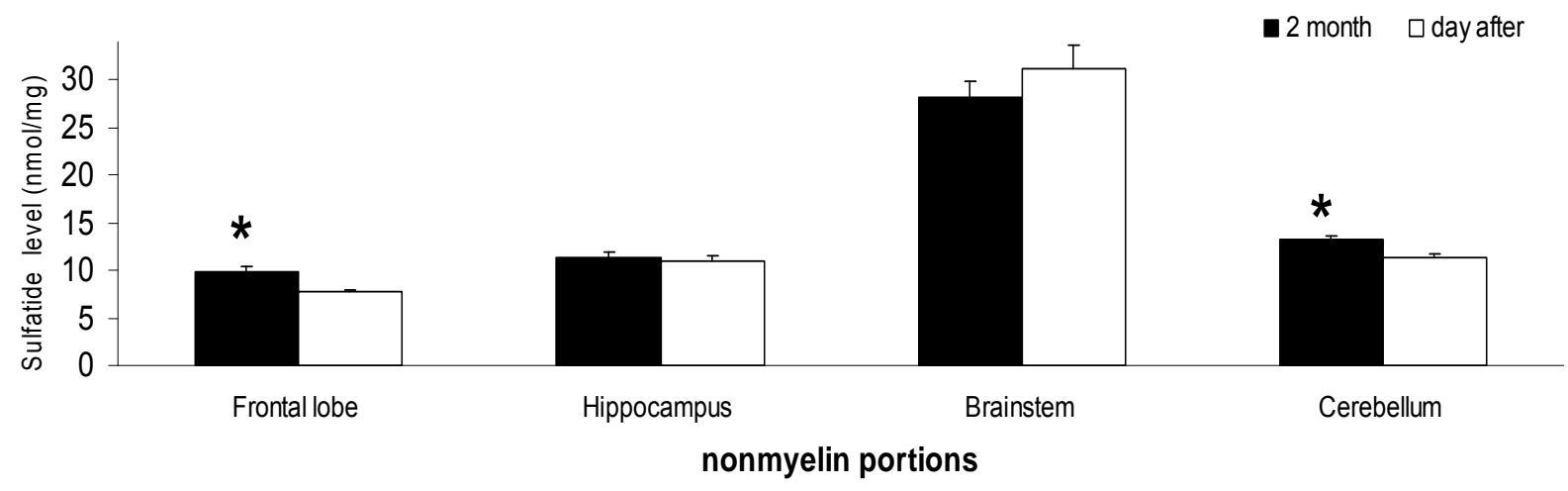

Figure 6.3. Sulfatide levels increase as a function of time.

A. Analysis of sulfatide levels in the myelin portions of the frontal lobe, hippocampus, brainstem, and cerebellum as a function of perfusion time revealed significant differences in the frontal lobe (n's = 12). B. Similarly, sulfatide levels in the nonmyelin portions as a function of time were significantly different in the frontal lobe as well as the cerebellum (n's $=12)$. 
Chapter 7

General Discussion 


\section{SUMMARY}

$\mathrm{AD}$ is a major health problem affecting about $5 \%$ of people age 65 years old and more than $35 \%$ of people over the age of 80 years old (Jaya Prasanthi et al., 2008). In addition over 100 million American adults have total blood cholesterol levels higher than $200 \mathrm{mg} / \mathrm{dL}$ and a fifth of those have cholesterol levels higher than $240 \mathrm{mg} / \mathrm{dL}$.

Concentrations of metals, especially copper, vary greatly in drinking water and have been linked to mental retardation and AD. Therefore, understanding the relationship between high dietary cholesterol, copper, learning and memory is important in light of continuing evidence of a strong link between these factors and AD. Furthermore, there is growing evidence that brain cholesterol, sulfatides and $\mathrm{A} \beta$ are involved in $\mathrm{AD}$ and their levels are impacted by dietary cholesterol. Developing a better understanding of how these factors are linked and their role in $\mathrm{AD}$ can help in better understanding this disease and one day helping to develop a cure.

In trying to understand the effect of cholesterol on learning, Schreurs in 2003 found that dietary cholesterol enhanced learning and increased the number of immunoreactive $A \beta$ neurons. However, a later study revealed that dietary cholesterol and the addition of copper supplemented water induced retardation in learning and the appearance of senile plaques. Our first study evaluated the effects of different concentrations of cholesterol and 0.12 ppm copper on learning and $A \beta$ levels. We found that learning was significantly greater in the highest cholesterol concentration fed to rabbits compared to controls. Furthermore, $A \beta$ staining showed a significant cholesterol concentration-dependent increase in the number of $A \beta$ positive neurons in the cortex of 
the cholesterol-fed rabbits. The data provided support for our previous finding that dietary cholesterol facilitates learning. This study again demonstrated that dietary cholesterol impacts learning and intracellular $A \beta$ levels. However, it also raised a question about continuing the use of copper- supplemented water in subsequent experiments. Examining these data in light of previous data, we see that the effect of dietary cholesterol with or without copper supplemented water yielded the same outcome: an increase in A $\beta$ immunoreactive neurons and enhanced learning. Only one experiment yielded a different outcome where the effect of dietary cholesterol with copper-supplemented water produced retarded learning and senile plaques. It remains to be determined the conditions under which plaques can be reliably obtained with dietary manipulations and the role that these plaques play in retarding learning.

To date, our observations about the effects of cholesterol on learning have been assessed during response acquisition i.e., the learning of a new memory. In our second study, we reported for the first time on the effect of a cholesterol diet on memory recall i.e., a previously formed memory. Rabbits were given trace conditioning of the NMR for ten days, then fed a $2 \%$ cholesterol diet for eight weeks, and then assessed for memory recall of the initially learned task. We showed that dietary cholesterol had an adverse effect on memory recall. Brain cholesterol and sulfatides play many important roles in learning and memory. So we also investigated whether dietary cholesterol caused an increase in brain cholesterol and sulfatide levels in four major brain structures (hippocampus, frontal lobe, brainstem, and cerebellum) using a technique for analyzing myelin and myelin-free (nonmyelin) fractions separately. Our data confirmed previous findings that dietary cholesterol does not directly affect cholesterol in the brain and 
established for the first time that it does not affect sulfatide levels in the brain. However both cholesterol and sulfatide levels did increase rather significantly in the hippocampus and frontal lobe as a function of learning and memory.

Our results from the second study were novel and interesting. This is to our knowledge the first time sulfatides have been studied in our cholesterol-fed model. Furthermore, the findings that brain cholesterol and sulfatide levels increase in learningand memory- related structures was very exciting and supports a role for both cholesterol and sulfatides in synaptic plasticity. At the same time, our study raises the issue of state dependency. Since the animals learned in a cholesterol-free state and then their memory retention was assessed in a cholesterol state, it could be argued that the animals can only recall the task in the same state in which the information was originally learned. However, the recovery of responding of the cholesterol fed animals as a function of repeated CS-alone presentations is similar to normal rabbits that show recovery of CRs as a function of repeated CS-alone presentations after much longer retention intervals - as long as six months after trace conditioning (Schreurs, 1998) and even nine months after delay conditioning (Schreurs, 1993) . One might speculate that dietary cholesterol slowed retrieval of the memory so that after a two-month period the cholesterol fed rabbits had the retrieval deficits of rabbits on a normal diet after six months. Importantly, we have shown that cholesterol-fed rabbits given CS-alone extinction immediately after tone-shock trace conditioning extinguish their CRs at the same rate and to the same level as normal diet controls (Schreurs et al., 2003).

Our last study was inspired by the findings that brain cholesterol and sulfatide levels were significantly increased in learning and memory related structures. In those 
experiments, the animals were exposed to dietary cholesterol; however our analysis revealed that the changes reported were independent of dietary manipulation. In addition the animals were exposed to several training paradigms (both trace conditioning and extinction) and all perfused after assessing memory recall. Given that these training paradigms are both hippocampal- and frontal-lobe dependent, in our third study we investigated whether the learning associated variations in brain cholesterol and sulfatide levels were a direct function of trace conditioning alone in the four major brain structures (hippocampus, frontal lobe, brainstem, and cerebellum) in the absence of a cholesterol diet. We found that trace conditioning alone did not produce any changes in sulfatide or cholesterol levels as a function of learning. Interestingly, a power analysis on the cholesterol and sulfatide levels as a function of pairings in the animals that equate the post-training duration with our last experiment indicated a lack of power in the myelin cholesterol levels in the hippocampus and a lack of effect in the nonmyelin cholesterol and sulfatide levels in the frontal lobe. Therefore, it maybe necessary to replicate our experiment to increase our sample sizes before making any final conclusions. We further investigated whether these changes in brain chemistry occurred a day after training or required the 9 weeks and two days, seen in the previous experiment. We found some significant changes in the sulfatide levels as a function of time, but not in the cholesterol levels. The data suggests that the changes we saw in sulfatide and cholesterol levels the previous experiment may require the combination of trace conditioning and extinction and that sulfatide level changes maybe time dependent. 


\section{FUTURE DIRECTIONS}

First, investigations are still needed to better understand the relationships between dietary cholesterol, copper, and A $\beta$. Specifically, trying to better understand the role copper plays in our model is important. The combination of cholesterol and copper in Sparks and Schreurs (2003) induced retardation in learning and the appearance of senile plaques. These plaques have not been repeated in our cholesterol-fed rabbit model. Recently, it was reported that dietary cholesterol and copper induced learning and memory impairments and the appearance of $A \beta$ plaques in mice (Lu et al., 2009). We have tried doubling the copper concentration from $0.12 \mathrm{ppm}$ to $0.24 \mathrm{ppm}$, however we were still unable to induced learning and memory impairments and the appearance of A $\beta$ plaques. Therefore, it would be worthwhile to revisit our own model and vary concentration of copper at higher levels than 0.24 ppm to try to replicate Spark and Schreurs' initial findings.

Second, examining the presented studies it is also clear that we need to examine a link between dietary cholesterol, memory, and A $\beta$. Presently, we are running an experiment in hopes of replicating the findings reported in our second study on the effects of dietary cholesterol on memory retention. In addition we are using different concentrations of cholesterol $(0,0.5,1.0$, and $2.0 \%)$ and plan to examine $A \beta$ levels in all the subjects. It would be interesting to report on any correlations between $A \beta$ levels and retardation in memory, especially since we believe that increases in $A \beta$ levels enhance learning as long as senile plaques are absent. One would be inclined to hypothesize that since we get retardation in memory we may see $A \beta$ plaques. The issue of state 
dependency should also be addressed by running a series of experiments alternating the dietary condition during the initial trace conditioning phase to be the same as the dietary condition during the extinction phase.

Third, follow up studies are needed on the relationship between learning and memory and brain levels of cholesterol and sulfatides. It would be important to replicate our findings reported in the last study (Chapter 6). The previous memory retention experiment (Chapter 5) had 12 subjects in the paired condition (collapsed across diet) and 12 in the unpaired (collapsed across diet) so we had essentially double the power to detect a pairing-specific change in cholesterol and sulfatide levels. A power analysis on Chapter 6 data indicates that the sample sizes in the cholesterol and sulfatides myelin portions of the hippocampus didn't have enough power to detect the differences. Moreover, it would be of interest to expand this study to include other membrane components such as phospholipids that have a role in learning and memory and to explore if they are affected by dietary cholesterol. 


\section{Reference List}

Abildayeva, K., Jansen, P. J., Hirsch-Reinshagen, V., Bloks, V. W., Bakker, A. H., Ramaekers, F. C. et al. (2006). 24(S)-hydroxycholesterol participates in a liver X receptor-controlled pathway in astrocytes that regulates apolipoprotein E-mediated cholesterol efflux. Journal of Biological Chemistry, 281, 12799-12808.

Alexander, G. J. \& Kopeloff, L. M. (1971). Induced hypercholesterolemia and decrease susceptibility to seizures in experimental animals. Experimental Neurology, 32, 134-140.

Alissa, E. M., Bahijri, S. M., Lamb, D. J., \& Ferns, G. A. A. (2004). The effects of coadministration of dietary copper and zinc supplements on atherosclerosis, antioxidant enzymes and indices of lipid peroxidation in the cholesterol-fed rabbit. International Journal of Experimental Pathology, 85, 265-275.

Als, H., Duffy, F. H., McAnulty, G. B., Rivkin, M. J., Vajapeyam, S., Mulkern, R. V. et al. (2004). Early experience alters brain function and structure. Pediatrics, 113, 846-857.

Ambrogini, P., Cuppini, R., Cuppini, C., Ciaroni, S., Cecchini, T., Ferri, P. et al. (2000). Spatial learning affects immature granule cell survival in adult rat dentate gyrus.

Neuroscience Letters, 286, 21-24.

Ambrogini, P., Orsini, L., Mancini, C., Ferri, P., Ciaroni, S., \& Cuppini, R. (2004).

Learning may reduce neurogenesis in adult rat dentate gyrus. Neuroscience Letters, 359, $13-16$.

Arispe, N., Rojas, E., \& Pollard, H. B. (1993). Alzheimer disease amyloid beta protein forms calcium channels in bilayer membranes: blockade by tromethamine and aluminum. Proc.Natl.Acad.Sci.U.S.A, 90, 567-571.

Atwood, C. S., Moir, R. D., Huang, X., Scarpa, R. C., Bacarra, N. M. E., Romano, D. M. et al. (1998). Dramatic aggregation of Alzheimer $\mathrm{A} \beta$ by $\mathrm{Cu}(\mathrm{II})$ is induced by conditions representing physiological acidosis. Journal of Biological Chemistry, 21, 12817-12826.

Atwood, C. S., Scarpa, R. C., Huang, X., Moir, R. D., Jones, W. D., Fairlie, D. P. et al. (2000). Characterization of copper interactions with Alzheimer amyloid $\beta$ peptides: identification of an attomolar-affinity copper binding site on amyloid $\beta 1-42$. Journal of Neurochemistry, 75, 1219-1233. 
Atzmon, G., Gabriely, I., Griener, W., Davidson, D., Schechter, C., \& Brazilai, N. (2002). Plasma HDL levels highly correlate with cognitive function in exceptional longevity. Journal of Gerontology Series A, Biological Sciences and Medical Sciences, 57A, M712-M715.

Bangasser, D. A., Waxler, D. E., Santollo, J., \& Shors, T. J. (2006). Trace Conditioning and the Hippocampus: The Importance of Contiguity. Journal of Neuroscience, 26, 87028706.

Bansal, R., Gard, A. L., \& Pfeiffer, S. E. (1988). Stimulation of oligodendrocyte differentiation in culture by growth in the presence of a monoclonal antibody to sulfated glycolipid. J.Neurosci.Res., 21, 260-267.

Bansal, R. \& Pfeiffer, S. E. (1989). Reversible inhibition of oligodendrocyte progenitor differentiation by a monoclonal antibody against surface galactolipids.

Proc.Natl.Acad.Sci.U.S.A, 86, 6181-6185.

Barghorn, S., Nimmrich, V., Striebinger, A., Krantz, C., Keller, P., Janson, B. et al. (2005). Globular amyloid beta-peptide oligomer - a homogenous and stable neuropathological protein in Alzheimer's disease. Journal of Neurochemistry, 95, 834847.

Barnea, A. \& Nottebohm, F. (1994). Seasonal recruitment of hippocampal neurons in adult free-ranging black-capped chickadees. Proc.Natl.Acad.Sci.U.S.A, 91, 11217-11221.

Barrientos, R. M., Higgins, E. A., Biedenkapp, J. C., Sprunger, D. B., Wright-Hardesty, K. J., Watkins, L. R. et al. (2006). Peripheral infection and aging interact to impair hippocampal memory consolidation. Neurobiology of Aging, 27, 723-732.

Bartzokis, G., Beckson, M., Lu, P. H., Nuechterlein, K. H., Edwards, N., \& Mintz, J. (2001). Age-Related Changes in Frontal and Temporal Lobe Volumes in Men: A Magnetic Resonance Imaging Study. Archives of General Psychiatry, 58, 461-465.

Beaslely, C. L., Honer, W. G., von Bergmann, K., Falkai, P., Lutjohann, D., \& Bayer, T. A. (2005). Reductions in cholesterol and synaptic markers in association cortex in mood disorders. Bipolar Disorder, 7, 449-455.

Bellingham, S. A., Lahiri, D. K., Maloney, B., La Fontaine, S., Multhaup, G., \& Camakaris, J. (2004). Copper depletion down-regulates expression of the Alzheimer's 
disease amyloid- $\beta$ precursor protein gene. The Journal of Biological Chemsitry, 279, 20378-20386.

Bengtsson, S. L., Nagy, Z., Skare, S., Forsman, L., Forssberg, H., \& Ullen, F. (2005). Extensive piano practicing has regionally specific effects on white matter development. Nature Neuroscience, 8, 1148-1150.

Berger, T. W., Bassett, J. L., \& Orr, W. B. (1991). Multiple memory systems of the mammalian brain involved in classical conditioning. In (pp. 271-310).

Berger, T. W. \& Orr, W. B. (1983). Hippocampectomy selectively disrupts discrimination reversal conditioning of the rabbit nictitating membrane response. Behavioural Brain Research, 8, 49-68.

Berkman, Z., Tanriover, G., Acar, G., Sati, L., Altug, T., \& Demir, R. (2009). Changes in the brain cortex of rabbits on a cholesterol-rich diet following supplementation with a herbal extract of Tribulus terrestris. Histol.Histopathol., 24, 683-692.

Berntson, Z., Hansson, E., Ronnback, L., \& Fredman, P. (1998). Intracellular sulfatide expression in a subpopulation of astrocytes in primary cultures. J.Neurosci.Res., 52, 559568.

Beylin, A. V., Gandhi, C. C., Wood, G. E., Talk, A. C., Matzel, L. D., \& Shors, T. J. (2001). The role of the hippocampus in trace conditioning: temporal discontinuity or task difficulty. Neurobiology of Learning and Memory, 76, 447-461.

Bird, K. D. (2004). Analysis of Variance via Confience Intervals. London: Sage.

Bjorkhem, I., Lutjohann, D., Diczfalusy, U., Stahle, L., Ahlskog, J. E., \& Ahlborg, G. (1998). Cholesterol homeostasis in human brain: turnover of 24S-hydroxycholesterol and evidence for a cerebral origin of this oxysterol in the circulation. Journal of Lipid Research, 39, 1594-1600.

Bjorkhem, I. \& Meaney, S. (2004). Brain cholesterol: long secret life behind a barrier. Arteriosclerosis, Thrombosis and Vascular Biology, 24, 1-11.

Bocan, T. M. A., Mueller, S. B., Brown, E. Q., Lee, P., Bocan, M. J., Rea, T. et al. (1998). HMG-CoA reductase and ACAT inhibitors act synergistically to lower plasma 
cholesterol and limit atherosclerosis lesion development in the cholesterol-fed rabbit. Atherosclerosis, 139, 21-30.

Boleman, S. L., Graf, T. L., Mersmann, H. J., Su, D. R., Krook, L. P., Savell, J. W. et al. (1998). Pigs fed cholesterol neonatally have increased cerebrum cholesterol as young adults. Journal of Nutrition, 128, 2498-2504.

Brennan, F. X., Beck, K. D., \& Servatius, R. J. (2004). Proinflammatory cytokines differentially affect leverpress avoidance acquisition in rats. Behavioural Brain Research, 153, 351-355.

Brennan, F. X. \& Tieder, J. B. I. (2006). Centrally administered tumor necrosis tactor- $\alpha$ facilitates the avoidance performance of Sprague-Dawley rats. Brain Res., 1109, $142-$ 145.

Bretillon, L., Lutjohann, D., Stahle, L., Widhe, T., Bindl, L., Eggertsen, G. et al. (2000). Plasma levels of 24S-hydroxycholesterol reflect the balance between cerebral production and hepatic metabolism and are inversely related to body surface. Journal of Lipid Research, 41, 840-845.

Bu, G. (2009). Apolipoprotein E and its receptors in Alzheimer's disease: pathways, pathogenesis and therapy. Nat.Rev.Neurosci., 10, 333-344.

Buck, D. L., Seager, M. A., \& Schreurs, B. G. (2001). Conditioning-specific reflex modification of the rabbit (Oryctolagus cuniculus) nictitating membrane response: generality and nature of the phenomenon. Behavioral Neuroscience, 115, 1039-1047.

Bush, A. I. (2002). Metal complexing agents as therapies for Alzheimer's disease. Neurobiology of Aging, 23, 1031-1038.

Bush, A. I., Pettingell, W. H., Multhaup, G., Paradis, M., Vonsattel, J. P., Gusella, J. F. et al. (1994a). Rapid induction of Alzheimer A beta amyloid formation by zinc. Science, 265, 1464-1467.

Bush, A. I., Pettingell, W. H., Jr., Paradis, M. D., \& Tanzi, R. E. (1994b). Modulation of A beta adhesiveness and secretase site cleavage by zinc. Journal of Biological Chemistry, 269, 12152-12158. 
Butterfield, D. A., Galvan, V., Lange, M. B., Tang, H., Sowell, R. A., Spilman, P. et al. (2010). In vivo oxidative stress in brain of Alzheimer disease transgenic mice:

Requirement for methionine 35 in amyloid beta-peptide of APP. Free Radic.Biol.Med., 48, 136-144.

Buxbaum, J. D., Cullen, E. I., \& Friedhoff, L. T. (2002). Pharmacological concentrations of the HMG-CoA reductase inhibitor lovastatin decrease the formation of the Alzheimer's $\beta$-amyloid peptide in vitro and in patients. Frontiers in Bioscience, 7, a50-a59.

Caille, I., Allinquant, B., Dupont, E., Bouillot, C., Langer, A., Muller, U. et al. (2004). Soluble form of amyloid precursor protein regulates proliferation of progenitors in the adult subventricular zone. Development, 131, 2173-2181.

Cammarota, M., Bevilaqua, L. R., Barros, D. M., Vianna, M. R., Izquierdo, L. A., Medina, J. H. et al. (2005). Retrieval and the extinction of memory. Cell Mol.Neurobiol., 25, 465-474.

Canevari, L. \& Clark, J. B. (2007). Alzheimer's disease and cholesterol: the fat connection. Neurochemical Research, 32, 739-750.

Cao, D., Fukuchi, K., Wan, H., Kim, H., \& Li, L. (2006). Lack of LDL receptor aggravates learning deficits and amyloid deposits in Alzheimer transgenic mice. Neurobiology of Aging, 27, 1632-1643.

Cao, X. \& Sudhof, T. C. (2001). A transcriptionally [correction of transcriptively] active complex of APP with Fe65 and histone acetyltransferase Tip60. Science, 293, 115-120.

Cavallaro, S., Schreurs, B. G., Zhao, W., D'Agata, V., \& Alkon, D. L. (2001). Gene expression profiles during long-term memory consolidation. European Journal of Neuroscience, 13, 1-8.

Cheng, H., Xu, J., McKeel, D. W., Jr., \& Han, X. (2003). Specificity and potential mechanism of sulfatide deficiency in Alzheimer's disease: an electrospray ionization mass spectrometric study. Cell Mol.Biol.(Noisy.-le-grand), 49, 809-818.

Cheng, H., Zhou, Y., Holtzman, D. M., \& Han, X. (2008). Apolipoprotein E mediates sulfatide depletion in animal models of Alzheimer's disease. Neurobiol.Aging. 
Cherny, R. A., Atwood, C. S., Xilinas, M. E., Gray, D. N., Jones, W. D., McLean, C. A. et al. (2001). Treatment with a copper-zinc chelator markedly and rapidly inhibits $\beta$ amyloid accumulation in Alzheimer's disease transgenic mice. Neuron, 30, 665-676.

Chi, S. \& Qi, Z. (2006). Regulatory effect of sulphatides on BKCa channels. Br J Pharmacol, 149, 1031-1038.

Cleary, J. P., Walsh, D. M., Hofmeister, J. J., Shankar, G. M., Kuskowski, M. A., Selkoe, D. J. et al. (2005). Natural oligomers of the amyloid- $\beta$ protein specifically disrupt cognitive function. Nature Neuroscience, 8, 79-84.

Coleman, S. R. \& Gormezano, I. (1971). Classical conditioning of the rabbit's (Oryctolagus cuniculus) nictitating membrane response under symmetrical CS-US interval shifts. Journal of Comparative and Physiological Psychology, 77, 447-455.

Cornett, C. R., Markesbery, W. R., \& Ehmann, W. D. (1998). Imbalances of trace elements related to oxidative damage in Alzheimer's disease brain. NeuroToxicology, 19, 339-346.

Cui, R., Iso, H., Toyoshima, H., Date, C., Yamamoto, A., Kikuchi, S. et al. (2006). Serum cholesterol levels of mortality from stroke and coronary heart disease in Japanese: The JACC study. Atherosclerosis, In press, x.

Curtain, C. C., Ali, F., Volitakis, I., Cherny, R. A., Norton, R. S., Beyreuther, K. et al. (2001). Alzheimer's disease amyloid- $\beta$ binds copper and zinc to generate an allosterically ordered membrane-penetrating structure containing superoxide dismutase-like subunits. Journal of Biological Chemistry, 276, 20466-20473.

D'Hooge, R., Hartmann, D., Manil, J., Colin, F., Gieselmann, V., \& De Deyn, P. P. (1999). Neuromotor alterations and cerebellar deficits in aged arylsulfatase A-deficient transgenic mice. Neuroscience Letters, 273, 93-96.

D'Hooge, R., Van, D. D., Franck, F., Gieselmann, V., \& De Deyn, P. P. (2001). Hyperactivity, neuromotor defects, and impaired learning and memory in a mouse model for metachromatic leukodystrophy. Brain Research, 907, 35-43.

Dahlgren, K. N., Manelli, A. M., Stine, Jr. W. B., Baker, L. K., Krafft, G. A., \& Ladu, M. J. (2002). Oligomeric and fibrillar species of amyloid- $\beta$ peptides differentially affect neuronal viability. Journal of Biological Chemistry, 35, 32046-32053. 
Danik, M., Champagne, D., Petit-Turcotte, C., Beffert, U., \& Poirier, J. (1999). Brain lipoprotein metabolism and its relation to neurodegenerative disease. Critical Reviews in Neurobiology, 13, 357-407.

Davies, L., Wolska, B., Hilbich, C., Multhaup, G., Martins, R., Simms, G. et al. (1988). A4 amyloid protein deposition and the diagnosis of Alzheimer's disease: prevalence in aged brains determined by immunocytochemistry compared with conventional neuropathologic techniques. Neurology, 38, 1688-1693.

Deaux, E. B. \& Gormezano, I. (1963). Eyeball retraction: classical conditioning and extinction in the albino rabbit. Science, 141, 630-631.

Desplats, P. A., Denny, C. A., Kass, K. E., Gilmartin, T., Head, S. R., Sutcliffe, J. G. et al. (2007). Glycolipid and ganglioside metabolism imbalances in Huntington's disease. Neurobiol.Dis., 27, 265-277.

Deyo, R. A., Straube, K. T., \& Disterhoft, J. F. (1989). Nimodipine facilitates associative learning in aging rabbits. Science, 243, 809-811.

Dietschy, J. M. (2009). Central nervous system: cholesterol turnover, brain development and neurodegeneration. Biol.Chem., 390, 287-293.

Dietschy, J. M. \& Turley, S. D. (2001). Cholesterol metabolism in the brain. Current Opinion in Lipidology, 12, 105-112.

Dietschy, J. M. \& Turley, S. D. (2004). Cholesterol metabolism in the central nervous system during early development and in the mature animal. Journal of Lipid Research, $45,1375-1397$.

Dobrossy, M. D., Drapeau, E., Aurousseau, C., Le, M. M., Piazza, P. V., \& Abrous, D. N. (2003). Differential effects of learning on neurogenesis: learning increases or decreases the number of newly born cells depending on their birth date. Mol.Psychiatry, 8, 974-982.

Drapeau, E., Mayo, W., Aurousseau, C., Le, M. M., Piazza, P. V., \& Abrous, D. N. (2003). Spatial memory performances of aged rats in the water maze predict levels of hippocampal neurogenesis. Proc.Natl.Acad.Sci.U.S.A, 100, 14385-14390. 
Dufour, F., Liu, Q.-Y., Gusev, P., Alkon, D. L., \& Atzori, M. (2006). Cholesterolenriched diet affects spatial learning and synaptic function in hippocampal synapses. Brain Research, 1103, 88-98.

Eckhardt, M. (2008). The role and metabolism of sulfatide in the nervous system. Mol.Neurobiol., 37, 93-103.

Eckhardt, M., Hedayati, K. K., Pitsch, J., Lullmann-Rauch, R., Beck, H., Fewou, S. N. et al. (2007). Sulfatide storage in neurons causes hyperexcitability and axonal degeneration in a mouse model of metachromatic leukodystrophy. Journal of Neuroscience, 27, 90099021.

Elias, P. K., Elias, M. F., D'Agostino, R. B., Sullivan, L. M., \& Wolf, P. A. (2005). Serum cholesterol and cognitive performance in the Framingham heart study. Psychosomatic Medicine, 67, 24-30.

Endo, Y., Nishimura, J.-I., \& Kimura, F. (1996). Impairment of maze learning in rats following long-term glucocorticoid treatments. Neuroscience Letters, 203, 199-202.

Evans, R. M., Emsley, C. L., Gao, S., Sahota, A., Hall, K. S., Farlow, M. R. et al. (2000). Serum cholesterol, $A P O E$ genotype, and the risk of Alzheimer's disease: a populationbased study of African Americans. Neurology, 54, 240-242.

Fassbender, K., Simons, M., Bergmann, C., Stroick, M., Lutjohann, D., Keller, P. et al. (2001). Simvastatin strongly reduces levels of Alzheimer's disease $\beta$-amyloid peptides $\mathrm{A} \beta 42$ and $\mathrm{A} \beta 40$ in vitro and in vivo. Proceedings of the National Academy of Sciences, USA, 98, 5856-5861.

Faulks, S. C., Turner, N., Else, P. L., \& Hulbert, A. J. (2006). Calorie Restriction in Mice: Effects on Body Composition, Daily Activity, Metabolic Rate, Mitochondrial Reactive Oxygen Species Production, and Membrane Fatty Acid Composition. Journals of Gerontology Series A: Biological Sciences and Medical Sciences, 61, 781-794.

Fazio, S. \& Linton, M. F. (2001). The inflamed plaque: cytokine production and cellular cholesterol balance in the vessel wall. American Journal of Cardiology, 88A, 12E-15E.

Finking, G. \& Hanke, H. (1997). Nikolaj Nicolajewisch Anitschkow (1885-1964) established the cholesterol-fed rabbit as a model for atherosclerosis research. Atherosclerosis, 135, 1-7. 
Foster, T. C. (2006). Biological markers of age-related memory deficits. Treatment of senescent physiology. CNS Drugs, 20, 153-166.

Frank, C., Rufini, S., Tamcredi, V., Forcina, R., Grossi, D., \& D'Arcangelo, G. (2008). Cholesterol depletion inhibits synaptic transmission and synaptic plasticity in rat hippocampus. Experimental Neurology, 212, 407-414.

Frankland, P. W. \& Bontempi, B. (2005). The organization of recent and remote memories. Nature Reviews Neuroscience, 6, 119-130.

Frederickson, C. J. (1989). Neurobiology of zinc and zinc-containing neurons. Int.Rev.Neurobiol., 31, 145-238.

Freir, D. B., Fedriani, R., Scully, D., Smith, I. M., Selkoe, D. J., Walsh, D. M. et al. (2010). Abeta oligomers inhibit synapse remodelling necessary for memory consolidation. Neurobiology of Aging.

Gandy, S. (2005). The role of cerebral amyloid $\beta$ accumulation in common forms of Alzheimer's disease. Journal of Clinical Investigation, 11, 1121-1129.

Ghribi, O., Golovko, M. Y., Larsen, B., Schrag, M., \& Murphy, E. J. (2006a). Deposition of iron and $\beta$-amyloid plaques is associated with cortical cellular damage in rabbits fed with long-term cholesterol-enriched diets. Journal of Neurochemistry, 99, 438-449.

Ghribi, O., Larsen, B., Schrag, M., \& Herman, M. M. (2006b). High cholesterol content in neurons increases BACE, $\beta$-amyloid, and phosphorylated tau levels in rabbit hippocampus. Experimental Neurology, 200, 460-467.

Giese, K. P., Storm, J. F., Reuter, D., Fedorov, N. B., Shao, L.-R., Leichter, T. et al. (1998). Reduced $\mathrm{K}^{+}$channel inactivation, spike broadening, and after-hyperpolarization in Kvß1.1-deficient mice with impaired learning. Learning \& Memory, 5, 257-273.

Glenner, G. G. \& Wong, C. W. (1984a). Alzheimer's disease and Down's syndrome: sharing of a unique cerebrovascular amyloid fibril protein.

Biochem.Biophys.Res.Commun., 122, 1131-1135.

Glenner, G. G. \& Wong, C. W. (1984b). Alzheimer's disease: initial report of the purification and characterization of a novel cerebrovascular amyloid protein. Biochem.Biophys.Res.Commun., 120, 885-890. 
Goldman, S. A. \& Nottebohm, F. (1983). Neuronal production, migration, and differentiation in a vocal control nucleus of the adult female canary brain.

Proc.Natl.Acad.Sci.U.S.A, 80, 2390-2394.

Goritz, C., Mauch, D. H., Nagler, K., \& Pfrieger, F. W. (2002). Role of glial-derived cholesterol in synaptogenesis: new revelations in the synapse-glia affair. Journal of Physiology (Paris), 96, 257-263.

Gormezano, I. (1966). Classical conditioning. In J.B.Sidowski (Ed.), Experimental methods and instrumentation in psychology (pp. 385-420). New York: McGraw-Hill.

Gormezano, I. \& Gibbs, C. M. (1988). Transduction of the rabbit's nictitating membrane response. Behavior Research Methods, Instruments, \& Computers, 20, 18-21.

Gormezano, I., Schneiderman, N., Deaux, E. G., \& Fuentes, I. (1962). Nictitating membrane: Classical conditioning and extinction in the albino rabbit. Science, 138, 3334.

Gould, E., Beylin, A., Tanapat, P., Reeves, A., \& Shors, T. J. (1999). Learning enhances adult neurogenesis in the hippocampal formation. Nature Neuroscience, 2, 260-265.

Green, J. T. \& Arenos, J. D. (2007). Hippocampal and cerebellar single-unit activity during delay and trace eyeblink conditioning in the rat. Neurobiology of Learning and Memory, 87, 269-284.

Gruart, A., Morcuende, S., Martinez, S., \& Delgado-Garcia, J. M. (2000). Involvement of cerebral cortical structures in the classical conditioning of eyelid responses in rabbits. Neuroscience, 100, 719-730.

Guardia-Laguarta, C., Coma, M., Pera, M., Clarimon, J., Sereno, L., Agullo, J. M. et al. (2009). Mild cholesterol depletion reduces amyloid-beta production by impairing APP trafficking to the cell surface. J.Neurochem., 110, 220-230.

Guo, J., Chi, S., Xu, H., Jin, G., \& Qi, Z. (2008). Effects of cholesterol levels on the excitability of rat hippocampal neurons. Molecular Membrane Biology, 25, 216-223.

Hairston, I. S., Little, M. T., Scanlon, M. D., Barakat, M. T., Palmer, T. D., Sapolsky, R. M. et al. (2005). Sleep restriction suppresses neurogenesis induced by hippocampusdependent learning. J.Neurophysiol., 94, 4224-4233. 
Han, X. (2007). Potential mechanisms contributing to sulfatide depletion at the earliest clinically recognizable stage of Alzheimer's disease: a tale of shotgun lipidomics. Journal of Neurochemistry, 103 Suppl 1, 171-179.

Han, X., Cheng, H., Fryer, J. D., Fagan, A. M., \& Holtzman, D. M. (2003a). Novel role for apolipoprotein $\mathrm{E}$ in the central nervous system. Modulation of sulfatide content. Journal of Biological Chemistry, 278, 8043-8051.

Han, X., Fagan, A. M., Cheng, H., Morris, J. C., Xiong, C., \& Holtzman, D. M. (2003b). Cerebrospinal fluid sulfatide is decreased in subjects with incipient dementia. Annals of Neurology, 54, 115-119.

Han, X., Holtzman, M., McKeel, D. W., Jr., Kelley, J., \& Morris, J. C. (2002).

Substantial sulfatide deficiency and ceramide elevation in very early Alzheimer's disease: potential role in disease pathogenesis. Journal of Neurochemistry, 82, 809-818.

Hartmann, T. (2001). Cholesterol, A $\beta$ and Alzheimer's disease. Trends in Neurosciences, $11, \mathrm{~S} 45-\mathrm{S} 48$.

Hayashi, H., Igbavboa, U., Hamanaka, H., Kobayashi, M., Fujita, S. C., Wood, W. G. et al. (2002). Cholesterol is increased in the exofacial leaflet of synaptic plasma membranes of human apolipoprotein E4 knock-in mice. NeuroReport, 13, 383-386.

Henry, P. D. \& Bentley, K. I. (1981). Suppression of atherogenesis in cholesterol-fed rabbit treated with nifedipine. Journal of Clinical Investigation, 68, 1366-1369.

Herz, J. \& Chen, Y. (2006). Reelin, lipoprotein receptors and synaptic plasticity. Nature Reviews Neuroscience, 7, 850-859.

Heverin, M., Meaney, S., Lutjohann, D., Diczfalusy, U., Wahren, J., \& Bjorkhem, I. (2005). Crossing the barrier: net flux of 27-hydroxycholesterol into the human brain. Journal of Lipid Research, 46, 1047-1052.

Hoglund, K., Thelen, K. M., Syversen, S., Sjogren, M., von Bergmann, K., Wallin, A. et al. (2005). The effect of simvastatin treatment on the amyloid precursor protein and brain cholesterol metabolism in patients with Alzheimer's disease. Dementia and Geriatric Cognitive Disorders, 19, 256-265. 
Hojo, Y., Murakami, G., Mukai, H., Higo, S., Hatanaka, Y., Ogiue-Ikeda, M. et al. (2008). Estrogen synthesis in the brain--role in synaptic plasticity and memory. Mol.Cell Endocrinol., 290, 31-43.

Holmes, M. M., Wide, J. K., \& Galea, L. A. (2002). Low levels of estradiol facilitate, whereas high levels of estradiol impair, working memory performance on the radial arm maze. Behavioral Neuroscience, 116, 928-934.

Honke, K., Hirahara, Y., Dupree, J., Suzuki, K., Popko, B., Fukushima, K. et al. (2002). Paranodal junction formation and spermatogenesis require sulfoglycolipids. Proceedings of the National Academy of Sciences of the United States of America, 99, 4227-4232.

Hoyer, S. \& Riederer, P. (2007). Alzheimer disease - no traget for statin treatment. A mini review. Neurochem.Res., 32, 695-706.

Huang, X., Atwood, C. S., Moir, R. D., Hartshorn, M. A., Tanzi, R. E., \& Bush, A. I. (2004a). Trace metal contamination initiates the apparent auto-aggregation, amyloidosis, and oligomerization of Alzheimer's A $\beta$ peptides. Journal of Biological Inorganic Chemistry, 9, 954-960.

Huang, X., Cuajungco, M. P., Atwood, C. S., Hartshorn, M. A., Tyndall, J. D., Hanson, G. R. et al. (1999). Cu(II) potentiation of alzheimer abeta neurotoxicity. Correlation with cell-free hydrogen peroxide production and metal reduction. J.Biol.Chem., 274, 3711137116.

Huang, Y., Walker, K. E., Hanley, F., Narula, J., Houser, S. R., \& Tulenko, T. N. (2004b). Cardiac systolic and diastolic dysfunction after a cholesterol-rich diet. Circulation, 109, 97-102.

Hung, Y. H., Bush, A. I., \& Cherny, R. A. (2010). Copper in the brain and Alzheimer's disease. J.Biol.Inorg.Chem., 15, 61-76.

Ikonen, E. (2006). Mechanisms for cellular cholesterol transport: defects and human disease. Physiological Reviews, 86, 1237-1261.

Inda, M. C., Delgado-Garcia, J. M., \& Carrion, A. M. (2005). Acquisition, consolidation, reconsolidation, and extinction of eyelid conditioning responses require de novo protein synthesis. The Journal of Neuroscience, 25, 2070-2080. 
Isaac, G., Pernber, Z., Gieselmann, V., Hansson, E., Bergquist, J., \& Mansson, J. E. (2006). Sulfatide with short fatty acid dominates in astrocytes and neurons. FEBS J., 273, 1782-1790.

Ishibashi, T., Dupree, J. L., Ikenaka, K., Hirahara, Y., Honke, K., Peles, E. et al. (2002). A myelin galactolipid, sulfatide, is essential for maintenance of ion channels on myelinated axon but not essential for initial cluster formation. Journal of Neuroscience, $22,6507-6514$.

Izquierdo, L. A., Barros, D. M., da Costa, J. C., Furini, C., Zinn, C., Cammarota, M. et al. (2007). A link between role of two prefrontal areas in immediate memory and in longterm memory consolidation. Neurobiology of Learning and Memory, 88, 160-166.

Jarvik, G. P., Wijsman, E. M., Kukull, W. A., Schellenberg, G. D., Yu, C., \& Larson, E. B. (1995). Interactions of apolipoprotein E genotype, total cholesterol level, age, and sex in prediction of Alzheimer's disease. Neurology, 45, 1092-1096.

Jaya Prasanthi, R. P., Schommer, E., Thomasson, S., Thompson, A., Feist, G., \& Ghribi, O. (2008). Regulation of $\beta$-amyloid levels in the brain of cholesterol-fed rabbit, a model system for sporadic Alzheimer's disease. Mechanisms of Ageing and Development, 29, 649-655.

Johnston, D., Christie, B. R., Frick, A., Gray, R., Hoffman, D. A., Schexnayder, L. K. et al. (2003). Active dendrites, potassium channels and synaptic plasticity. Philosophical Transactions of the Royal Society London B, 358, 667-674.

Kamal, A., menar-Queralt, A., LeBlanc, J. F., Roberts, E. A., \& Goldstein, L. S. (2001). Kinesin-mediated axonal transport of a membrane compartment containing beta-secretase and presenilin-1 requires APP. Nature, 414, 643-648.

Kamenetz, F., Tomita, T., Hsieh, H., Seabrook, G. R., Borchelt, D. R., Iwatsubo, T. et al. (2003). APP processing and synaptic function. Neuron, 37, 925-937.

Kane, K. A. \& Robinson, G. B. (1999). Effect of chronic nimodipine on spatial learning and on long-term potentiation. Behavioural Brain Research, 98, 95-101.

Kang, J., Lemaire, H. G., Unterbeck, A., Salbaum, J. M., Masters, C. L., Grzeschik, K. H. et al. (1987). The precursor of Alzheimer's disease amyloid A4 protein resembles a cellsurface receptor. Nature, 325, 733-736. 
Kaul, D. (2001). Molecular link between cholesterol, cytokines and atherosclerosis. Molecular and Cellular Neuroscience, 219, 65-71.

Keshet, B., Yang, I. H., \& Good, T. A. (2010). Can size alone explain some of the differences in toxicity between beta-amyloid oligomers and fibrils? Biotechnol.Bioeng..

Kessler, A. R., Kessler, B., \& Yehuda, S. (1986). In vivo modulation of brain cholesterol level and learning performance by a novel plant lipid: indication of interactions between hippocampal-cortical cholesterol and learning. Life Sciences, 38, 1185-1192.

Kim, J. J., Clark, R. E., \& Thompson, R. F. (1995). Hippocampectomy impairs the memory of recently, but not remotely, acquired trace eyeblink conditioned responses. Behavioral Neuroscience, 109, 195-203.

Kivipelto, M., Helkala, E.-L., Hanninen, T., Laakso, M. P., Hallikainen, M., Alhainen, K. et al. (2001). Midlife vascular risk factors and late-life mild cognitive impairment: a population-based study. Neurology, 56, 1683-1689.

Kojro, E. \& Fahrenholz, F. (2005). The non-amyloidogenic pathway: structure and function of alpha-secretases. Subcell.Biochem., 38, 105-127.

Kolsch, H., Heun, R., Kerksiek, A., Bergmann, K. V., Maier, W., \& Lutjohann, D. (2004). Altered levels of plasma 24S- and 27-hydroxycholesterol in demented patients. Neuroscience Letters, 368, 303-308.

Kolsch, H., Lutjohann, D., von Bergmann, K., \& Heun, R. (2003). The role of 24Shydroxycholesterol in Alzheimer's disease. Journal of Nutrition, Health and Aging, 7, 3741.

Konat, G. (1981). Intracellular translocation of newly synthesized myelin proteins in the rat brain stem slices. Experimental Neurology, 73, 254-266.

Korade, Z., Mi, Z., Portugal, C., \& Schor, N. F. (2007). Expression and p75 neurotrophin receptor dependence of cholesterol synthetic enzymes in adult mouse brain. Neurobiology of Aging, 28, 1522-1531.

Koudinov, A. R. \& Koudinova, N. V. (2001). Essential role of cholesterol in synaptic plasticity and neuronal degeneration. FASEB Journal, 15, 1858-1860. 
Koudinov, A. R. \& Koudinova, N. V. (2003). Cholesterol, synaptic function and Alzheimer's disease. Pharmacopsychiatry, 36, S107-S112.

Koudinov, A. R. \& Koudinova, N. V. (2005). Cholesterol homeostasis failure as a unifying cause of synaptic degeneration. Journal of the Neurological Sciences, 229-230, 233-240.

Koudinov, A. R., Koudinova, N. V., Kumar, A., Beavis, R. C., \& Ghiso, J. (1996). Biochemical characterization of Alzheimer's soluble amyloid beta protein in human cerebrospinal fluid: association with high density lipoproteins.

Biochem.Biophys.Res.Commun., 223, 592-597.

Lamb, D. J., Avades, T. Y., \& Ferns, G. A. A. (2001). Biphasic modulation of atherosclerosis induced by graded dietary copper supplementation in the cholesterol-fed rabbit. International Journal of Experimental Pathology, 82, 287-294.

Ledesma, M. D. \& Dotti, C. G. (2006). Amyloid excess in Alzheimer's disease: what is cholesterol to be blamed for? FEBS Letters, 580, 5525-5532.

Lemaire, V., Koehl, M., Le, M. M., \& Abrous, D. N. (2000). Prenatal stress produces learning deficits associated with an inhibition of neurogenesis in the hippocampus. Proc.Natl.Acad.Sci.U.S.A, 97, 11032-11037.

Leoni, V., Masterman, T., Patel, P., Meaney, S., Diczfalusy, U., \& Bjorkhem, I. (2003). Side chain oxidized oxysterols in cerebrospinal fluid and the integrity of blood-brain and blood-cerebrospinal fluid barriers. Journal of Lipid Research, 44, 793-799.

Lesser, G. T., Haroutunian, V., Purohit, D. P., Beeri, M. S., Schmeidler, J., Honkanen, L. et al. (2009). Serum lipid levels are related to Alzheimer's pathology in nursing home residents. Dementia and Geriatric Cognitive Disorders, 27, 42-49.

Leuner, B., Mendolia-Loffredo, S., Kozorovitskiy, Y., Samburg, D., Gould, E., \& Shors, T. J. (2004). Learning enhances the survival of new neurons beyond the time when the hippocampus is required for memory. Journal of Neuroscience, 24, 7477-7481.

Li, L., Cao, D., Kim, H., Lester, R., \& Fukuchi, K. (2006). Simvastatin enhances learning and memory independent of amyloid load in mice. Annals of Neurology, 60, 729-739. 
Liu, J. P., Tang, Y., Zhou, S., Toh, B. H., McLean, C., \& Li, H. (2009). Cholesterol involvement in the pathogenesis of neurodegenerative diseases. Mol.Cell Neurosci..

Liu, S. T., Howlett, G., \& Barrow, C. J. (1999). Histidine-13 is a crucial residue in the zinc ion-induced aggregation of the A beta peptide of Alzheimer's disease. Biochemistry, 38, 9373-9378.

Locatelli, S., Lutjohann, D., Schmidt, H. H.-J., Otto, C., Beisiegel, U., \& von Bergmann, K. (2002). Reduction of plasma 24S-hydroxycholesterol (cerebrosterol) levels using high-dosage simvastatin in patients with hypercholesterolemia. Evidence that simvastatin affects cholesterol metabolism in the human brain. Archives of Neurology, 59, 213-216.

Lovell, M. A., Robertson, J. D., Teesdale, W. J., Campbell, J. L., \& Markesbery, W. R. (1998). Copper, iron and zinc in Alzheimer's disease senile plaques. Journal of Neurological Sciences, 158, 47-52.

Lovstad, R. A. (1987). Copper catalyzed oxidation of ascorbate (vitamin C). Inhibitory effect of catalase, superoxide dismutase, serum proteins (ceruloplasmin, albumin, apotransferrin) and amino acids. Int.J.Biochem., 19, 309-313.

Lu, J., Wu, D.-M., Zheng, Y.-L., Sun, D.-X., Hu, B., Shan, Q. et al. (2009). Trace amounts of copper exacerbate beta amyloid-induced neurotoxicity in the cholesterol-fed mice through TNF-mediated inflammatory pathway. Brain, Behavior, and Immunity, 23, 193-203.

Lublin, A. L. \& Gandy, S. (2010). Amyloid-beta oligomers: possible roles as key neurotoxins in Alzheimer's Disease. Mt.Sinai J.Med., 77, 43-49.

Luine, V. N. (2007). The prefrontal cortex, gonadal hormones and memory. Horm.Behav., 51, 181-182.

Luine, V. N., Richards, S. T., Wu, V. Y., \& Beck, K. D. (1998). Estradiol enhances learning and memory in a spatial memory task and effects levels of monoaminergic neurotransmitters. Horm.Behav., 34, 149-162.

Lund, E. G., Guileyardo, J. M., \& Russell, D. W. (1999). cDNA cloning of cholesterol 24-hydroxylase, a mediator of cholesterol homeostasis in the brain. Proceedings of the National Academy of Sciences, USA, 96, 7238-7243. 
Lutjohann, D., Breuer, O., Ahlboorg, G., Nennesmo, I., Siden, A., Diczfalusy, U. et al. (1996). Cholesterol homeostasis in human brain: evidence for an age-dependent flux of 24S-hydroxycholesterol from the brain into the circulation. Proceedings of the National Academy of Sciences, USA, 93, 9799-9804.

Lutjohann, D., Papassotiropoulos, A., Bjorkhem, I., Locatelli, S., Bagli, M., Oehring, R. D. et al. (2000). Plasma 24S-hydroxycholesterol (cerebrosterol) is increased in Alzheimer and vascular disease patients. Journal of Lipid Research, 41, 195-198.

Mahley, R. W. \& Rall, Jr. S. C. (2000). Apolipoprotein E: far more than a lipid transport protein. Annual Review Genomics Human Genetics, 01, 507-537.

Maier, S. F. \& Watkins, L. R. (2003). Immune-to-central nervous system communication an its role in modulating pain and cognition: implications for cancer and cancer treatment. Brain.Behav.Immun., 17, S125-S131.

Manelli, A. M., Bulfinch, L. C., Sullivan, P. M., \& Ladu, M. J. (2007). Abeta42 neurotoxicity in primary co-cultures: effect of apoE isoform and Abeta conformation. Neurobiology of Aging, 28, 1139-1147.

Mantyh, P. W., Ghilardi, J. R., Rogers, S., Demaster, E., Allen, C. J., Stimson, E. R. et al. (1993). Aluminum, iron, and zinc ions promote aggregation of physiological concentrations of beta-amyloid peptide. Journal of Neurochemistry, 61, 1171-1174.

Marcus, J., Honigbaum, S., Shroff, S., Honke, K., Rosenbluth, J., \& Dupree, J. L. (2006). Sulfatide is essential for the maintenance of CNS myelin and axon structure. Glia, 53, $372-381$.

Martin, V., Fabelo, N., Santpere, G., Puig, B., Marin, R., Ferrer, I. et al. (2009). Lipid Alterations in Lipid Rafts from Alzheimer's Disease Human Brain Cortex. J.Alzheimers.Dis..

Martins, I. J., Berger, T., Sharman, M. J., Verdile, G., Fuller, S. J., \& Martins, R. N. (2009). Cholesterol metabolism and transport in the pathogenesis of Alzheimer's disease. Journal of Neurochemistry, 111, 1275-1308.

Masters, C. L., Simms, G., Weinman, N. A., Multhaup, G., McDonald, B. L., \& Beyreuther, K. (1985). Amyloid plaque core protein in Alzheimer disease and Down syndrome. Proc.Natl.Acad.Sci.U.S.A, 82, 4245-4249. 
Matthews, D. B., Morrow, A. L., O'Buckley, T., Flanigan, T. J., Berry, R. B., Cook, M. N. et al. (2008). Acute mild footshock alters ethanol drinking and plasma corticosterone levels in $\mathrm{C} 57 \mathrm{BL} / 6 \mathrm{~J}$ male mice, but not DBA/2J or A/J male mice. Alcohol, 42, 469-476.

Matthews, D. B. \& Silvers, J. R. (2004). The use of acute ethanol administration as a tool to investigate multiple memory systems. Neurobiology of Learning and Memory, 82, 299-308.

Mauch, D. H., Nagler, K., Schumacher, S., Goritz, C., Muller, E.-C., Otto, A. et al. (2001). CNS synaptogenesis promoted by glial-derived cholesterol. Science, 294, 13541357.

Meaney, S., Hassan, M., Sakinis, A., Lutjohann, D., von Bergmann, K., Wennmalm, A. et al. (2001). Evidence that the major oxysterols in human circulation originate from distinct pools of cholesterol: a stable isotope study. Journal of Lipid Research, 42, 70-78.

Mielke, M. M., Zandi, P. P., Sjogren, M., Gustafson, D., Ostling, S., Steen, B. et al. (2005). High total cholesterol levels in late life associated with a reduced risk of dementia. Neurology, 64, 1689-1695.

Miller, S. \& Wehner, J. M. (1994). Cholesterol treatment facilitates spatial learning performance in DBA/2Ibg mice. Pharmacology Biochemistry and Behavior, 49, 257-261.

Milward, E. A., Papadopoulos, R., Fuller, S. J., Moir, R. D., Small, D., Beyreuther, K. et al. (1992). The amyloid protein precursor of Alzheimer's disease is a mediator of the effects of nerve growth factor on neurite outgrowth. Neuron, 9, 129-137.

Mitter, D., Reisinger, C., Hinz, B., Hollmann, S., Yelamanchili, S. V., Trieber-Hels, S. et al. (2003). The synaptophysin/synaptobrevin interaction critically depends on the cholesterol content. Journal of Neurochemistry, 84, 35-42.

Mori, T., Paris, D., Town, T., Rojiani, A. M., Sparks, D. L., Delledonne, A. et al. (2001). Cholesterol accumulates in senile plaques of Alzheimer disease patients and in transgenic APP mice. Journal of Neuropathology and Experimental Neurology, 60, 778-785.

Morley, J. E., Farr, S. A., Banks, W. A., Johnson, S. N., Yamada, K. A., \& Xu, L. (2010). A physiological role for amyloid-beta protein:enhancement of learning and memory. J.Alzheimers.Dis., 19, 441-449. 
Moyer, J. R., Jr., Deyo, R. A., \& Disterhoft, J. F. (1990). Hippocampectomy disrupts trace eye-blink conditioning in rabbits. Behavioral Neuroscience, 104, 243-252.

Muaki, H., Tsurugizawa, T., Ogiue-Ikeda, M., Murakami, G., Hojo, Y., Ishii, H. et al. (2006). Local neurosteroid production in the hippocampus: influence on synaptic plasticity of memory. Neuroendocrinology, 84, 255-263.

Mulder, M., Blokland, A., van den Berg, D.-K., Schulten, H., Bakker, A. H. F., Terwel, D. et al. (2001). Apolipoprotein E protects against neuropathology induced by a high-fat diet and maintains the integrity of the blood-brain barrier during aging. Laboratory Investigation, 81, 953-960.

Muldoon, M. F., Ryan, C. M., Matthews, K. A., \& Manuck, S. B. (1997). Serum cholesterol and intellectual performance. Psychosomatic Medicine, 59, 382-387.

Näslund, J., Haroutunian, V., Mohs, R., Davis, K. L., Davies, P., Greengard, P. et al. (2000). Correlation between elevated levels of amyloid $\beta$-peptide in the brain and cognitive decline. JAMA, 283, 1571-1577.

Nayler, W. G. (1999). Review of preclinical data of calcium channel blockers and atherosclerosis. Journal of Cardiovascular Pharmacology, 33, S7-S-11.

Nelson, T. J., Schreurs, B. G., \& Alkon, D. L. (1999). Memory storage mechanisms: GTP-binding proteins and conservation across species. In G.Adelman \& B. H. Smith (Eds.), Elsevier's Encyclopedia of Neuroscience (2nd ed., pp. 1138-1141). New York: Elsevier.

Nimmrich, V., Grimm, C., Draguhn, A., Barghorn, S., Lehmann, A., Schoemaker, H. et al. (2008). Amyloid beta oligomers (A beta(1-42) globulomer) suppress spontaneous synaptic activity by inhibition of P/Q-type calcium currents. Journal of Neuroscience, 28, 788-797.

Norton WT \& Cammer W. (1984). Isolation and characterization of myelin. [Morell P (ed)], 147-195. New York, NY, Plenum.

Ref Type: Generic

Notkola, I.-L., Sulkava, R., Pekkanen, J., Erkinjunti, T., Ehnholm, C., Kivinen, P. et al. (1998). Serum total cholesterol, apolipoprotein E $\varepsilon 4$ allele, and Alzheimer's disease. Neuroepidemiology, 17, 14-20. 
O'Brien, W. T., Xu, G., Batta, A., Tint, G. S., Salen, G., Dyer, C. A. et al. (2002). Developmental sensitivity of associative learning to cholesterol synthesis inhibitors. Behavioural Brain Research, 129, 141-152.

O'Brien, W. T., Xu, G., Tint, G. S., Salen, G., \& Servatius, R. J. (2000). Blocking cholesterol synthesis impairs acquisition of the classically conditioned eyeblink response. Integrative Physiological and Behavioral Science, 35, 120-131.

Olariu, A., Cleaver, K. M., Shore, L. E., Brewer, M. D., \& Cameron, H. A. (2005). A natural form of learning can increase and decrease the survival of new neurons in the dentate gyrus. Hippocampus, 15, 750-762.

Panza, F., D'Introno, A., Colacicco, A. M., Capurso, C., Pichichero, G., Capurso, S. A. et al. (2006). Lipid metabolism in cognitive decline and dementia. Brain Research Reviews, $51,275-292$.

Papassotiropoulos, A., Lutjohann, D., Bagli, M., Locatelli, S., Jessen, F., Buschfort, R. et al. (2002). 24S-hydroxycholesterol in cerebrospinal fluid is elevated in early stages of dementia. Journal of Psychiatric Research, 36, 27-32.

Papassotiropoulos, A., Wollmer, M. A., Tsolaki, M., Brunner, F., Molyva, D., Lutjohann, D. et al. (2005). A cluster of cholesterol-related genes confers susceptibility for Alzheimer's disease. Journal of Clinical Psychiatry, 66, 940-947.

Parodi, J., Sep+'Iveda, F. J., Roa, J., Opazo, C., Inestrosa, N. C., \& Aguayo, L. G. (2010). +,-Amyloid Causes Depletion of Synaptic Vesicles Leading to Neurotransmission Failure. Journal of Biological Chemistry, 285, 2506-2514.

Pearson, H. A. \& Peers, C. (2006). Physiological roles for amyloid $\beta$ peptides. Journal of Physiology, 575, 5-10.

Pernber, Z., Molander-Melin, M., Berthold, C. H., Hansson, E., \& Fredman, P. (2002). Expression of the myelin and oligodendrocyte progenitor marker sulfatide in neurons and astrocytes of adult rat brain. J.Neurosci.Res., 69, 86-93.

Pfrieger, F. W. (2002). Role of glia in synapse development. Current Opinion in Neurobiology, 12, 486-490. 
Pfrieger, F. W. (2003a). Cholesterol homeostasis and function in neurons of the central nervous system. Cellular and Molecular Life Sciences, 60, 1158-1171.

Pfrieger, F. W. (2003b). Role of cholesterol in synapse formation and function. Biochimica et Biophysica Acta, 1610, 271-280.

Pham, K., McEwen, B. S., Ledoux, J. E., \& Nader, K. (2005). Fear learning transiently impairs hippocampal cell proliferation. Neuroscience, 130, 17-24.

Plant, L. D., Boyle, J. P., Smith, I. F., Peers, C., \& Pearson, H. A. (2003). The production of amyloid $\beta$ peptide is a critical requirement for the viability of central neurons. Journal of Neuroscience, 23, 5531-5535.

Plant, L. D., Webster, N. J., Boyle, J. P., Ramsden, M., Frier, D. B., Peers, C. et al. (2006). Amyloid $\beta$ peptide as a physiological modulator of neuronal "A"-type $\mathrm{K}^{+}$current. Neurobiology of Aging, 27, 1673-1683.

Poirier, J. (2005). Apolipoportein E, cholesterol transport and synthesis in sporadic Alzheimer's disease. Neurobiol.Aging, 26, 355-361.

Port, R. L., Romano, A. G., Steinmetz, J. E., Mikhail, A. A., \& Patterson, M. M. (1986). Retention and acquisition of classical trace conditioned responses by rabbits with hippocampal lesions. Behavioral Neuroscience, 100, 745-752.

Puglielli, L., Tanzi, R. E., \& Kovacs, D. M. (2003). Alzheimer's disease: the cholesterol connection. Nature Neuroscience, 6, 345-351.

Qiu, S., Korwek, K. M., \& Weeber, E. J. (2005). A fresh look at an ancient family: emerging roles for low density lipoprotein receptors in synaptic plasticity and memory formation. Neurobiology of Learning and Memory, 85, 16-29.

Quartermain, D. (2000). Chronic administration of the $\mathrm{Ca}^{2+}$ channel blocker amlodipine facilitates learning and memory in mice. European Journal of Pharmacology, 399, 57-63.

Rahman, S. M. A., Van Dam, A.-M., Schultzberg, M., \& Crisby, M. (2005). High cholesterol diet results in increased expression of interleukin- 6 and caspase- 1 in the brain of apolipoprotein E knockout and wild type mice. Journal of Neuroimmunology, 169, 5967. 
Ramakrishnan, H., Hedayati, K. K., Lullmann-Rauch, R., Wessig, C., Fewou, S. N., Maier, H. et al. (2007). Increasing sulfatide synthesis in myelin-forming cells of arylsulfatase A-deficient mice causes demyelination and neurological symptoms reminiscent of human metachromatic leukodystrophy. Journal of Neuroscience, 27, 9482-9490.

Ramsden, M., Plant, L. D., Webster, N. J., Vaughan, P. F. T., Henderson, Z., \& Pearson, H. A. (2001). Differential effects of unaggregated and aggregated amyloid $\beta$ protein (140) on $\mathrm{K}^{+}$channel currents in primary cultures of rat cerebellar granule and cortical neurones. Journal of Neurochemistry, 79, 699-712.

Reitan, R. M. \& Shipley, R. E. (1963). The relationship of serum cholesterol changes to psychological abilities. Journal of Gerontology, 18, 350-357.

Reitz, C., Luchsinger, J. A., Tang, M.-X., Manly, J., \& Mayeux, R. (2005). Impact of plasma lipids and time on memory performance in healthy elderly without dementia. Neurology, 64, 1378-1383.

Roberts, G. W., Gentleman, S. M., Lynch, A., \& Graham, D. I. (1991). beta A4 amyloid protein deposition in brain after head trauma. Lancet, 338, 1422-1423.

Rockwood, K. (2006). Epidemiological and clinical trials evidence about a preventive role for statins in ALzheimer's disease. Acta Neurologica Scandanavia, 114, s71-s77.

Rockwood, K., Kirkland, S., Hogan, D. B., McKnight, C., Merry, H., Verreault, R. et al. (2002). Use of lipid-lowering agents, indication bias, and the risk of dementia in community-dwelling elderly people. Archives of Neurology, 59, 223-227.

Rottkamp, C. A., Raina, A. K., Zhu, X., Gaier, E., Bush, A. I., Atwood, C. S. et al. (2001). Redox-active iron mediates amyloid-beta toxicity. Free Radic.Biol.Med., 30, 447450.

Runyan, J. D., Moore, A. N., \& Dash, P. K. (2004). A Role for Prefrontal Cortex in Memory Storage for Trace Fear Conditioning. Journal of Neuroscience, 24, 1288-1295.

Sadovnick, A. D., Tuokko, H., Applegarth, D. A., Toone, J. R., Hadjistavropoulos, T., \& Beattie, B. L. (1993). The differential diagnosis of adult onset metachromatic leukodystrophy and early onset familial Alzheimer disease in an Alzheimer clinic population. Can.J.Neurol.Sci., 20, 312-318. 
Saher, G., Brugger, B., Lappe-Siefke, C., Mobius, W., Tozawa, R.-I., Wehr, M. et al. (2005). High cholesterol level is essential for myelin membrane growth. Nature Neuroscience, 8, 468-475.

Salminen, A., Ojala, J., Suuronen, T., Kaarniranta, K., \& Kauppinen, A. (2008). Amyloid-beta oligomers set fire to inflammasomes and induce Alzheimer's pathology. J.Cell Mol.Med., 12, 2255-2262.

Schachter, M. (1997). Calcium antagonists and atherosclerosis. International Journal of Cardiology, 62, S9-S15.

Schaeren-Wiemers, N., van der, B. P., \& Schwab, M. E. (1995). The UDPgalactose:ceramide galactosyltransferase: expression pattern in oligodendrocytes and Schwann cells during myelination and substrate preference for hydroxyceramide. Journal of Neurochemistry, 65, 2267-2278.

Schneiderman, N., Fuentes, I., \& Gormezano, I. (1962). Acquisition and extinction of the classically conditioned eyelid response in the albino rabbit. Science, 136, 650-652.

Schoknecht, P. A., Ebner, S., Pond, W. G., Zhang, S., McWhinney, V., Wong, W. W. et al. (1994). Dietary cholesterol supplementation improves growth and behavioral response of pigs selected for genetically high and low serum cholesterol. J.Nutr., 124, 305-314.

Schonknecht, P., Lutjohann, D., Pantel, J., Bardenheuer, H., Hartmann, T., von Bergmann, K. et al. (2002). Cerebrospinal fluid 24S-hydroxycholesterol is increased in patients with Alzheimer's disease compared to healthy controls. Neuroscience Letters, $324,83-85$.

Schrader, L. A., Anderson, A. E., Varga, A. W., Levy, M., \& Sweatt, J. D. (2002). The other half of Hebb: $\mathrm{K}^{+}$channels and the regulation of neuronal excitability in the hippocampus. Molecular Neurobiology, 25, 51-66.

Schreurs, B. G. (1993). Long-term memory and extinction of the classically conditioned rabbit nictitating membrane response. Learning \& Motivation, 24, 293-302.

Schreurs, B. G. (1998). Long-term memory and extinction of rabbit nictitating membrane trace conditioning. Learning \& Motivation, 29, 68-82. 
Schreurs, B. G. \& Alkon, D. L. (1990). US-US conditioning of the rabbit's nictitating membrane response: emergence of a conditioned response without alpha conditioning. Psychobiology, 18, 312-320.

Schreurs, B. G., Gusev, P. A., Tomsic, D., Alkon, D. L., \& Shi, T. (1998). Intracellular correlates of acquisition and long-term memory of classical conditioning in Purkinje cell dendrites in slices of rabbit cerebellar lobule HVI. Journal of Neuroscience, 18, 54985507.

Schreurs, B. G., Shi, T., Pineda, S. I., \& Buck, D. L. (2000). Conditioning the unconditioned response: modification of the rabbit's (Oryctolagus cuniculus) unconditioned nictitating membrane response. Journal of Experimental Psychology: Animal Behavior Processes, 26, 144-156.

Schreurs, B. G., Smith-Bell, C. A., Darwish, D. S., Stankovic, G., \& Sparks, D. L. (2007a). Classical conditioning of the rabbit's nictitating membrane response is a function of the duration of dietary cholesterol. Nutritional Neuroscience, 10, 159-168.

Schreurs, B. G., Smith-Bell, C. A., Darwish, D. S., Stankovic, G., \& Sparks, D. L. (2007b). High dietary cholesterol facilitates classical conditioning of the rabbit's nictitating membrane response. Nutritional Neuroscience, 10, 31-43.

Schreurs, B. G., Smith-Bell, C. A., Darwish, D. S., Wang, D., Burhans, L., GonzalesJoekes, J. et al. (2007c). Cholesterol enhances classical conditioning of the rabbit heart rate response. Behavioural Brain Research, 181, 52-63.

Schreurs, B. G., Smith-Bell, C. A., Lochhead, J., \& Sparks, D. L. (2003). Cholesterol modifies classical conditioning of the rabbit (Oryctolagus cuniculus) nictitating membrane response. Behavioral Neuroscience, 117, 1220-1232.

Servatius, R. J. \& Beck, K. D. (2003). Facilitated acquisition of the classically conditioned eyeblink response in male rats after systemic IL-1 $\beta$. Integrative Physiological and Behavioral Science, 38, 169-178.

Seubert, P., Oltersdorf, T., Lee, M. G., Barbour, R., Blomquist, C., Davis, D. L. et al. (1993). Secretion of beta-amyloid precursor protein cleaved at the amino terminus of the beta-amyloid peptide. Nature, 361, 260-263. 
Shapiro, E. G., Lockman, L. A., Balthazor, M., \& Krivit, W. (1995). Neuropsychological outcomes of several storage diseases with and without bone marrow transplantation.

J.Inherit.Metab Dis., 18, 413-429.

Shapiro, E. G., Lockman, L. A., Knopman, D., \& Krivit, W. (1994). Characteristics of the dementia in late-onset metachromatic leukodystrophy. Neurology, 44, 662-665.

Shobab, L. A., Hsiung, G.-Y., \& Feldman, H. H. (2005). Cholesterol in Alzheimer's disease. Lancet Neurology, 4, 841-852.

Simakova, O. \& Arispe, N. J. (2006). Early and late cytotoxic effects of external application of the Alzheimer's Abeta result from the initial formation and function of Abeta ion channels. Biochemistry, 45, 5907-5915.

Simons, A., Ruppert, T., Schmidt, C., Schlicksupp, A., Pipkorn, R., Reed, J. et al. (2002a). Evidence for a copper-binding superfamily of the amyloid precursor protein. Biochemistry, 41, 9310-9320.

Simons, M., Keller, P., Dichgans, J., \& Schulz, J. B. (2001). Cholesterol and Alzheimer's disease: Is there a link? Neurology, 57, 1089-1093.

Simons, M., Schwarzler, F., Lutjohann, D., von Bergmann, K., Beyreuther, K., Dichgans, J. et al. (2002b). Treatment with simvastatin in normocholesterolemic patients with Alzheimer's disease: a 26-week randomized, placebo-controlled, double-blind trial. Annals of Neurology, 52, 346-350.

Sjogren, M., Gustafsson, K., Syversen, S., Olsson, A., Edman, A., Davidsson, P. et al. (2003). Treatment with simvastatin in patients with Alzheimer's disease lowers both $\alpha$ and $\beta$-cleaved amyloid precursor protein. Dementia and Geriatric Cognitive Disorders, $16,25-30$.

Sjogren, M., Mielke, M., Gustafson, D., Zandi, P., \& Skoog, I. (2006). Cholesterol and Alzheimer's disease - is there a relation? Mechanisms of Ageing and Development, 127, 138-147.

Snyder, J. S., Hong, N. S., McDonald, R. J., \& Wojtowicz, J. M. (2005). A role for adult neurogenesis in spatial long-term memory. Neuroscience, 130, 843-852. 
Solomon, A., Kareholt, I., Ngandu, T., Winblad, B., Nissinen, A., Tuomilehto, J. et al. (2007). Serum cholesterol changes after midlife and late-life cognition. Neurology, 68, 751-756.

Solomon, A., Kivipelto, M., Wolozin, B., Zhou, J., \& Whitmer, R. A. (2009). Midlife serum cholesterol and increased risk of Alzheimer's and vascular dementia three decades later. Dementia and Geriatric Cognitive Disorders, 28, 75-80.

Sooksawate, T. \& Simmonds, M. A. (2001a). Effects of membrane cholesterol on the sensitivity of the $\mathrm{GABA}_{\mathrm{A}}$ receptor to $\mathrm{GABA}$ in acutely dissociated rat hippocampal neurones. Neuropharmacology, 40, 178-184.

Sooksawate, T. \& Simmonds, M. A. (2001b). Influence of membrane cholesterol on modulation of the $\mathrm{GABA}_{\mathrm{A}}$ receptor by neuroactive steroids and other potentiators. British Journal of Pharmacology, 134, 1303-1311.

Spady, D. K. \& Dietschy, J. M. (1983). Sterol synthesis in vivo in 18 tissues of the squirrel monkey, guinea pig, hamster, and rat. Journal of Lipid Research, 24, 303-315.

Spain, J. W. \& Newsom, G. C. (1991). Chronic opioids impair acquisition of both radial maze and Y-maze choice escape. Psychopharmacology (Berl), 105, 101-106.

Sparks, D. L. (1996). Intraneuronal $\beta$-amyloid immunoreactivity in the CNS. Neurobiology of Aging, 17, 291-299.

Sparks, D. L. (1997a). Coronary artery disease, hypertension, ApoE and cholesterol: a link to Alzheimer's disease. Annals New York Academy of Sciences, 826, 128-146.

Sparks, D. L. (1997b). Dietary cholesterol induces Alzheimer-like $\beta$-amyloid immunoreactivity in rabbit brain. Nutrition, Metabolism and Cardiovascular Diseases, 7, 255-266.

Sparks, D. L., Connor, D. J., Sabbagh, M. N., Petersen, R. B., Lopez, J., \& Browne, P. (2006a). Circulating cholesterol levels, apolipoprotein E genotype and dementia severity influence the benefit of atorvastatin treatment in Alzheimer's disease: results of the Alzheimer's Disease Cholesterol-Lowering Treatment (ADCLT) trial. Acta Neurologica Scandinavica, 114, s3-s7. 
Sparks, D. L., Friedland, R., Petanceska, S., Schreurs, B. G., Shi, J., Perry, G. et al. (2006b). Trace copper levels in the drinking water, but not zinc or aluminum influence CNS Alzheimer-like pathology. Journal of Nutrition, Health and Aging, 10, 247-254.

Sparks, D. L., Hunsaker III, J. C., Scheff, S. W., Kryscio, R. J., Henson, J. L., \& Markesbery, W. R. (1990). Cortical senile plaques in coronary heart disease, aging and Alzheimer's disease. Neurobiology of Aging, 11, 601-607.

Sparks, D. L., Kuo, Y.-M., Roher, A. E., Martin, T. A., \& Lukas, R. J. (2000a). Alterations of Alzheimer's disease in the cholesterol-fed rabbit, including vascular inflammation. Preliminary observations. Annals New York Academy of Sciences, 903, $335-344$.

Sparks, D. L., Liu, H., Gross, D. R., \& Scheff, S. W. (1995). Increased density of cortical apolipoprotein $\mathrm{E}$ immunoreactive neurons in rabbit brain after dietary administration of cholesterol. Neuroscience Letters, 187, 142-144.

Sparks, D. L., Lochhead, J., Horstman, D., Wagoner, T., \& Martin, T. (2002). Water quality has a pronounced effect on cholesterol-induced accumulation of Alzheimer amyloid $(\mathrm{a} \beta)$ in rabbit brain. Journal of Alzheimer's Disease, 4, 523-529.

Sparks, D. L., Martin, T. A., Gross, D. R., \& Hunsaker III, J. C. (2000b). Link between heart disease, cholesterol and Alzheimer's disease: a review. Microscopy Research and Technique, 50, 287-290.

Sparks, D. L., Scheff, S. W., Hunsaker III, J. C., Liu, H., Landers, T., \& Gross, D. R. (1994). Induction of Alzheimer-like $\beta$-amyloid immunoreactivity in the brains of rabbits with dietary cholesterol. Experimental Neurology, 126, 88-94.

Sparks, D. L. \& Schreurs, B. G. (2003). Trace amounts of copper in water induce $\beta$ amyloid plaques and learning deficits in a rabbit model of Alzheimer's disease. Proceedings of the National Academy of Sciences, USA, 100, 11065-11069.

Stewart, R., Russ, C., Richards, M., Brayne, C., Lovestone, S., \& Mann, A. (2001). Apolipoprotein E genotype, vascular risk and early cognitive impairment in an African Caribbean population. Dementia and Geriatric Cognitive Disorders, 12, 251-256.

Taddei, K., Laws, S. M., Verdile, G., Munns, S., D'Costa, K., Harvey, A. R. et al. (2010). Novel phage peptides attenuate beta amyloid-42 catalysed hydrogen peroxide production and associated neurotoxicity. Neurobiology of Aging, 31, 203-214. 
Tanapat, P., Hastings, N. B., Reeves, A. J., \& Gould, E. (1999). Estrogen stimulates a transient increase in the number of new neurons in the dentate gyrus of the adult female rat. Journal of Neuroscience, 19, 5792-5801.

Teunissen, C. E., de Vente, J., Steinbusch, H. W. M., \& De Bruijn, C. (2002).

Biochemical markers related to Alzheimer's dementia in serum and cerebrospinal fluid. Neurobiology of Aging, 23, 485-508.

Teunissen, C. E., de Vente, J., von Bergmann, K., Bosma, H., Van Boxtel, M. P. J., De Bruijn, C. et al. (2003). Serum cholesterol, precursors and metabolites and cognitive performance in an aging population. Neurobiology of Aging, 24, 147-155.

Thirumangalakudi, L., Prakasam, A., Zhang, R., Bimonte-Nelson, H. A., Sambamurti, K., Kindy, M. S. et al. (2008). High cholesterol-induced neuroinflammation and amyloid precursor protein processing correlate with the loss of working memory in mice. Journal of Neurochemistry, 106, 475-485.

Thompson, R. F. \& Kim, J. J. (1996). Memory systems in the brain and localization of a memory. Proceedings of the National Academy of Sciences, USA, 93, 13438-13444.

Thomson, L. M. \& Sutherland, R. J. (2005). Systemic administration of lipopolysaccharide and interleukin- $1 \beta$ have different effects on memory consolidation. Brain Research Bulletin, 67, 24-29.

Thudichum, J. (1884). A treatise on the chemical constitution of the brain. London, Balliere, Tindall, and Cox.

Ref Type: Generic

Tuma, P. L. \& Hubbard, A. L. (2003). Transcytosis: crossing cellular barriers. Physiological Reviews, 83, 871-932.

Turchin, A., Wiebe, D. A., Seely, E. W., Graham, T., Longo, W., \& Soiffer, R. (2004). Severe hypercholesterolemia mediated by lipoprotein $\mathrm{X}$ in patients with chronic graftversus-host disease of the liver. Bone Marrow Transplant, 35, 85-89.

Upchurch, M. \& Wehner, J. M. (1988). DBA/2Ibg mice are incapable of cholinergicallybased learning in the Morris water maze. Pharmacology Biochemistry \& Behavior, 29, 325-329. 
van den Kommer, T. N., Dik, M. G., Comijs, H. C., Fassbender, K., Lutjohann, D., \& Jonker, C. (2009). Total cholesterol and oxysterols: early markers for cognitive decline in elderly? Neurobiology of Aging, 30, 534-545.

Van Der, B. K., Meerlo, P., Luiten, P. G., Eggen, B. J., \& Van der Zee, E. A. (2005). Effects of active shock avoidance learning on hippocampal neurogenesis and plasma levels of corticosterone. Behav.Brain Res., 157, 23-30.

van Exel, E., de Craen, A. J. M., Gussekloo, J., Houx, P., Bootsma-van der Weil, A., Macfarlane, P. W. et al. (2002). Association between high-density lipoprotein and cognitive impairment in the oldest old. Annals of Neurology, 51, 716-721.

van, P. H., Kempermann, G., \& Gage, F. H. (1999). Running increases cell proliferation and neurogenesis in the adult mouse dentate gyrus. Nat.Neurosci., 2, 266-270.

varez-Buylla, A., Kirn, J. R., \& Nottebohm, F. (1990). Birth of projection neurons in adult avian brain may be related to perceptual or motor learning. Science, 249, 14441446.

Vassar, R. (2005). beta-Secretase, APP and Abeta in Alzheimer's disease.

Subcell.Biochem., 38, 79-103.

Vassar, R., Bennett, B. D., Babu-Khan, S., Kahn, S., Mendiaz, E. A., Denis, P. et al. (1999). Beta-secretase cleavage of Alzheimer's amyloid precursor protein by the transmembrane aspartic protease BACE. Science, 286, 735-741.

Vauthey, C., de Freitas, G. R., van Melle, G., Devuyst, G., \& Bogousslavsky, J. (2000). Better outcome after stroke with higher serum cholesterol levels. Neurology, 54, 19441949.

Vega, G. L., Weiner, M. F., Lipton, A. M., von Bergmann, K., Lutjohann, D., Moore, C. et al. (2003). Reduction in levels of 24S-hydroxycholesterol by statin treatment in patients with Alzheimer disease. Archives of Neurology, 60, 510-515.

Voikar, V., Rauvala, H., \& Ikonen, E. (2002). Cognitive deficit and development of motor impairment in a mouse model of Niemann-Pick type $\mathrm{C}$ disease. Behavioural Brain Research, 132, 1-10. 
Waddell, J. \& Shors, T. J. (2008). Neurogenesis, learning and associative strength. European Journal of Neuroscience, 27, 3020-3028.

Walsh, D. M., Klyubin, I., Fadeeva, J. V., Cullen, W. K., Anwyl, R., Wolfe, M. S. et al. (2002). Naturally secreted oliogomers of amyloid $\beta$ protein potently inhibit hippocampal long-term potentiation in vivo. Nature, 416, 535-539.

Wasser, C. R., Ertunc, M., Liu, X., \& Kavalali, E. T. (2007). Cholesterol-dependent balance between evoked and spontaeous synaptic vesicle recycling. J.Physiol.(Lond)., 579, 413-429.

Weible, A. P., McEchron, M. D., \& Disterhoft, J. F. (2000). Cortical involvement in acquisition and extinction of trace eyeblink conditioning. Behavioral Neuroscience, 114, 1058-1067.

Weiss, C., Bouwmeester, H., Power, J. M., \& Disterhoft, J. F. (1999). Hippocampal lesions prevent trace eyeblink conditioning in the freely moving rat. Behavioural Brain Research, 99, 123-132.

West, R., Beeri, M. S., Schmeidler, J., Hannigan, C. M., Angelo, G., Grossman, H. T. et al. (2008). Better memory functioning associated with higher total and low-density lipoprotein cholesterol levels in very elderly subjects without the apolipoprotein e4 allele. American Journal of Geriatric Psychiatry, 16, 781-785.

Whitmer, R. A., Sidney, S., Selby, J., Johnston, S. C., \& Yaffe, K. (2005). Midlife cardiovascular risk factors and risk of dementia in late life. Neurology, 64, 277-281.

Whitney, K. D., Watson, M. A., Collins, J. L., Benson, W. G., Stone, T. M., Numerick, M. J. et al. (2002). Regulation of cholesterol homeostasis by the liver X receptors in the central nervous system. Molecular Endocrinology, 16, 1378-1385.

Wolozin, B., Kellman, W., Celesia, G. C., \& Siegel, G. (2000). Decreased prevalence of Alzheimer disease associated with 3-hydroxy-3-methyglutaryl coenzyme A reductase inhibitors. Archives of Neurology, 57, 1439-1443.

Wood, W. G., Igbavboa, U., Exkert, G. P., Johnson-Anuna, L. N., \& Muller, W. E. (2005). Is hypercholesterolemia a risk factor for Alzheimer's disease. Molecular Neurobiology, 31, 185-192. 
Woodruff-Pak, D. S., Agelan, A., \& Del Valle, L. (2007). A rabbit model of Alzheimer's disease: valid at neuropathological, cognitive, and therapeutic levels. Journal of Alzheimer's Disease, 11, 371-383.

Woodruff-Pak, D. S., Chi, J., Li, Y.-T., Pak, M. H., \& Fanelli, R. J. (1997). Nimodipine ameliorates impaired eyeblink classical conditioning in order rabbits in the long-delay paradigm. Neurobiology of Aging, 18, 641-649.

Xi, L., Ghosh, S., Wang, X., Das, A., Anderson, F. P., \& Kukreja, R. C. (2006). Hypercholestrolemia enhances tolerance to lethal systemic hypoxia in middle-aged mice: possible role of VEGF downregulation in brain. Mol.Cell.Biochem., 291, 205-211.

Xie, C., Burns, D. K., Turley, S. D., \& Dietschy, J. M. (2000). Cholesterol is sequestered in the brains of mice with Niemann-Pick C disease but turnover is increased. Journal of Neuropathology and Experimental Neurology, 59, 1106-1117.

Xie, C., Lund, E. G., Turley, S. D., Russell, D. W., \& Dietschy, J. M. (2003).

Quantitation of two pathways for cholesterol excretion from the brain in normal mice and mice with neurodegeneration. Journal of Lipid Research, 44, 1780-1789.

Xu, G., Servatius, R. J., Shefer, S., Tint, G. S., O'Brien, W. T., Batta, A. K. et al. (1998). Relationship between abnormal cholesterol synthesis and retarded learning in rats. Metabolism, 47, 878-882.

$\mathrm{Xu}$, J. \& Kang, J. (2005). The mechanisms and functions of activity-dependent long-term potentiation of intrinsic excitability. Reviews in the Neurosciences, 16, 311-323.

Yaffe, K., Barret-Connor, E., Lin, F., \& Grady, D. (2002). Serum lipoprotein levels, statin use, and cognitive function in older women. Archives of Neurology, 59, 378-384.

Yanagisawa, K. (2003). Cholesterol and A $\beta$ aggregation. Pharmacopsychiatry, 36, S127S129.

Yang, D. S., McLaurin, J., Qin, K., Westaway, D., \& Fraser, P. E. (2000). Examining the zinc binding site of the amyloid-beta peptide. Eur.J.Biochem., 267, 6692-6698.

Yankner, B. A., Dawes, L. R., Fisher, S., Villa-Komaroff, L., Oster-Granite, M. L., \& Neve, R. L. (1989). Neurotoxicity of a fragment of the amyloid precursor associated with Alzheimer's disease. Science, 245, 417-420. 
Yankner, B. A., Duffy, L. K., \& Kirschner, D. A. (1990). Neurotrophic and neurotoxic effects of amyloid beta protein: reversal by tachykinin neuropeptides. Science, 250, 279282.

Yehuda, S. \& Carasso, R. L. (1993). Modulation of learning, pain thresholds, and thermoregulation in the rat by preparations of free purified $\alpha$-linolenic and linoleic acids: determination of the optimal $\omega 3$-to- $\omega 6$ ratio. Proceedings of the National Academy of Sciences, USA, 90, 10345-10349.

Yehuda, S., Rabinovitz, S., \& Mostofsky, D. I. (2005). Mediation of cognitive function by high fat diet following stress and inflammation. Nutritional Neuroscience, 8, 309-315.

Yehuda, S., Rabinovitz, S., \& Motofsky, D. I. (1998). Modulation of learning and neuronal membrane composition in the rat by essential fatty acid preparation: time-course analysis. Neurochemical Research, 23, 627-634.

Yin, X., Baek, R. C., Kirschner, D. A., Peterson, A., Fujii, Y., Nave, K. A. et al. (2006). Evolution of a neuroprotective function of central nervous system myelin. J.Cell Biol., 172, 469-478.

Zandi, P. P., Sparks, D. L., Khachaturian, A. S., Tschanz, J. T., Norton, M., Steinberg, M. et al. (2005). Do statins reduce risk of incident dementia and Alzheimer's disease? Archives of General Psychiatry, 62, 217-224.

Zeng, Y. \& Han, X. (2008). Sulfatides facilitate apolipoprotein E-mediated amyloid-beta peptide clearance through an endocytotic pathway. Journal of Neurochemistry, 106, 1275-1286.

Zhang, W. \& Linden, D. J. (2003). The other side of the engram: experience-driven changes in neuronal intrinsic excitability. Nature Reviews Neuroscience, 4, 885-900.

Zlokovic, B. V. (2005). Neurovascular mechanisms of Alzheimer's neurodegeneration. Trends in Neurosciences, 28, 202-208.

Zou, K., Gong, J. S., Yanagisawa, K., \& Michikawa, M. (2002). A novel function of monomeric amyloid beta-protein serving as an antioxidant molecule against metalinduced oxidative damage. Journal of Neuroscience, 22, 4833-4841. 
Zuliani, G., Cherubini, A., Atti, A. R., Ble, A., Vavalle, C., Di Todaro, F. et al. (2004). Low cholesterol levels are associated with short-term mortality in older patients with ischemic stroke. Journal of Gerentology: Medical Sciences, 59A, 293-297. 


\section{CIRRICULUM VITAE}

\section{DEYA S. DARWISH, B. A.}

\section{PERSONAL}

Date of Birth:

Birthplace:

Address:
September 17, 1978

Fairfax, VA

Department of Neurobiology and Anatomy WVU School of Medicine Robert C. Byrd Health Sciences Center PO Box 9302

Morgantown WV 26506

Work: (304) 293-0496

E-mail: ddarwish@hsc.wvu.edu

\section{EDUCATION}

High School:

Sherando High School

Stephen City, Virginia

College:

Shepherd University

Shepherdstown, West Virginia

B. S. Chemistry 2002

Suma cum Laude

Graduate:

WVU School of Medicine

Morgantown, West Virginia

PhD- Defended 6/07/10

"Dietary Cholesterol and Copper Affects on Learning, Memory and $A \beta$ : Learning and Memory Affects on Brain Cholesterol and Sulfatide Levels." 


\section{PUBLICATIONS}

\section{Articles}

1. Darwish, D.S., Wang, D., Konat, G., Schreurs, B. G. (2010). Dietary cholesterol impairs memory and memory increases brain cholesterol and sulfatide levels.

Behav.Neurosci., 124, 115-123.

2. Wang, D., Darwish, D. S., Schreurs, B. G., \& Alkon, D. L. (2008). Analysis of long-term cognitive-enhancing effects of bryostatin-1 on the rabbit (Oryctolagus cuniculus) nictitating membrane response. Behav.Pharmacol., 19, 245-256.

3. Schreurs, B. G., Smith-Bell, C. A., Darwish, D. S., Stankovic, G., \& Sparks, D. L. (2007). Classical conditioning of the rabbit's nictitating membrane response is a function of the duration of dietary cholesterol. Nutr.Neurosci., 10, 159-168.

4. Schreurs, B. G., Smith-Bell, C. A., Darwish, D. S., Stankovic, G., \& Sparks, D. L. (2007). High dietary cholesterol facilitates classical conditioning of the rabbit's nictitating membrane response. Nutr.Neurosci., 10, 31-43.

5. Schreurs, B. G., Smith-Bell, C. A., Darwish, D. S., Wang, D., Burhans, L. B., Gonzales-Joekes, J. et al. (2007). Cholesterol enhances classical conditioning of the rabbit heart rate response. Behav.Brain Res., 181, 52-63.

6. Wang, D., Darwish, D. S., \& Schreurs, B. G. (2006). Effects of 4-aminopyridine on classical conditioning of the rabbit (Oryctolagus cuniculus) nictitating membrane response. Behav.Pharmacol., 17, 319-329.

\section{PRESENTATIONS}

1. "Dietary Cholesterol and Copper Affects on Learning, Memory and A and Memory Affects on Brain Cholesterol and Sulfatide Levels." Thesis Defense, WVU Scholl of Medicine and Center for Neuroscience. June 2010.

2. "Dietary Cholesterol Impairs Memory and Memory Increases Brain Cholesterol and Sulfatide Levels." Van Liere Research Day, WVU-School of Medicine. Apr 2009.

3. "Dietary Cholesterol Impairs Memory and Memory Increases Brain Cholesterol and Sulfatide Levels." Neuroscience Retreat, WVU-School of Medicine. October 2009. 
4. "Dietary Cholesterol Impairs Memory and Memory Increases Brain Cholesterol and Sulfatide Levels." Neuroscience Retreat, WVU-School of Medicine. October 2008.

5. "Analysis of Long-Term Cognitive-Enhancing Effects of Bryostatin-1 on the Rabbit (Oryctolagus cuniculus) Nictitating Membrane Response." Van Liere Research Day, WVU-School of Medicine. Apr 2008.

\section{REFERENCES}

Bernard G. Schreurs, PhD

Professor

Department of Neurobiology and Anatomy

Blanchette Rockefeller Neurosciences Institute

West Virginia University School of Medicine

5700 HSS, PO Box 9302

Medical Center Drive

Morgantown WV 26506-9302

Work: (304) 293-0497

E-Mail: bschreurs@hsc.wvu.edu

Gregory W. Konat, PhD

Professor

Department of Neurobiology and Anatomy

Blanchette Rockefeller Neurosciences Institute

West Virginia University School of Medicine

5700 HSS, PO Box 9302

Medical Center Drive

Morgantown WV 26506-9302

Work: (304) 293-0497

E-Mail: gkonat@hsc.wvu.edu 